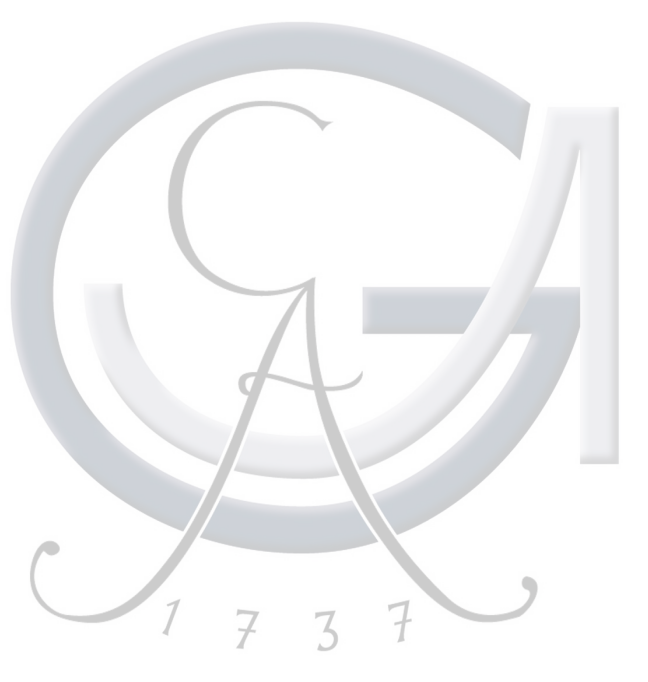

Mechanoelectrical Coupling and REORGANIZATION OF

Cardiomyocytes and Fibroblasts

UNDER SHEAR STRESS

Laura Turco 



\section{Mechanoelectrical Coupling and Reorganization of Cardiomyocytes and Fibroblasts Under Shear Stress}

\section{Dissertation}

to acquire the doctoral degree in mathematics and natural science

"Doctor rerum naturalium"

at the Georg-August-Universität Göttingen

within the doctoral degree program

Physics of Biological and Complex Systems

of the Göttingen Graduate School of Neurosciences, Biophysics, and Molecular Biosciences (GGNB)

of the Georg-August University School of Sciences (GAUSS)

submitted by

Laura Turco

from Milano, Italy

Göttingen, 2017 


\section{Thesis Committee:}

\section{Dr. Marco Tarantola (Referee)}

Department of Fluid Dynamics, Pattern Formation and Biocomplexity Max Planck Institute for Dynamics and Self-Organization

\section{Prof. Dr. Claudia Steinem (Co-Referee)}

Institute for Organic and Biomolecular Chemistry

Georg-August-University Göttingen

\section{Prof. Dr. Stefan Luther}

Biomedical Physics Group

Max Planck Institute for Dynamics and Self-Organization

Other Members of the Examination BoARd:

\section{Dr. Karen Alim}

Biological Physics and Morphogenesis Group

Max Planck Institute for Dynamics and Self-Organization

\section{Prof. Dr. Sarah Köster}

Institute for X-Ray Physics

Georg-August-University Göttingen

Prof. Dr. Burkhard Geil

Institute for Physical Chemistry

Georg-August-University Göttingen

Date of oral examination: 28.06.2017 


\section{Affidavit}

Hereby, I declare that the presented thesis has been written independently and with no other sources and aids than quoted.

Parts of this thesis and few figures have been published in the journal article:

Rother, J.; Richter, C.; Turco, L.; Knoch, F.; Mey, I.; Luther, S.; Janshoff, A.; Bodenschatz, E.; Tarantola, M. "Crosstalk of cardiomyocytes and fibroblasts in cocultures" Open Biology 5150038 (2015)

Göttingen, 17.05.2017 



\section{Contents}

$\begin{array}{ll}\text { Abstract } & 1\end{array}$

1 Introduction $\quad 3$

1.1 Structure and Function of Cardiac Architecture . . . . . . . . . . . . . . 4

1.1.1 Laminar sheets . . . . . . . . . . . . . . . . . . . . 4

1.1.2 Collagen Network . . . . . . . . . . . . . . . . 5

1.1.3 Interstitial Fluid . . . . . . . . . . . . . . . . . 6

1.1.4 Cardiomyocytes . . . . . . . . . . . . . . . 6

1.1.4.1 Sarcomeric Cytoskeleton . . . . . . . . . . . 8

1.1.4.2 Non-Sarcomeric Cytoskeleton . . . . . . . . . . . . . 9

1.1.5 Cell-cell communication . . . . . . . . . . . . . . . . 10

1.1.6 Cell-ECM communication . . . . . . . . . . . . . . . 11

1.2 Cardiomyocytes Excitation-Contraction Coupling . . . . . . . . . . . . . 12

1.3 Mechanoelectrical Feedback . . . . . . . . . . . . . . . . . . . . 12

1.4 Left Ventricular Remodeling Post Myocardial Infarction . . . . . . . . . . 13

2 Materials and Methods $\quad 17$

2.1 Cells Culture and Sample Preparation . . . . . . . . . . . . . . . . . 17

2.1.1 Cell Culture Preparation . . . . . . . . . . . . . . . . . 17

2.1.2 Substrates Coating and Cell Seeding . . . . . . . . . . . . 18

2.1.3 Cell Staining . . . . . . . . . . . . . . . . . . . . 19

2.1.3.1 Fixation and Permeabilisation . . . . . . . . . . 20

2.1.3.2 Immunostaining . . . . . . . . . . . . . . 21

2.1.3.3 Fluorescent Phallotoxin Staining . . . . . . . . . . 21

2.2 Fluid Flow Stimulation . . . . . . . . . . . . . . . . . . . 22

2.2.1 Physiological Shear Stress in vitro Estimation . . . . . . . . . . . 22

2.2 .2 Setup . . . . . . . . . . . . . . . . 23

2.2.3 Experimental Procedure . . . . . . . . . . . . . . . . 25

2.3 Electric cell-substrate impedance sensing $\left(\mathrm{ECIS}^{\mathrm{TM}}\right) \ldots \ldots$. . . . . . 26

2.3.1 Cellular Impedance Modelling . . . . . . . . . . . . . . . . . 26 
2.3.2 Setup and Experimental procedure . . . . . . . . . . . . . . 29

2.3.3 Impedance Analysis . . . . . . . . . . . . . . . . . . . . . . 29

2.4 Microscopy . . . . . . . . . . . . . . . . . . . . 31

2.4.1 Confocal Laser Scanning Microscopy . . . . . . . . . . . . . . . 32

2.4.1.1 Experimental Procedure . . . . . . . . . . . . 32

2.4.1.2 Quantification of cellular connectivity . . . . . . . 33

2.4.1.3 Analysis of fibers orientation . . . . . . . . . . 34

2.4.2 Atomic force microscopy-based microrheology . . . . . . . . . . . 37

2.4.2.1 Setup and Experimental Procedure . . . . . . . . . . 40

2.4.2.2 Extraction of microrheological parameters . . . . . . . 40

2.4.3 Reflection Interference Contrast Microscopy . . . . . . . . . . . . 42

2.4.3.1 Setup and Experimental Procedure . . . . . . . . . . 42

2.4.3.2 Analysis of intensity and contact-area . . . . . . . 43

3 Results $\quad 45$

3.1 Mechanoelectrical Coupling and Mechanics in a Cardiac Fibrosis Model . 45

3.1.1 Coupling via Cellular Junction Proteins . . . . . . . . . . . . . 45

3.1.1.1 Electrical Junctions . . . . . . . . . . . . 46

3.1.1.2 Mechanical Junctions . . . . . . . . . . . . . 46

3.1.1.3 Impedance Measurements . . . . . . . . . . . . . . 50

3.1.2 Electromechanical Coupling . . . . . . . . . . . . 51

3.1.3 Microrheological properties of Cardiac Fibrosis Model . . . . . . . 53

3.2 Effect of shear stress on cardiac laminar sheets . . . . . . . . . . . . 55

3.2.1 Immediate-short term effect of shear stress on cellular morphology 56

3.2.2 Immediate-short term effect of shear stress on cellular contractility 59

3.2.3 Long-term effect of shear stress on cells morphology . . . . . . . . 60

3.2.4 Long term effect of shear stress on cellular contractility . . . . . . 68

4 Discussion $\quad 73$

4.1 Ventricular Remodeling: Fibrosis . . . . . . . . . . . . . . 73

4.2 Ventricular Remodeling: Effect of High Shear Stress . . . . . . . . . . . . 80

$\begin{array}{lll}5 & \text { Conclusions and Outlook } & 91\end{array}$

$\begin{array}{ll}\text { List of Figures } & 95\end{array}$

$\begin{array}{ll}\text { Bibliography } & 97\end{array}$

$\begin{array}{ll}\text { Glossary } & 108\end{array}$

$\begin{array}{ll}\text { Curriculum Vitae } & 111\end{array}$ 


\section{Abstract}

Left ventricular remodeling (LVR) after myocardial infarction usually results in sudden death attributable to the outbreak of arrhythmias. LVR is characterised by progressive cardiomyocytes slippage, interstitial space growth, increase of mechanical stress, hypertrophy, fibrosis and alteration of contractile function. The effect of changes in biophysical environment that occur in LVR on the mechanoelectrical coupling of cardiomyocites is poorly explored.

In this work, I studied the influence of changes such as the abundance of myofibroblasts in fibrotic conditions and the increased shear stress on cellular mechanical properties, morphology, contractility and connectivity. By using co-cultures of cardiomyocytes and myofibroblasts as an in vitro model system for fibrosis I observed that their communication occurs via both electrical and mechanical junctions. Co-cultures with low ratios of cardiomyocytes overexpressed electrical and mechanical junction proteins, Connexin43 (Cx43) and N-Cadherin (N-Cad), respectively, inducing alterations in the electrophysiology of cardiomyocytes: decrease of beating frequency and outbreak of spiral waves. The enhanced expression of N-Cad shows that myofibroblasts may influence the function of cardiomyocytes by applying contractile forces via mechanical junctions. $\mathrm{N}$-Cad in turn is responsible for the transmission of contractile forces between myofibroblasts throughout the fibrotic scar. AFM-based microrheological measurements revealed that viscoelastic properties change under fibrotic conditions. Co-culture was observed to be stiffer than both cardiomyocytes and fibroblasts monocultures and was characterised by a solid-like behaviour at almost all frequencies.

Another physiological change that occurs in LVR is the increase of mechanical load on cardiomyocytes, in particular shear stress, which is often neglected in in vitro and in silico models. By combining impedance spectroscopy and optical microscopy I have shown that high values of shear stress stimulation lead to an immediate decrease of cell-substrate distances at the flow onset, cell spreading up to 48 hours, and a gradual reorientation of the actin fibers along the direction of the flow that took upto 108 hours. Additionally, beating frequency and cell-cell connectivity of cardiomyocytes increased under shear stress. Furthermore, contraction of cardiomyocytes synchronised in the presence of shear stress. Flow stimulation increased the beating frequency of the cardi- 
omyocytes and myofibroblasts co-cultures similar to the monocultures, and for the first time, I observed contractility in co-cultures with 9:1 ratio of myofibroblasts after shear stimulation, whereas the non-sheared co-cultures did not show any activity.

In conclusion this work proves that stimulating cardiomyocytes with high shear stress is a reliable in vitro pathological model to reproduce conditions similar to the in vivo situation. Furthermore, experimental and modelling studies used to understand pathophysiology during LVR should take into account the presence of high shear stress and its influence on mechanoelectrical coupling and cellular morphology. 


\section{Introduction}

The aim of this work is to study the effect of changes in the biophysical environment on cells during left ventricular remodeling after myocardial infarction (MI). Post-infarction remodeling is divided into two stages: early phase and fibrosis, as will be explained in detail at the end of this chapter. A deep understanding of the mechanisms involved in this phenomenon may be useful to develop new therapeutic strategies to prevent the usual outbreak of arrhythmia after MI. Current therapies targeting the post-infarction remodeling are based on mechanical or pharmacological interventions, but both have drawbacks. Mechanical approaches such as surgery are invasive and are performed on patients with certain eligibility criteria [1]. On the other hand, neurohormonal inhibitor drugs, which are supposed to reduce hypertrophy or fibrosis, are effective only on patients with a mild infarct and they can increase the risk of hypotension. A recent promising approach based on regenerative medicine aims to restore cardiac function with the help of stem cells reprogrammed into healthy cardiomyocytes. The efficacy of this novel therapy has not yet been demonstrated in preclinical models [2]. Studies that employ new in vitro cellular and tissue scale model systems will be extremely useful to investigate a complicated dynamic process such as ventricular remodeling. Such a system will allow to investigate individually the effect of mechanical, biochemical, or electrical changes on cardiac cells within controlled conditions.

During ventricular remodeling post MI, cardiac cells are exposed to increased mechanical loads, including high wall stresses and high shear stresses [3]. Several groups applied stretching to cardiac cell monolayers showing the relationship between mechanoelectrical feedback and cardiac diseases caused by increased mechanical loading [4-8]. It has been observed that cyclic stretching upregulates the expression of Connexin43 (Cx43) in neonatal rat ventricular myocytes (NRVMs) related to an increase of secretion of angiotensin II [6] and growth factors [7]. Another group found that NRVMs align and localise Cx43 at the longitudinal extremities of the cells [7]. Yang and coworkers reported live-cell observations of sarcomeric addition, the major molecular event that occurs in hypertrophic conditions, in NRVMs under uniaxial static stretch [8]. Although many efforts have been done to investigate the influence of stretching on cardiomyocyte physiology, the of effect shear stress on the morphology and contractility of the cells has 
not been extensively studied. Few studies in this direction have reported a decrease in action potential duration [9] and intracellular calcium transient [10] in atrial neonatal cardiomyocytes when stimulated by shear stress for few minutes. It has also been shown that NRVMs exhibit higher beating frequency [11] and an increase in gap and adherens junctions [12] when continuous and oscillatory shear stress was applied, respectively. These studies analysed only the short-term effect of high shear stress stimulation. Thus, a long-term response to elevated shear stress, which is more realistic if we refer to the early phase of ventricular remodelling conditions, remains unknown.

In this work I investigated the short- and long term effect of high shear stresses on cellular morphology, contractility, and connectivity. In order to complete the study of the environment changes in ventricular remodeling on cardiac cells, I also analysed the mechanical properties of fibrotic tissues and the communication between cardiomyocytes and myofibroblasts, correlating it with electromechanical coupling.

Before going into the details of the work, I present a brief overview of the fundamentals that will aid one to understand the results presented in this thesis and the discussion that follows.

\subsection{Structure and Function of Cardiac Architecture}

\subsubsection{Laminar sheets}

The ventricular myocardium has a complex architecture in which cardiac cells are organised in layers or laminar sheets. These sheets are mainly formed by fundamental contractile cells, called cardiomyocytes. However, in the myocardium other cell types can be found, such as fibroblasts, which represent the majority of the cells, endothelial cells, and smooth muscle cells. The sheets are on average three to five cells thick and continuously branch in each direction throughout the ventricular wall. Cardiomyocytes are oriented in different directions (fiber direction) within the myocardium: at the epicardial and subendocardial surface the orientation is $-60^{\circ}$ and $+90^{\circ}$, respectively with respect to circumferential direction [13]. The ventricular sheet architecture is represented in figure 1.1A-D. Within laminar sheets, cardiomyocytes are tightly coupled both mechanically and electrically (figure 1.1B), while neighbouring layers are separated by cleavages but interconnected on a larger scale (figure 1.1C). The laminar architecture of ventricular myocardium has mostly a mechanical function. During heart relaxation and contraction the sheets slide against each others and along the cleavage planes resulting in a rearrangement of the ventricle structure providing a basis for wall thickening changes at the end of systole. Wall thickening is an important component of the normal cardiac cycles because it contributes to the stroke volume [14]. Pope and coworkers demonstrated that the orientation of ventricular laminar sheets coincides with planes 
of maximum shear deformation, suggesting that relative sliding between laminae allows deformations throughout the cardiac cycle [15]. The laminar structure also affects the electrical properties and enable a fast propagation of electrical waves in the direction of the fibers through branches, an intermediate and slowest propagation along the sheet axis and sheet normal axis respectively (figure 1.1A) [16].

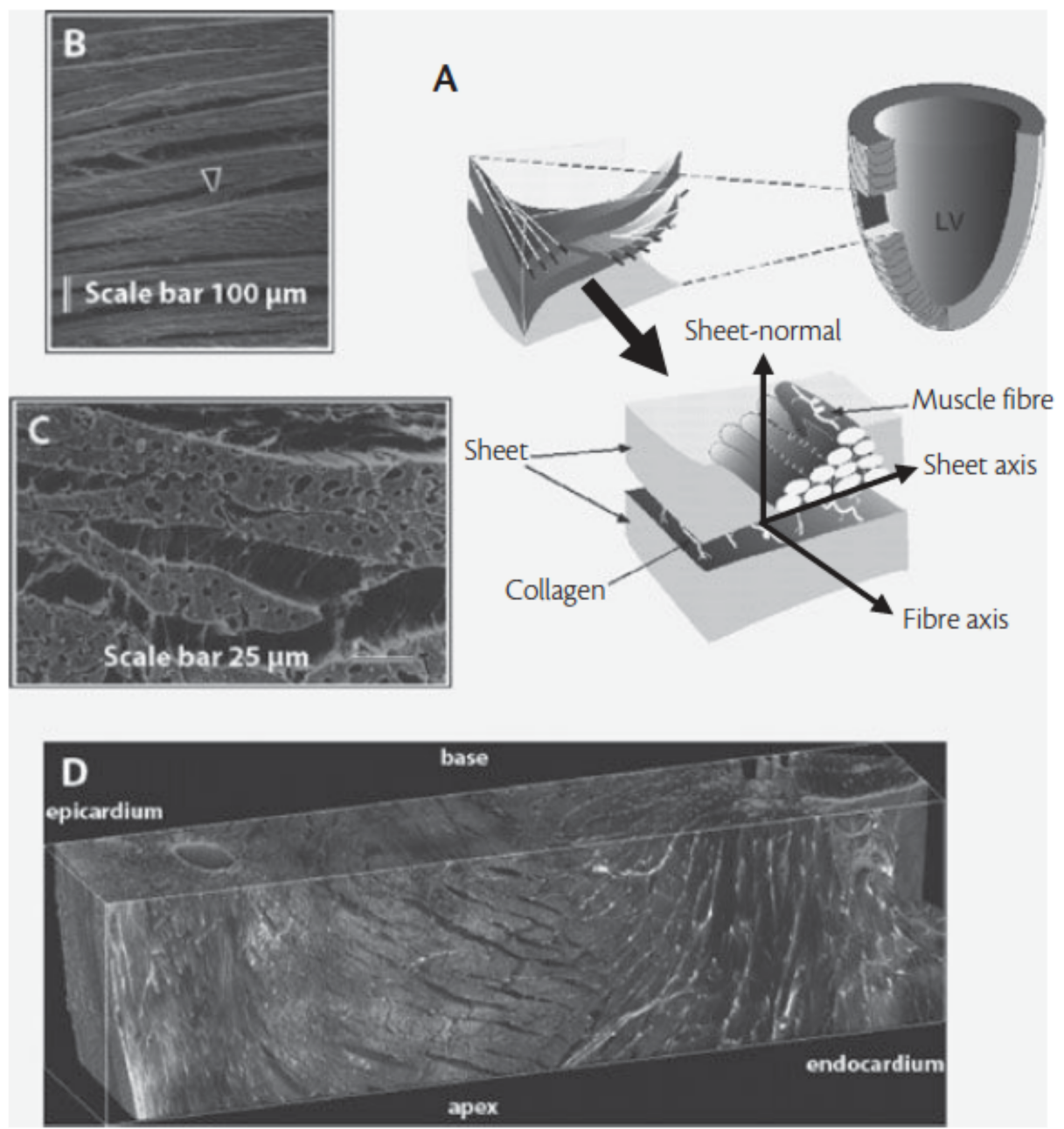

Figure 1.1: The laminar sheet architecture of ventricular myocardium. A: Schematic of ventricular microstructure: sheets with different fiber orientations indicated with white rods (left); cardiomyocytes tightly coupled within a sheet and the structural axes: fiber, sheet, and sheet-normal axis. B: Scanning electron micrograpth of midwall ventricular segment in a dog heart in the sheet-normal and sheet axis plane. Branching between adjacent laminar sheets is indicated by arrowhead. C: Scanning electron micrograph of midwall ventricular segment in a dog heart in the sheet-normal and fiber axis plane. D: 3D reconstruction using confocal microscopy of myocytes arrangement in a transmural segment of normal rat heart. Dimensions are $4.3 \times 1.1 \times 0.9 \mathrm{~mm}$. Picture taken from [13]

\subsubsection{Collagen Network}

The organisation and the function of myocardium is highly related to the cardiac extracellular environment, the extracellular matrix (ECM). Cardiac ECM consists of collagen 
I, II, and IV, laminin, fibronectin, and proteoglycans [17]. The tight coupling of cardiomyocytes within the sheet is provided by a network of connective tissue, extracellular collagen fibers, which is also responsible for a weaker coupling between adjacent sheets. Collagen, the main structural component of cardiac ECM, is classified as epimysium, surrounding the all muscle; endomysium, the tight network that interconnects single cardiomyocytes and capillaries; and perimysium, surrounding and connecting laminar sheets [17]. The latter contributes to yielding the laminated structure to the ventricle. Wang and coworkers used extended- volume confocal microscopy to visualised 3D perimysial collagen in a rat heart [13]. They observed three forms of perimysial collagen: two meshes of collagen fibers, one surrounding the sheets and one spanning in the cleavage plane between sheets, and a long cords of collagen aligned with the myocardial fiber axis (figure 1.1A). The function of the collagen meshes is to give structure to the laminar architecture and to enable the rearrangement or shearing of cardiac sheets during contraction. The longitudinal cords of collagen are responsible for the generation of passive tension against extension along the myofibers axis. Thus, cardiac connective tissue is essential for maintaining the structure of cardiac laminae and limiting the extension during diastole [13].

\subsubsection{Interstitial Fluid}

The cardiac muscle contains connective tissue and cells, surrounded by fluid-filled extracellular space. This fluid is called interstitial fluid and plays an important role in the regulation of both biochemical and biophysical signals in the extracellular space. Such as the microcirculation which provides the necessary transport of biomolecules. Indeed it regulates the mass exchange between cells and the surroundings, including delivering of nutrients and removal of metabolic waste. Interstitial fluid is also involved in intercellular communication, distributing biomolecules, e.g. cytokines, growth factors, and cations, in the extracellular space. It creates a specific mechanical environment by applying shear stress and hydrostatic pressure to cells which are crucial for cellular functions [18].

\subsubsection{Cardiomyocytes}

The fundamental contractile cells of the myocardium are the cardiomyocytes. They are cylindrical cells with diameter and length of about $10-15 \mu \mathrm{m}$ and $100 \mu \mathrm{m}$, respectively [19]. They are connected to each other along the longitudinal axis and arranged into a network [17]. This complex architecture is defined by the sub-cellular organisation of $(i)$ the intercalated disc, $(i i)$ the sarcolemma, and (iii) the cytoskeleton (figure $1.2)$. 


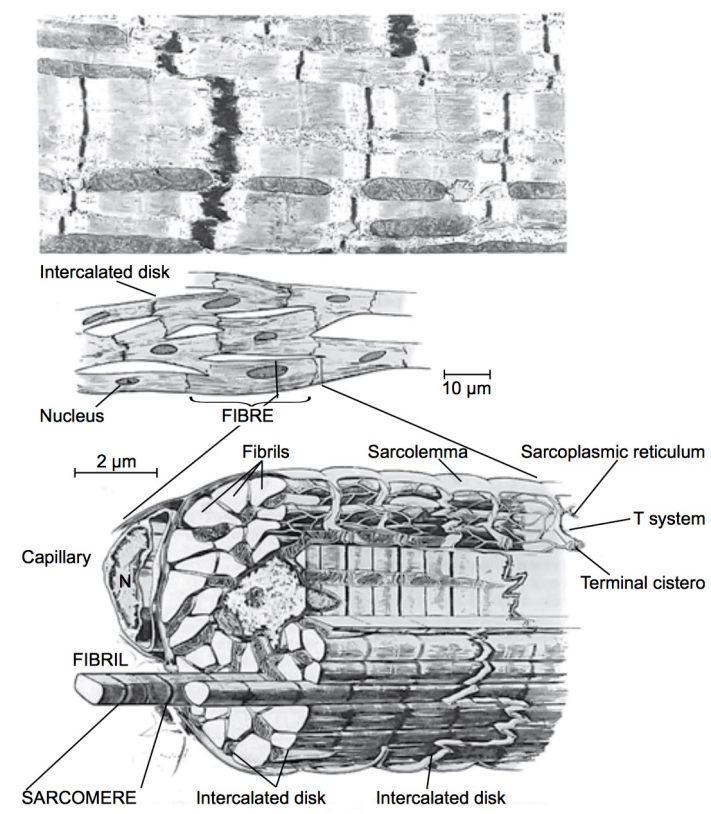

Figure 1.2: Cardiac muscle morphology. Top: Electron microscopy of a cardiac muscle. Middle: Illustration of cardiac muscle syncytium. Bottom: Illustration of a part of cardiac muscle cell. Illustration taken from $[17]$

- The intercalated disks represent the area where two cardiomyocytes are connected to each other along the longitudinal axis. They are structural parts that enable the transmission of contractile force and allow the cells to work as a single functional organ. They also serve as a low resistance component that allows rapid conduction of action potential through cardiac tissue. Three types of junctional complexes provide proper connection and communication between cardiomyocytes: desmosomes, adherens junctions, and gap junctions[17].

- The sarcolemma is the plasma membrane surrounding the cardiomyocytes whose function is to provide a barrier for diffusion. It is composed of a lipid bilayer and contains proteins, receptors, pumps, and ion channels that are fundamental for the propagation of the action potential and the cell contraction. An important receptor that allows the neurohormonal system to regulate cardiac function is $\beta 1$-adrenoreceptor, at which norepinephrine binds and stimulate beating rate and force of contraction [20]. The sarcolemma forms invaginations into the cardiomyocyte forming the trasversal tubules (T-tubules). These extensions allow the L-type calcium channels to be close to the sarcoplasmatic reticulum, as shown in figure 1.2, making T-tubules an important structural element involved in the excitation-contraction coupling [21].

- The cytoskeleton is the structure that provided mechanical support to the cell and it is involved in mechanotransduction. It is responsible for the propagation 
of mechanical signals throughout cardiomyocytes triggering several functional responses. The components of the cytoskeleton can be divided in sarcomeric and non-sarcomeric elements. The former include thin and thick filaments involved in the generation of contractile forces whereas, the latter are the filaments that take part in transmission of forces and signals form both intracellular and extracellular space [17].

\subsubsection{Sarcomeric Cytoskeleton}

A sarcomere represents the basic contractile unit of cardiomyocytes, approximately $2.2 \mu \mathrm{m}$ long, and consists of thin and thick filaments confined between two $\mathrm{Z}$ disks (figure 1.3). The thin filaments are composed by the proteins complex of actin, $\alpha$ tropomiosin, C-,I-,T-troponins. Actin is a globular proteins that in the sarcomere is arranged in two tangled chains at which the thick filaments attach. Tropomyosin is connected to actin strands, stabilising them and preventing their depolymerisation. Troponin binds to tropomyosin with its sub-unit $\mathrm{T}$, and has an affinity for both $\mathrm{Ca}^{2+}$ and actin. The sub-unit $\mathrm{C}$ binds to $\mathrm{Ca}^{2+}$ during the excitation-contraction coupling and the sub-unit I to the actin, suppressing the actin-thick filament interaction. The thick filaments consist of several hundreds of myosin molecules, associated in a parallel array. Myosin consist of two heavy globular chains and two pairs of light chains, which coil around each other to forms dimers. The globular heads bind to the actin, forming cross-bridges between the thick and the thin filaments. Myosin heads bind also to an enzyme that hydrolyses ATP, required for actin-myosin cross- bridge formation and that provides the energy for the filaments sliding, as described later.

Several sarcomeres connected in series form myofibrils that run along the longitudinal axis of the cardiomyocytes. The $\mathrm{Z}$ disks connect myofibrils and transfer the contractile force into costameres, sub-sarcolemmatic structures that communicate with the ECM. In the $\mathrm{Z}$ disks proteins such as $\alpha$-actinin hold together and stabilise the thin filaments of parallel sarcomeres.

The sarcomere is divided in three zones and this partition is displayed in figure 1.3: A-band, is the area where actin and myosin filaments overlap and contains the entire length of a single myosin; I-band, is the zone where actin is not superimposed by myosin; H-band, consist of the part where myosin is not superimposed by actin. Within this band the M-line is where the myosin filaments are connected.

Actin and myosin filaments maintain their respective positions through to a protein named titin, also known as connectin. It binds the $\alpha$-actinin and crosses half sarcomere until the M-line. It also gives flexibility to the myofibril, acting as a spring and takes part in the recovery of sarcomere initial length after contraction [17]. 


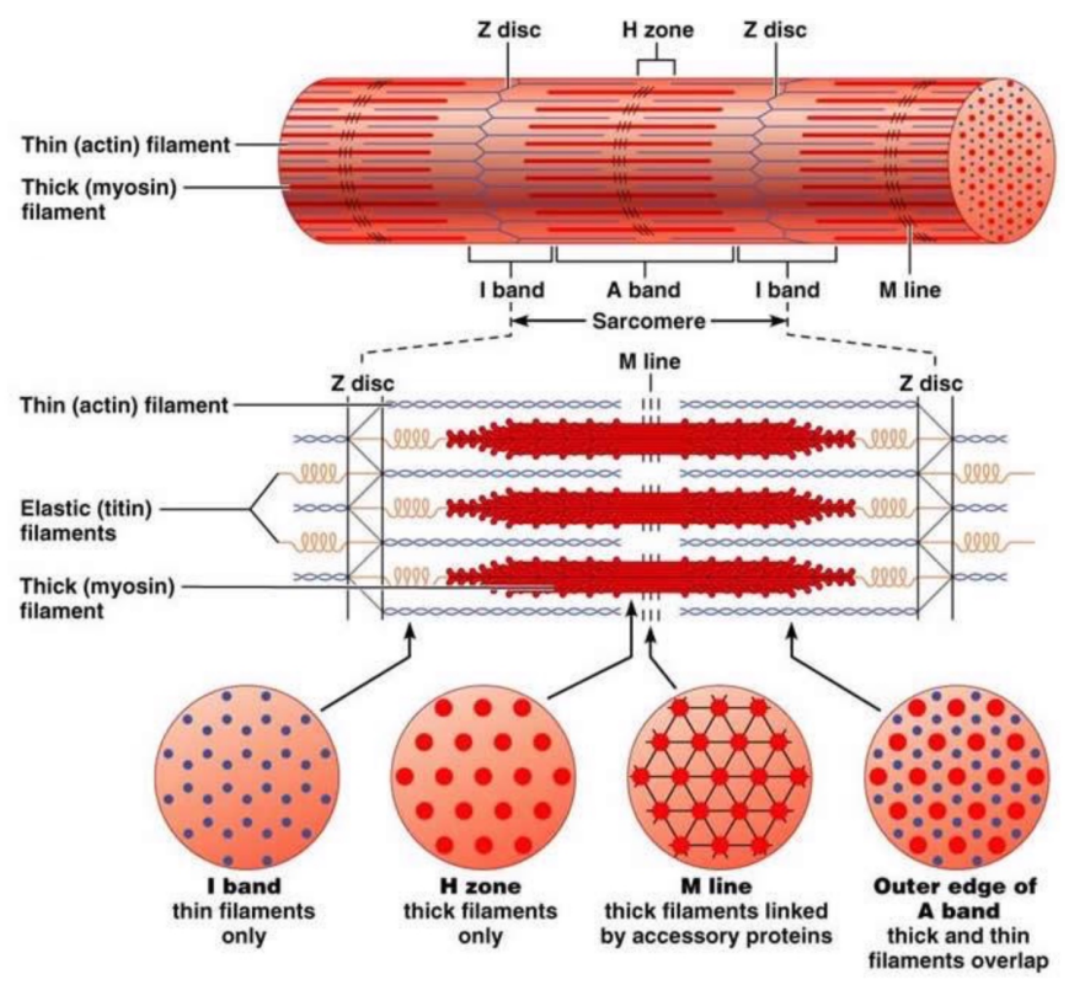

Figure 1.3: Sarcomere structure: the arrangement of thin and thick filaments is illustrated [22]

\subsubsection{Non-Sarcomeric Cytoskeleton}

The filaments that are not involved in the sarcomeric contraction but support subcellular structure, reorganise the cytoskeleton, regulate the topology of the cell membrane, and propagate biochemical or mechanical signals are microtubules, intermediate filament and non-sarcomeric actin fibers.

- Microtubules are very dynamic structures with multiple functions. They are polymers constituted by $\alpha$ - and $\beta$-tubulin heterodimers and are localised mostly around the nucleus along the longitudinal axis of cardiomyocytes. In healthy cardiomyocytes the percentage of tubulin that is polymerised is only $30 \%$ whereas the rest is in the cytoplasm in a non-polymerised form. Microtubules can modify the cytoskeletal network by de-polymerising and re-polymerising rapidly. They are responsible for the intracellular transport, acting as a substrate for motor proteins that carry vesicles through the cells. They also have a structural function connecting myofibrils to mitochondria, therefore contributing to cell stability. Microtubules are involved in biological processes that require a reorganisation of the cytoskeleton such as mitosis in which they drag chromosomes to the edges of the new forming cells. They may also remodel in response to extracellular stimuli. It was reported that pressure overload hypertrophy is associated with microtubules hyper-polymerisation, subsequantial increase of the polymerised tubulin in the cell 
and the viscosity of the cytoplasm [17]. A recent study in vitro showed that microtubules can provide mechanical resistance to the cell through the interaction with sarcomere and that they buckle during contraction and unbuckle during relaxation. This implies that microtubules are mechanically coupled to the sarcomeres and that they can propagate forces throughout the cytoskeletal network [23]. In summary microtubules, beside their structural and delivery function play a crucial role in sensing both intracellular and extracellular forces.

- Actin filaments are also present outside the sarcomeres and they are softer than the above mentioned microtubules. They form a network connecting the $\mathrm{Z}$ disk through $\alpha$-actinin with several parts of the costameres and intercalated disks. The function of non-sarcomeric actin in cardiomyocytes is not clear, it may provide a passive structural role to maintain the cell shape and anchor to other cytoskeleton proteins $[17,24]$.

- Desmins are one type of intermediate filaments and they are necessary for the structural integrity of cardiomyocytes to maintain cellular organelles in a specific position. They are transversely distributed along the myofibril and connect the nuclear membrane to the $\mathrm{Z}$ disks and the sarcolemma. The arrangement of desmins is modified by external mechanical tension and affect nuclear functions such as gene transcription [25].

The structure of non-sarcomeric cytoskeleton is sensitive to mechanical exogenous and endogenous inputs. It is sensitive to environmental changes and is able to dynamically remodel to sustain the mechanical load. Thus, the cytoskeleton plays a central role in the conversion of mechanical forces into biochemical or electrical signals (mechanotransduction).

\subsubsection{Cell-cell communication}

The transmission of contractile forces and action potential from one cardiomyocyte to another occurs at the intercalated disks through gap junctions, adherens junction and desmosomes. Gap junctions mediate the chemical and electrical coupling of neighbouring cells, whereas desmosomes and adheres junctions form the mechanical connections. The latter are linked to actin cytoskeleton and to intermediate filaments [17].

- Desmosomes are molecular complex anchored to the desmins of connected cells. They prevent the detachment of cells under contraction activity.

- Adherens junctions link to the actin fibers of the non-sarcomeric cytoskeleton and provide a strong mechanical connection between cardiomyocytes. They serve also as attachment points to myofibrils enabling the transmission of contractile 
force between neighbouring cells. The adherens junctions in cardiomyocytes are mainly composed of $\mathrm{N}$-Cadherin which is a transmembrane protein that connects cardiomyocyes, separating their membrane by $20 \mathrm{~nm}$, at distances $0.2-0.5 \mu \mathrm{m}$ apart [26]. Studies have demonstrated a possible role of cadherins as mechanosensors since they actively strengthened cell-cell junctions in response to traction forces [27]. Thus adhesion junctions may be responsible for the bi-directional transmission of cytoskeletal tension between cells via N-Cadherin. In neonatal cardiomyocytes $\mathrm{N}$-Cadherin is also localised in costameres, indicating the formation of lateral cell-cell mechanical junctions that can sense mechanical stresses along the transverse axis [28].

- Gap junctions directly connect two neighbouring cell forming a channel through which metabolites, waters and ions (e.g. $\left[\mathrm{Ca}^{2+}\right]$ ) up to a molecular mass of 1000 Da can diffuse [29]. This allows electrical and metabolic coupling of cardiomyocytes. They are formed by two adjacent hemi-channels (connexons) made of connexins that connect the intracellular space of the neighboring cells. In ventricular myocardium connexin 43 is predominant while connexin 45 is present only in the endocardium [30]. In cardiomyocytes gap junctions are key structures for a proper propagation of the electrical impulse which triggers the coordinated contraction of connected cells. In immature cardiomyocytes gap junctions are distributed along the whole periphery and during maturation localise with the intercalated disks to ensure rapid conduction of action potential [31]. Disruption of this organisation is associated with abnormal conduction and arrhythmias [32].

\subsubsection{Cell-ECM communication}

Cardiomyocytes communicate not only with other cells but also with the surrounding network at which they are anchored. This communication is mediated by transmembrane integrin protein receptors which form focal adhesion complexes. Integrins are dimers consisting of $\alpha$ and $\beta$-subunits and different combinations of these subunits define the specificity of the receptors to the ECM components [33]. Integrins interact inside the cell with the sarcomeres via proteins such as talin, vinculin, and $\alpha$-actinin. Extracellularly they attach to the ECM components such as collagen and fibronectin. Changes in both composition of ECM and integrins expression are transmitted via cytoskeleton to the cell. Indeed, the integrins, besides providing structural support binding the cells to the ECM, are involved in many cellular processes such as proliferation and signaling depending on which type of integrins are expressed by myocytes [27]. 


\subsection{Cardiomyocytes Excitation-Contraction Coupling}

The electrical activation of cardiomyocytes leads to a mechanical contraction through activation of a $\left[\mathrm{Ca}^{2+}\right]$-dependent process . This process, known as excitation-contraction coupling, is schematised in figure 1.4. The excitation-contraction coupling starts with the membrane depolarisation at action potential activation. This leads to the opening of voltage-gated L-type calcium channels, localised in the T-tubules, and to the influx of $\left[\mathrm{Ca}^{2+}\right] .\left[\mathrm{Ca}^{2+}\right]$ binds to a receptor $(\mathrm{RyR})$ on the surface of the sarcoplasmic reticulum (SR), an intracellular membrane network that regulates the concentration of $\left[\mathrm{Ca}^{2+}\right]$ in the intracellular space. SR releases $\left[\mathrm{Ca}^{2+}\right]$ increasing the amount of free ions in the cell. $\left[\mathrm{Ca}^{2+}\right]$ binds to the troponin-C (Tn) filament. This leads to a conformational change in troponin- $\mathrm{C}$ that induces tropomyosin ( $\mathrm{Tm}$ ) to shift and expose the activated myosin to actin allowing them to form a cross-bridge. After binding, myosin slides the actin filament towards the centre of the sarcomere (power stroke). During relaxation the $\left[\mathrm{Ca}^{2+}\right]$ is then pumped back into the SR via an ATP-dependent pump (SERCA) and expelled via sodium-calcium exchanger (NCX) [34].

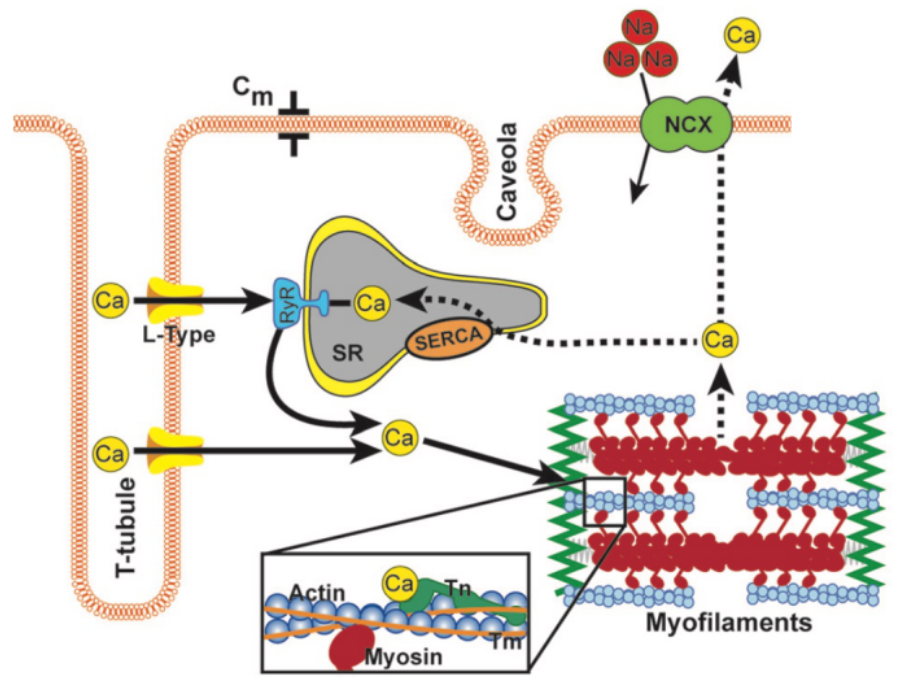

Figure 1.4: Cardiomyocyte Excitation-Contraction Couplin. Figure taken by [34].

\subsection{Mechanoelectrical Feedback}

Besides the excitation-contraction coupling, mechanical loads also influence cardiac electrical activity [35]. At cellular level a mechanical alteration in the extracellular environment (figure1.5) induces changes in the length and tension of cardiomycytes which feedbacks the excitation that leads to control the contraction [36]. Cardiomyocytes are continuously exposed to mechanical stresses and indeed several studies have characterised the response of cells to cyclic stretch. It has been shown that mechanical stretch 
alter action potential duration, conduction velocity and cellular orientation [5, 37]. As already mentioned above, mechanoelectrical feedback has been characterised by applying stretching to the cells. However, during cardiac cycle cardiomyocytes are exposed to other mechanical forces such as intramyocardial pressure and shear stress. The latter arises from the relative slide of cardiac laminar sheets during cardiac cycle and from interstitial fluid movement. The contribution of both pressure and shear stress remains largely unstudied. Cardiac mechanoelectrical feedback is thought to have an important role in pathological conditions. Alteration in cardiac mechanics at both organ and cellular level has been observed to promote gap junction remodeling increasing susceptibility to arrhythmia [4].

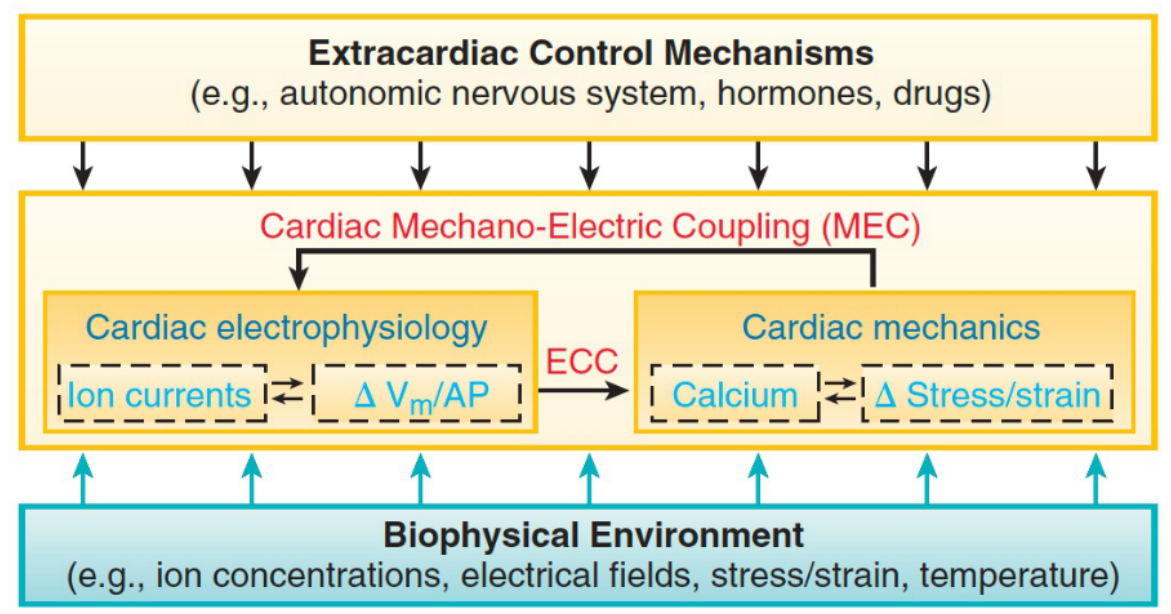

Figure 1.5: Schematic view of electromechanical integration. Electrical activity (cardiac electrophysiology dependent on ion currents, membrane potential $\Delta \mathrm{V}_{m}$, and action potential AP) induces contraction (cardiac mechanics dependent on calcium) via excitation-contraction and mechanical loads affect back cardiac electrophysiology via mechanoelectrical coupling. Electromechanical interactions are modulated by biophysical environment and extra cardiac control systems. Figure taken by [38].

\subsection{Left Ventricular Remodeling Post Myocardial Infarction}

The acute loss of myocardium after an infarction is followed by dilatation of left ventricle and changes in cardiac function. The death of cardiomyocytes and the modified mechanical conditions trigger mechanisms mediated by intra- and extra-cellular signaling such as inflammatory response, cell hypertrophy, and the formation of collagen scar. This dynamic process is known as left ventricular remodeling and is divided into two phases [39].

In early phase, within 72 hours after MI, the infarct zone expands and become thinner because of the degradation of the collagen network that holds cardiomyocytes together forming sheets. Thus, cells lose anchoring points with ECM that results in cardiomyocytes slippage and loss of their normal parallel alignment [40]. Slippage of cardiomyo- 
cytes causes thinning and dilatation of the myocardial wall and an increase in systolic wall stress that serve as a stimuli for hypertrophy in the non-infarcted cells [39]. Slippage leads also to the growth of interstitial space and consequently higher shear stresses on the cells during cardiac contraction. Initially this is an adaptive response, but if persists it results in an increase of neurohormonal activation [3], such as angiotensin II and norepinephrine. This stimulates expression of contraction proteins which assembly in new sarcomeres resulting in the elongation of cardiomyocytes [3, 41]. This stage of remodeling involves alterations in ventricular architecture that is followed by an attempt to distribute the increased wall stresses.

In the late phase of remodeling, named fibrosis, the released chemical factors such as angiotensin II and tumor growth factor- $\beta 1$ (TGF- $\beta 1$ ), influence the function of the most numerous cell type in the myocardium: the fibroblasts. Once activated by these chemical factors, fibroblasts proliferate and migrate toward the damaged area. TGF- $\beta 1$ is one of several promoter of fibroblast differentiation into myofibroblasts. Myofibroblasts start to synthetise an excess of collagen and other ECM proteins forming a scar. Unlike

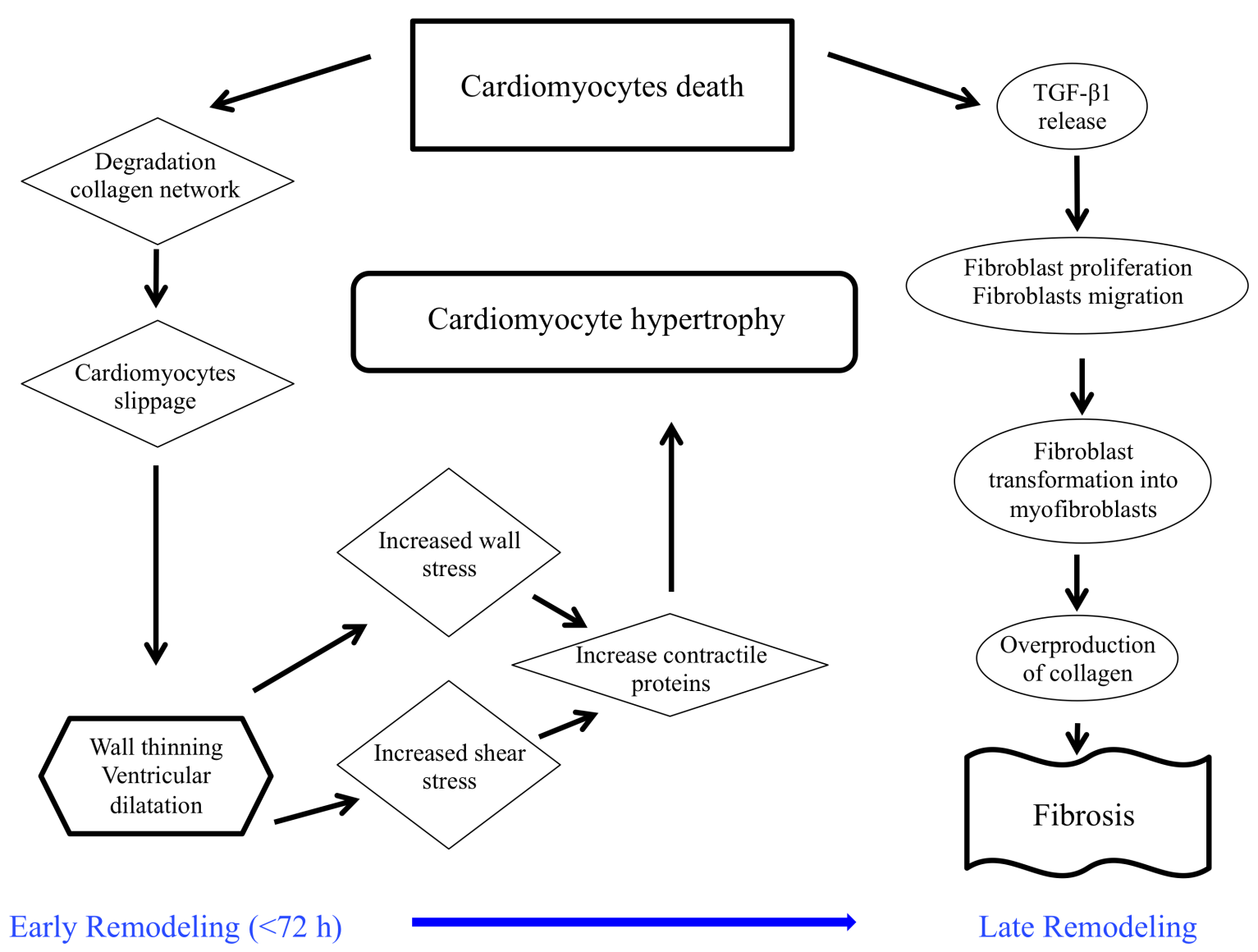

Figure 1.6: Diagrammatic representation of pathophysiology of ventricular remodeling inspired by work of Sutton and coworkers [39] 
fibroblasts, myofibroblasts express a contractile protein: $\alpha$-smooth muscle actin $(\alpha-$ SMA) [42]. This enable them to provide mechanical tension to remodel the ECM and close the wound to reduce the scar size. The scar at the infarct site is form to stabilise the wall stress and prevent further deformation. In figure 1.6 some of the factors involved in both early and late stage of ventricular remodeling are illustrated. 


\section{Materials and Methods}

This chapter presents the materials and methods used to study the connectivity between cardiomyocytes and/or fibroblasts and their viscoelastic properties in a cardiac fibrosis model. Additionally, techniques employed to investigate the effect of shear stress on laminar cardiac sheets are explained.

\subsection{Cells Culture and Sample Preparation}

\subsubsection{Cell Culture Preparation}

In this work I used monocultures of neonatal ventricular cardiomyocytes, cardiac fibroblasts and co-cultures with different percentage of these two cell types as a model system. The cells were isolated from hearts of 1-2-day-old neonatal Wistar rats, provided from animal care facilities of the Max Planck Institute for Experimental Medicine, Göttingen, Germany. In order to isolate cardiomyocytes, the ventricles of the rat were minced and enzymatically digested several times for $15 \mathrm{~min}$ at $37^{\circ} \mathrm{C}$ with collagenase II $(300 \mathrm{U}$ ml-1, Worthington, USA). For fibroblasts isolation, the cardiac tissue was digested with $0.05 \%(\mathrm{w} / \mathrm{v})$ trypsin in $1 \mathrm{mM}$ EDTA (ethylenediaminetetraaetic acid) in PBS (phosphate buffered saline) for $20 \mathrm{~min}$ at $37^{\circ} \mathrm{C}$ together with glass pearls under constant stirring. Afterwards, the fibroblasts were collected by centrifugation, diluted in cryopreserving medium consisting of 20\% (v/v) DMSO (dymethylsulfoxide), $40 \%$ FCS (fetal calf serum) and 40\% Medium 1 (50\% FCS, 50\% fibroblasts culture medium) and stored in liquid nitrogen. The frozen cells were thawed, plated on culture flasks and detached from the substrate when the cell layer $70 \%$ of confluence using $1 \mathrm{mM}$ EDTA in PBS for 5 min at $37^{\circ} \mathrm{C}$. After washing with PBS, the fibroblasts were collected by centrifugation.

Fibroblasts and freshly isolated cardiomyocytes were counted with a Neubauerimproved counting chamber (Marienfeld, Germany) and then re-suspended in a culture medium that we referred to as "Day 0-2" and re-suspended according to the required cardiomyocytes/fibroblasts ratio for each experiment. The composition of culture media used in this study are summarised in table 2.1 


\section{Cell media composition}

\begin{tabular}{ll}
\hline Culture medium "Day 0-2" & Dulbeccos's Modified Eagle's Medium (DMEM)/F12 \\
& $+10 \%$ Fetal Bovine Serum \\
& $+1 \%$ penicillin \\
Culture medium "Day 3" & DMEM/F12 \\
& $+6 \%$ Fetal Bovine Serum \\
& $+1 \%$ penicillin \\
& $+1 \%$ norepinephrine \\
& \\
Fibroblasts culture medium & \\
& $+10 \%$ Fetal Bovine Serum \\
& $+1 \%$ penicillin \\
& $+1 \%$ L-Glutamin \\
\hline
\end{tabular}

Table 2.1: Composition of cell media used for preparation and cultivation

\subsubsection{Substrates Coating and Cell Seeding}

Cells were seeded on different substrates according to the different experiment and protocol. In this work I used round glass slides, for rheological measurements, $\mu$ Slide $\mathrm{VI}^{0.4}$, Sticky-Slide $\mathrm{VI}^{0.4}$ and ECIS flow arrays $1 \mathrm{E}$ for flow based assays. In order to promote cell adhesion all the substrates were coated under sterile conditions with $0.02 \%$ gelatin and $0.5 \%$ fibronectin (v/v\%) [43] (Sigma-Aldrich, Germany) and incubated at $37^{\circ} \mathrm{C}$ for at least 1-2 hours, before cells seeding. The different substrates, their functionalisation and seeding procedures are listed below:

- Glass slides (3.5 cm, Asylum Research, Santa Barbara, CA, USA) were autoclaved, placed into a petri dish (60 mm diameter), and incubated with $208 \mu \mathrm{L}$ of coating solution. After washing the glass slides with fresh "Day 0-2" medium, cells were seeded with a density of $5 \times 10^{5}$ cells/slide and kept for 5 days at $37^{\circ} \mathrm{C}$ in $5 \%$ $\mathrm{CO}_{2^{-}}$humidified incubator until AFM-experiments (described in chapter 2.4.2). The culture medium was daily exchanged and from the third day after seeding was switched to a culture medium that we referred to as "Day 3". Since the adhesion times of cardiomyocytes and fibroblasts are different (3.5 h, $0.5 \mathrm{~h}$, respectively) [43], the samples for the assay with different ratios of cardiomyocytes and fibroblasts were prepared by adding the fibroblasts $24 \mathrm{~h}$ after the seeding of cardiomyocytes. This procedure avoids the formation of a cell double layer.

The substrates of the channels used for the flow assays were coated with the coating solution by using a $1 \mathrm{~mL}$ syringe. Two of such syringes were used as reservoir during incubation to avoid evaporation.

- $\mu$-Slide VI ${ }^{0.4}$ Luer (400 $\mu \mathrm{m} / 100 \mu \mathrm{L}$ height/volume, Ibidi, Martinsried, Germany) was used for both static and flow stimulated cell culture. $300 \mu \mathrm{L}$ of coating solu- 
tion was applied into the channels and incubated. The cell solution $\left(5 \times 10^{5}\right.$ cells/channel) was injected after 24 hours. For both static and flow stimulated culture, the slides were kept in incubation for 5 days as described above for the glass slides. At the end of the shear stress experiments cells were fixed and stained as described in subchapter 2.1.3. Fibroblasts were added to the culture, as previously explained.

- sticky-Slide I ${ }^{0.4}$ Luer $(450 \mu \mathrm{m} / 102.5 \mu \mathrm{L}$ height/volume, Ibidi, Martinsried, Germany) was used in combination with Glass Coverslips D 263 M Schott $(2.5 \times$ $7.5 \mathrm{~cm}, \mathrm{n}=1.523$, Ibidi, Martinsried, Germany). The coverslips were autoclaved before usage. The two parts were assembled together via a biocompatible doublefaced adhesive tape under sterile conditions, placed in a petri dish and incubated overnight at $37^{\circ} \mathrm{C}$ to improve the bonding. Once assembled, the slides were coated and seeded according to the same procedure used for the $\mu$-Slide VI ${ }^{0.4}$ Luer. The cells were then incubated in static conditions for 1 day before starting the shear stress stimulation. Due to the optical properties of the glass, these slides were used for Reflection Interference Contrast Microscopy (RICM) measurements.

- ECIS Flow Array 1E (400 $\mu \mathrm{m} / 100 \mu \mathrm{L}$ height/volume, Ibidi, Martinsried, Germany) was used for impedance measurements. The electrodes inside the channel were coated, seeded and incubated as explained for $\mu$-Slide VI ${ }^{0.4}$ Luer.

ECIS 8W1E array was used for impedance measurements of cardiomyocyte and fibroblast co-cultures by Jan Rother and Marco Tarantola. For more details about treatment and cell seeding please see [43].

The cardiomyocytes/fibroblasts ratio seeded on the different substrates is shown figure 2.1.

\subsubsection{Cell Staining}

The cardiomyocytes and fibroblasts seeded on $\mu$-Slides, kept under static conditions or under flow stimulation, were fixed and subsequently stained using immunostaining or fluorescent phallotoxins staining. The immunostaining was performed in order to visualize alpha-actinin localised in the Z-disks of cardiomyocytes, vimentin in $\mathrm{Fb}$, $\alpha$-smooth muscle actin ( $\alpha$-SMA) in myofibroblasts, and connexin-43/N-cadherin for electrical/mechanical cell-cell connections. Fluorescent phallotoxins was used to stain cell actin fibers. The procedure for cells fixation, permeabilisation and staining are described below. 


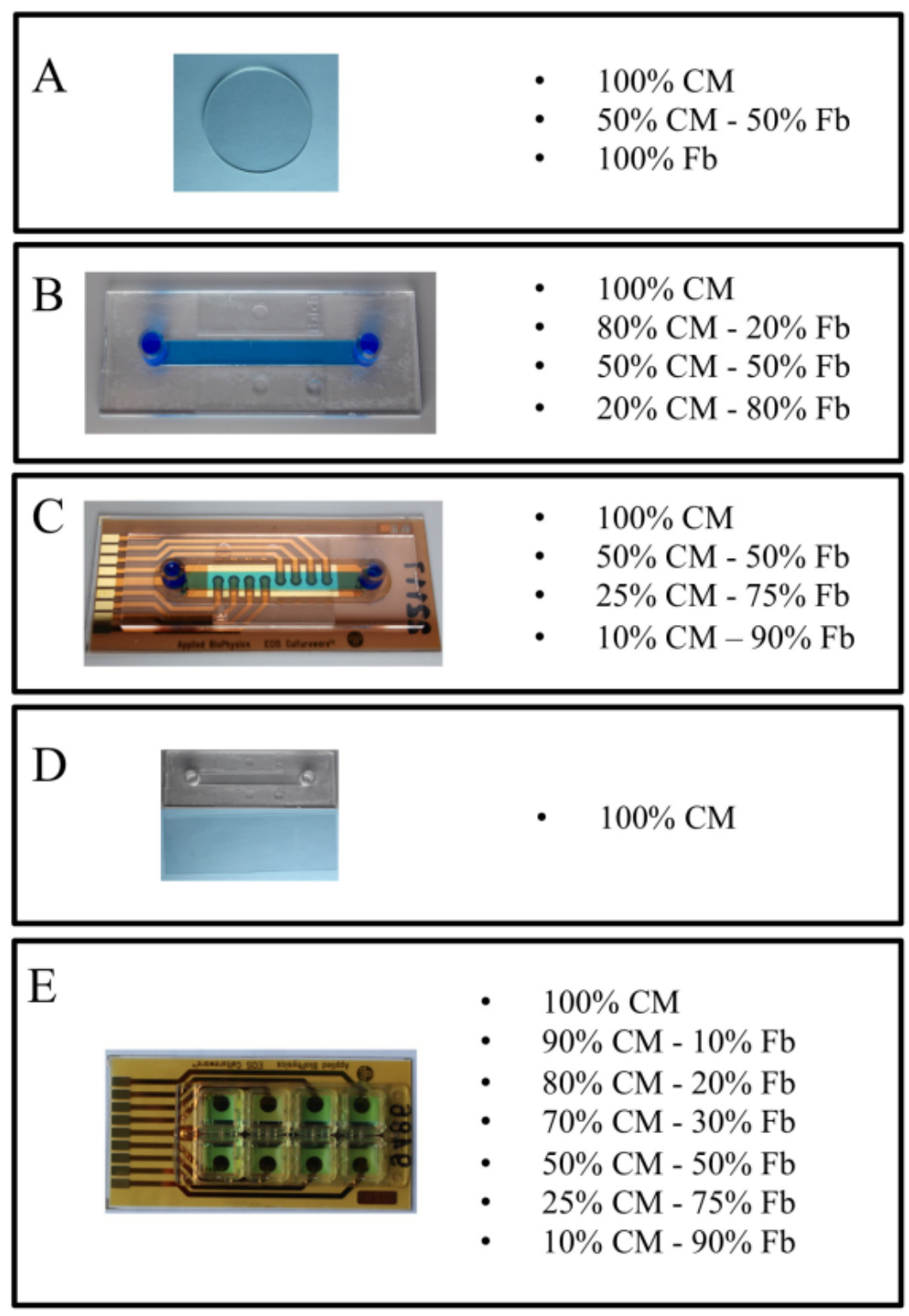

Figure 2.1: Substrates and the respective seeded cardiomyocytes (CM)/fibroblasts (Fb) ratio. A: Glass slides. B: $\mu$-Slide VI ${ }^{0.4}$ Luer, the channel was coloured in blue for the sake of visualisation. C: ECIS Flow Array 1E, the channel was coloured in blue for the sake of visualisation. D: sticky-Slide I ${ }^{0.4}$ Luer (upper part) and Glass Coverslips D 263 M Schott (lower part).E: ECIS 8W1E array.

\subsubsection{Fixation and Permeabilisation}

Before staining, cells were fixed by incubating $4 \%(\mathrm{w} / \mathrm{v})$ paraformaldehyle (PFA) in PBS for $15 \mathrm{~min}$ at RT. After washing twice with PBS, the cell membranes were permeabilised to enable dyes or antibodies to reach the intracellular space and stain the structures. Two different permeabilisation procedures were used for immuno- and fluorescent phallotoxins staining. The former was performed by adding $0.1 \%$ Triton-X-100 for $20 \mathrm{~min}$ at RT, then washing for $30 \mathrm{~min}$ at RT with a blocking buffer containing $5 \%$ FCS in $0.02 \%$ Tween 20. Later, PBS was added to block unspecific binding of the an- 
tibodies to non-target structures and reduce background staining. For the latter, $0.1 \%$ Triton-X-100 was added to the cells for 5 min at RT and then washed with PBS twice. Before adding the staining solution, the cells were pre-incubated for 30 min with PBS containing $1 \%$ bovine serum albumin (BSA).

\subsubsection{Immunostaining}

After blocking unspecific binding, cells were incubated overnight at $4^{\circ} \mathrm{C}$ with a primary antibody, which specifically recognises the target structures. After washing twice with PBS, a secondary fluorophore-labeled antibody, which binds to the primary antibody, was applied and stored for $45 \mathrm{~min}$ in the dark at RT. Afterwards cells were washed two times with PBS. For double or triple co-staining of different structures inside the cells, the procedure just described was repeated each time using secondary antibodies labeled with different fluorophores. The antibodies used in this work and their concentrations are listed in table 2.2. To easily distinguish cardiomyocytes from fibroblasts, the fluorophores on the secondary antibodies were chosen in a way to avoid spectral overlap. Cardiomyocytes were stained with anti- $\alpha$-actinin conjugated with DyeLight488 , fibroblasts with vimentin and $\alpha$-SMA conjugated with DyeLight-648, Connexin 43 and N-Cadherin with DyeLight-549. Finally the DNA/nuclei were stained by adding 4,6 diamidino-2-phenyindole (DAPI, Sigma-Aldrich, Seelze) at a concentration of $1 \mu \mathrm{g} / \mathrm{mL}$ to the cells for $20 \mathrm{~min}$ at RT and washed twice with PBS.

\begin{tabular}{lllcc}
\hline Antibody & Type & Species & Supplier & Dilution \\
\hline Anti- $\alpha$-actinin & primary & mouse & Sigma-Aldrich & $1: 150$ \\
Anti-vimenti & primary & mouse & Sigma-Aldrich & $1: 200$ \\
Anti- $\alpha$-SMA & primary & mouse & Sigma-Aldrich & $1: 400$ \\
Anti-N-Cadherin & primary & mouse & Sigma-Aldrich & $1: 100$ \\
Anti-Connexin 43 & primary & mouse & Sigma-Aldrich & $1: 150$ \\
DyeLight-488 & secondary & goat & Vector, Laboratories Inc. & $1: 150$ \\
DyeLight-549 & secondary & goat & Dianova, Jackson Immuno research Lab & $1: 150$ \\
DyeLight-648 & secondary & goat & Vector, Laboratories Inc. & $1: 150$ \\
\hline
\end{tabular}

Table 2.2: Antibodies used for immunostaining protocols

\subsubsection{Fluorescent Phallotoxin Staining}

The staining solution was obtained by diluting $5 \mu \mathrm{L}$ of fluorescent Alexa-Fluo-488phallotoxins (Invitrogen, UK) stock solution $(6.6 \mu \mathrm{M})$, previously prepared as suggested by the supplier, in $200 \mu \mathrm{L}$ of PBS and adding 1\% BSA, to reduce nonspecific background staining, for $30 \mathrm{~min}$ at RT. $300 \mu \mathrm{L}$ of staining solution was injected into $\mu$-Slide VI 0.4 with permeabilised cardiomyocytes. After washing twice with PBS the cell nuclei were stained as explained above. 


\subsection{Fluid Flow Stimulation}

To study the effect of the flow on the cells, a continuous laminar shear stress was applied to the monolayer. The setup and the experimental procedure are explained below.

\subsubsection{Physiological Shear Stress in vitro Estimation}

Under physiological conditions neonatal ventricular cardiac myocytes are subjected to a shear stress that can be estimated using the simple Couette flow model [9]. According to the model, the shear stress on cardiomyocyte monolayers is generated by the relative movement of myocardial laminar sheets (figure 2.2) and interstitial flow during each contraction. One of two parallel plates, separated by fluid with viscosity $\mu$ and by a distance $h$, is moving relative to the other with constant velocity $U_{0}$ generating a shear stress $\sigma$. Neglecting pressure gradients and considering a constant velocity, the Navier-Stokes equations can be simplified to

$$
\frac{\mathrm{d}^{2} u}{\mathrm{~d} y^{2}}=0
$$

where $y$ is a spatial coordinate perpendicular to the plates, $u(y)$ the velocity distribution and $u(0)=0$ and $u(h)=\mathrm{U}_{0}$ are the boundary conditions. Integrating twice with respect to $y$ the velocity profile is given by

$$
u(y)=\mathrm{U}_{0} \frac{y}{h}
$$

The shear stress generated is

$$
\sigma=\mu \frac{\mathrm{d} u(y)}{\mathrm{d} y}=\mu \frac{\mathrm{U}_{0}}{h}
$$

A laminar unit $a$ is defined as half of the height of each laminar sheet containing in between an interstitial gap $h$ filled a fluid of viscosity $\mu=3.5 \mathrm{cP}$ [44], as shown in figure 2.2. The shear strain is related to the height of the laminar unit $a$ and the laminar shear motion $b$ by the following formula

$$
\epsilon_{s}=\frac{1}{2} \epsilon_{s l}=\frac{1}{2} \tan (\theta)=\frac{1}{2} \frac{b}{a}
$$

where $\epsilon_{s l}$ is the engineering shear strain and $\theta$ the angle in between. By using literature values of the shear strain $\epsilon_{s}=0.15$ [45], the thickness of the cardiac laminae $(20 \mu \mathrm{m})$ 

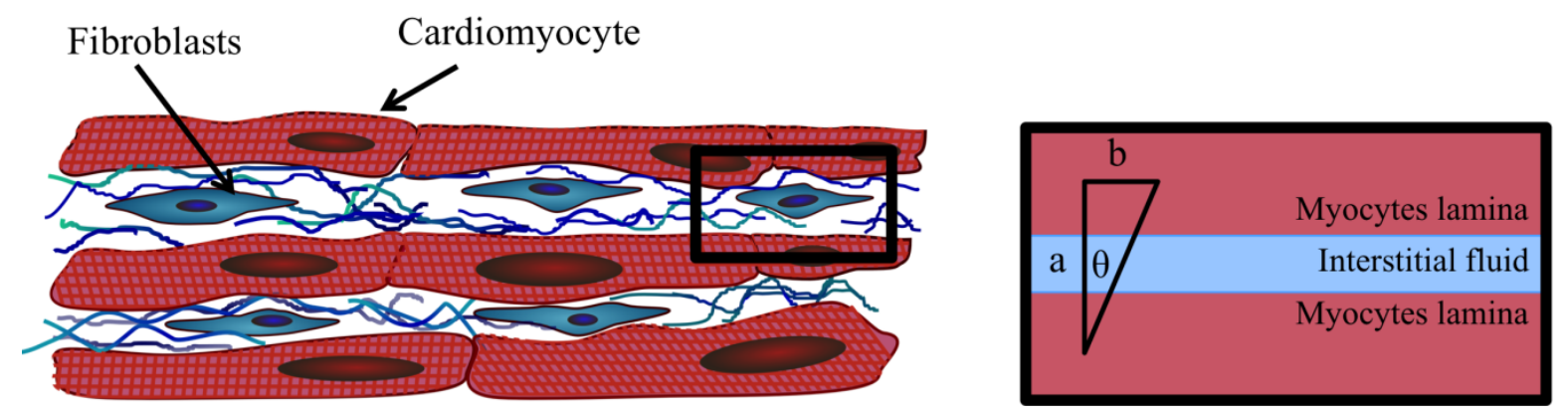

Figure 2.2: Scheme of myocardial sheets for shear stress calculation. Left: Schematic representation of myocardial laminar sheets separated by interstitial fluid. Right: Simplified illustration of interstitial fluid included between two cardiac lamina representing the Couette model used to calculate the shear stress on a defined laminar unit. The thickness of a laminar unit is $a$, that moves relatively to an other one with velocity $b / t$.

and interstitial gap $h=6.5 \mu \mathrm{m}$ [9] one can calculate the height of the laminar unit $a=1 / 2(20)+6.5+1 / 2(20)=26.5 \mu \mathrm{m}$. This can be used to extract the value of $b=$ $2 a \epsilon_{s}=7.95 \mu \mathrm{m}$. The plate velocity $U_{0}$ is obtained using the ratio between the laminar shear motion and the time $t$ during each contraction (systole)

$$
\mathrm{U}_{0}=\frac{b}{t}
$$

Assuming that the cardiac cycle is equally divided in systole and diastole, the systolic time for isolated neonatal cardiac myocytes whose beating frequency equal to $2.1 \mathrm{~Hz}$ [43], is $0.5 \mathrm{~s}$. Finally the shear stress is given by

$$
\sigma=0.17 \text { dyn } \mathrm{cm}^{2}
$$

\subsubsection{Setup}

Shear stress was applied to primary ventricular cardiac myocytes by an air-driven continuous flow pump system (Ibidi, Martinsried, Germany). This perfusion system consists of 3 parts as shown in the top of figure 2.3:

- Fluidic Unit (A) holds the gas permeable tubes and the fluidic reservoirs. It provides unidirectional constant flow by using two electrically controlled switch valves. The working principle is as follows: Air pressure is applied to one of the reservoirs filled with medium. This pushes the fluid in one direction while the pinch valves squeeze two of the four tubes inserted into the slots as shown in the scheme in figure 2.3. After a defined period of time, the air pressure is applied to the second reservoir, the squeeze tubes are released, and the valves pinch the other two tubes. In this way the direction of the flow in the the flow chamber is 

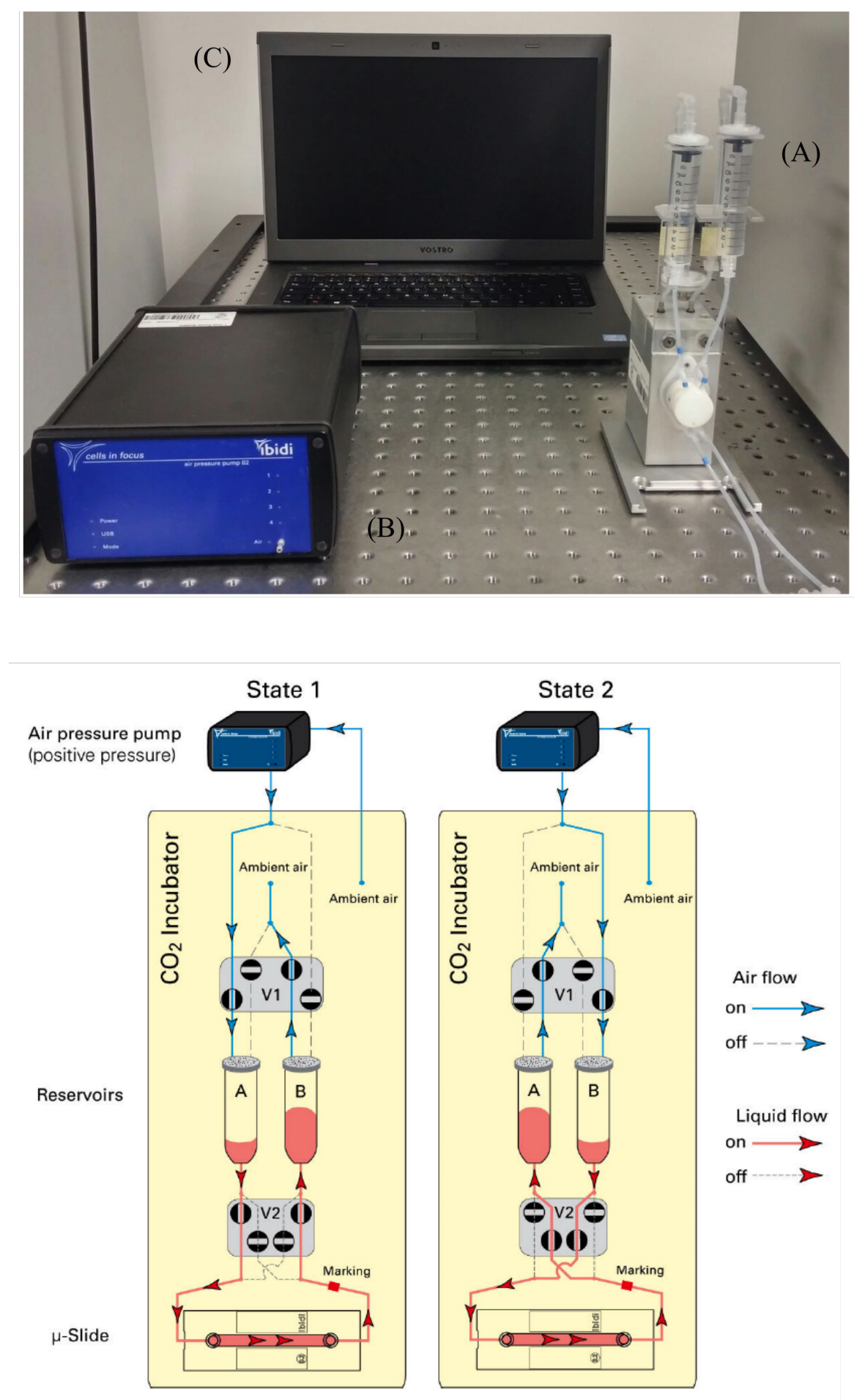

Figure 2.3: Perfusion set up.Top image Picture of single parts of the perfusion system: (A) fluidic unit, (B) air pressure pump, (C) Computer with pump controller software. Bottom image Scheme of the principles of flow generation (Ibidi Manual): air pressure is applied to one of the two reservoirs while the other is connected to ambient air pressure and flow is generated in the $\mu$-Slide channel (State 1). Switching valve V1, air is pumped in the reservoir B and clamping off two branches of the Perfusion Set, the medium is pumped back to reservoir A (State 2). This scheme was taken from the Ibidi Pump System Instruction, page 51. 
maintained. This allows one to apply a continuous laminar unidirectional shear stress for long time exchanging the fluid between the two reservoirs, avoiding the waste of medium. .

- Pump (B) controls the switching times of the electric valves and pumps air into the Fluidic Unit. This air is collected from the incubator in order to maintain the gas concentration inside the reservoirs. To protect the pump from the humidity coming from the incubator, the air is filtered using a drying bottle containing Silica beads.

- Pump controller $(\mathrm{C})$ is the user interface to set the experimental parameters.

\subsubsection{Experimental Procedure}

The cells were exposed to a continuous laminar flow for $24 \mathrm{~h}$ after seeding into a flow chamber, chosen according to the type of experiment. In order to investigate the effect of various shear stresses, tubes with different diameters were used for the experiment. Tubing, fluidic chambers and the values of the applied shear stresses are listed in table 2.3. Tubing and medium were left one night before starting the experiment at $37^{\circ} \mathrm{C}$ in $5 \% \mathrm{CO}_{2}$-humidified incubator before the experiment in order to equilibrate. Since the silicone tubes are permeable to gas and their permeability depends on the temperature, this process avoids air bubbles formation in the tubing due to rapid change from RT to $37^{\circ} \mathrm{C}$. This tubing was mounted on the Fluidic Unit under sterile conditions in a flow hood and filled with the medium. Any air bubbles present were removed from the tubes and then the substrates with cells were carefully connected to the perfusion system avoiding the entrapment of further bubbles. The Fluidic Unit was placed in incubator and kept inside for the entire experiment. In case this was not possible, for example while recording cells behaviour under a microscope, only the fluidic chamber with cells was kept at $37^{\circ} \mathrm{C}$ in $5 \% \mathrm{CO}_{2}$-humidified conditions. Before starting the flow the following parameters were set in the pump control software:

- shear stress required for the experiment

- type of tubing

- type of substrate used

- viscosity of the medium at $37^{\circ} \mathrm{C}\left(0.00072 \mathrm{dyn} \mathrm{s} / \mathrm{cm}^{2}\right)$

- valves switching time $(60 \mathrm{~s})$.

Cells were sheared continuously upto for $108 \mathrm{~h}$ and for $24 \mathrm{~h}, 48 \mathrm{~h}, 72 \mathrm{~h}$, according to the experimental procedure described in the section 2.4.1.1. The medium was exchanged daily and starting from the second day of stimulation medium "Day 0-2" was replaced by medium "Day 3". 


\begin{tabular}{ccc}
\hline $\begin{array}{c}\text { Shear Stress } \\
{\left[\text { dyn } / \text { cardiomyocytes }^{2}\right]}\end{array}$ & Tubes (Ibidi) & Substrate \\
\hline 1.59 & $\begin{array}{c}\text { Perfusion Set white (ID 0.8 mm, } \\
50 \text { cardiomyocytes) } \\
\text { Perfusion Set blue (ID 0.8 mm, } \\
15 \text { cardiomyocytes) }\end{array}$ & ECIS Flow Array 1E \\
& $\mu$-Slide VI ${ }^{0.4}$ Luer, \\
& Perfusion Set yellow-green (ID 1.6 mm, & ECIS Flow Array 1E \\
& 50 cardiomyocytes) & sticky-Slide ${ }^{0.4}$ Luer \\
& Perfusion Set blue (ID 0.8 mm, & \\
& 15 cardiomyocytes) & ECIS Flow Array 1E \\
\hline
\end{tabular}

Table 2.3: Values of shear stress applied to cells, type of tubes and substrates used in different experiments.

\subsection{Electric cell-substrate impedance sensing (ECIS ${ }^{\mathrm{TM}}$ )}

ECIS is a non-invasive quantitative technique to characterise morphological changes and cell passive biological properties of adherent cellular monolayers [46]. The complex impedance $Z$ of the cell monolayer is measured by applying an alternating current $(1 \mu \mathrm{A})$ at varying frequencies between a small working electrode and a larger counter electrode. The electrical connection between the electrodes is provided by the cell culture medium above the insulating cell layer. The frequency dependent impedance of the cell monolayer provides information about cell-cell connectivity, cell-substrate distance and membrane properties. At low frequencies the current is forced to flow underneath the cells and in the intercellular space, at high frequencies, it can pass through the cells.

In this work, ECIS was used to investigate the effect of high shear stress on morphological and functional changes of cardiac cell monolayers over the time as well as, the effect of shear stress on cardiomyocytes/fibroblasts co-cultures contractility.

\subsubsection{Cellular Impedance Modelling}

The monolayer-electrode-medium system can be described by an equivalent circuit as shown in figure 2.4A. By using this simple physical model [47] one can distinguish the effect of different cellular properties on the overall impedance. The contribution of a cell-free electrode covered by electrolyte (cell medium) is modelled with a constant phase element $(\mathrm{CPE})$ in series with an Ohmic resistor $R_{\text {bulk }}$. The impedance spectra as a function of frequency for a cell-free and an cell-covered electrode are shown in figure 2.4D. When cells adhere onto the electrode, the impedance of the system increases due to the frequency-dependent contribution $Z_{c}$ by the cellular layers. At low frequencies, the impedance $Z_{c}$ is dominated by the capacitance of the cell membrane $C_{m}$ and therefore the current is forced to flow underneath the cells and in the intracellular space. Whereas, 

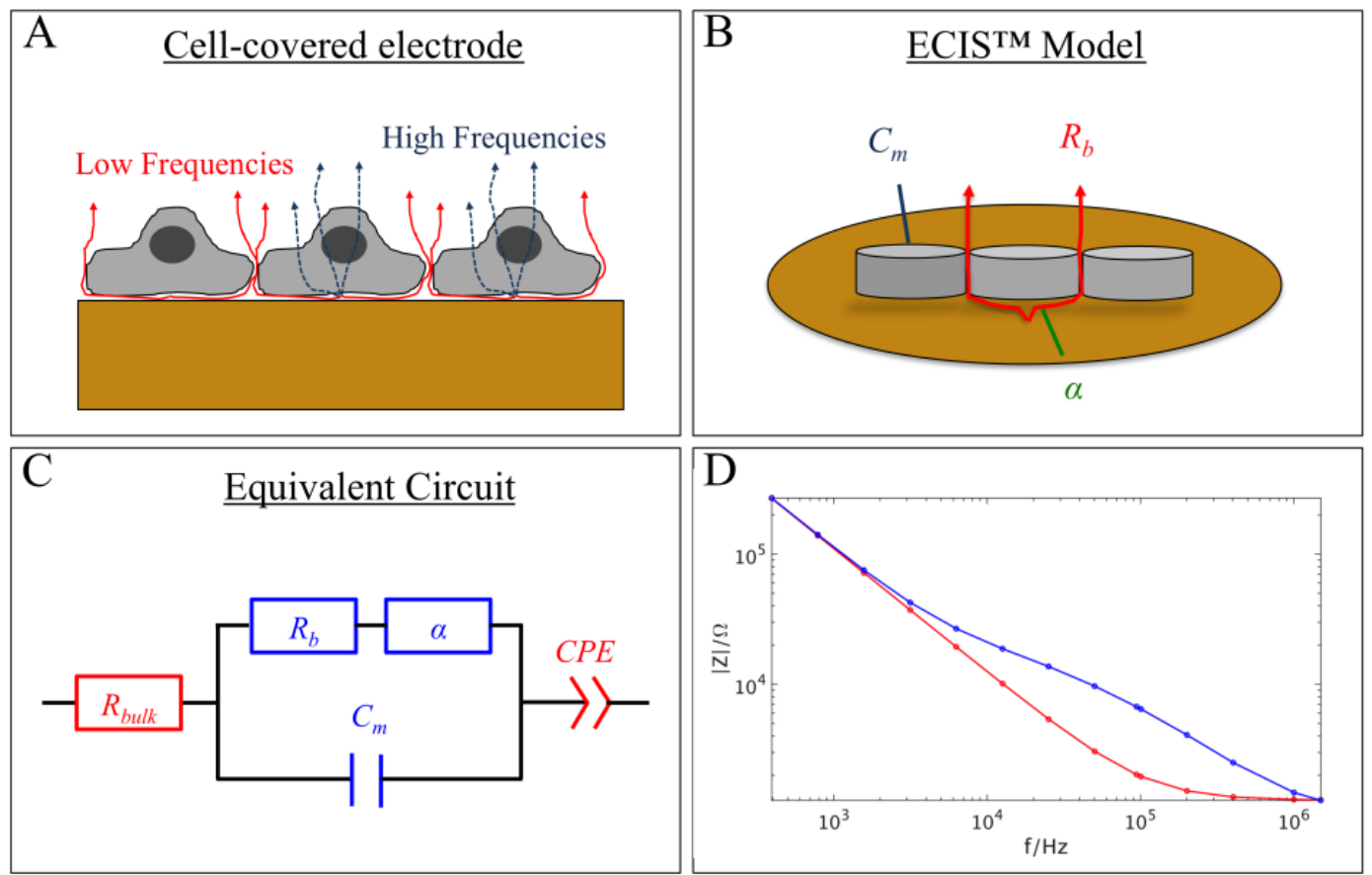

Figure 2.4: Working principles of ECIS. A: Schematic illustration for a cell-covered gold electrode. The cell layer frequency- dependent impedance is given by two spatially different current pathways: at lower frequencies the current flows underneath the basal cellular membrane and through the intercellular gaps between cells $[\rightarrow]$, at high frequencies the current passes through the cell $[\rightarrow]$. At intermediate frequencies the current uses both pathways with different ratios. B: Schematic representation of the model developed by Giaever and Keese (1991) and modified by Lo et al. The cells shape is assumed to be a rectangle with two half disks at the extremities. Frequency- dependent impedance contributions results from three parameters. $R_{b}$, describing the intercellular current path and quantifying cell-cell connectivity. $\alpha$ accounts for the impedance rising from the cell-substrate cleft and depends on the cellelectrode distance. $C_{m}$ is the capacitative contribution of the cellular membrane. C: Equivalent circuit model for cell-covered electrode defined by the impedance contribution of the naked electrode (red) and of the adherent cells (blue). The cell-free electrode is described by a constant phase element (CPE) in series with the resistance of the medium $R_{b u l k}$. D: Impedance spectra as function of frequency for cell-free (red) and cell-covered (blue) electrode.

at high frequencies, $C_{m}$ is shortcutted and $Z_{c}$ arises from two resistive elements: $R_{b}$ (barrier resistance) measure of the cell-cell contact density, and $\alpha$ that is proportional the cell-substrate distance. In this case, the current can pass through the cell. The model that was used to calculate the cell-covered specific impedance $\mathrm{Z}_{c}$ (impedance per unit area) as a function of frequency, assumes the cell shape to be rectangular with half disk on each end [48]. The frequency dependent $Z_{c}$ is given by:

$$
\begin{aligned}
Z_{c}=\left(Z_{n}+Z_{m}\right)[1 & +\left(\frac{L W}{L W+\frac{\pi}{4} \omega}\right)\left(\frac{\frac{2 Z_{m}}{Z_{n}}}{\gamma W \operatorname{coth}\left(\frac{\gamma W}{2}\right)+2 R_{b}^{r e c}\left(\frac{1}{Z_{n}}+\frac{1}{Z_{m}}\right)}\right) \\
& \left.+\left(\frac{\frac{\pi W^{2}}{4}}{L W+\frac{\pi W^{2}}{4}}\right)\left(\frac{\frac{2 Z_{m}}{Z_{n}}}{\frac{\gamma W}{2} \frac{I_{0}(\gamma W / 2)}{I_{1}(\gamma W / 2)}+2 R_{b}^{d i s k}\left(\frac{1}{Z_{n}}+\frac{1}{Z_{m}}\right)}\right)\right],
\end{aligned}
$$




$$
\gamma=\left[\frac{\rho}{h}\left(\frac{1}{Z_{n}}+\frac{1}{Z_{m}}\right)\right]^{\frac{1}{2}}
$$

where $W$ is the cell width, $L$ is the cell length and $\rho$ is resistivity of the bulk cell culture medium. $I_{0}$ and $I_{1}$ are modified Bessel function of the first kind of order 0 and $1 . Z_{n}$ represents the specific impedance of the cell-free electrode modeled by CPE, that is a non-ideal capacitor, characterised by two adjustable parameters $n$ and $A$. $Z_{n}$ behaves like an ideal resistor when $n=0$ and like an ideal capacitor with a capacitance equal to $A$, whereas it shows both resistive and capacitive behaviour for values of $n$ between 0 and 1.

$$
Z_{n}=\frac{1}{(i \omega)^{n} A}
$$

$Z_{m}$ describes the specific membrane impedance of the cells with $C_{m}$ the average capacitance of both apical and basal cell membrane:

$$
Z_{m}=\frac{1}{i \omega C_{m}}
$$

The cell-covered specific impedance depends on two parameters: $\alpha$, related to the average height between basal cell membrane and the substrate,

$$
\alpha=\frac{\gamma W}{2}\left(\frac{1}{Z_{n}}+\frac{1}{Z_{m}}\right)^{-\frac{1}{2}}=\frac{1}{2} W\left(\frac{\rho}{h}\right)^{1 / 2}
$$

and the barrier resistance between cells $R_{b}$, obtained from the contribution of the current passing through the two geometrically separated cell areas (rectangular and disk)

$$
R_{b}^{r e c}=\frac{\frac{4 L}{W}+2 \pi}{\frac{4 L}{W}+\pi} R_{b}
$$

and

$$
R_{b}^{d i s k}=\frac{\frac{2 L}{W}+\pi}{\frac{4 L}{W}+\pi} R_{b}
$$

By fitting the equation 2.7 to the experimental data using least-square algorithm, the model parameters $R_{b}, C_{m}$ and $\alpha$ were quantified. 


\subsubsection{Setup and Experimental procedure}

The cellular monolayer impedance was measured with an ECIS setup Z $\Theta$ (Applied Biophysics, Troy, NY,USA) and a Flow Array 1E electrode chamber. The eight measuring electrodes with diameter of $250 \mu \mathrm{m}$, equally spaced along the $50 \mathrm{~mm}$ flow channel, were coated and seeded with cells, as explained previously in section 2.1.1. The chambers were connected to the perfusion system, placed in a holder, and kept at $37^{\circ} \mathrm{C}$ in $5 \% \mathrm{CO}_{2^{-}}$ humidified conditions. In order to detect defects in the array, test measurements were performed for few minutes with single frequency/time mode (SFT) at $4 \mathrm{KHz}$. This frequency provides the best signal to noise ratio. The functional or morphological changes in cellular monolayers under the effect of shear stress were monitored by collecting the time course impedance in two different modes.

\section{Monitoring cellular contractile motion}

Fast impedance fluctuations due to the spontaneous beating of cardiomyocytes were measured in rapid time collect (RTC) mode for 2 min at $4 \mathrm{kHz}$ with a sampling frequency of $28 \mathrm{~Hz}$. Data was recorded every $24 \mathrm{~h}$ before the medium was exchange. This avoid measurement artifacts due to temperature jumps while opening the incubator door or flow interruptions.

\section{Monitoring Cellular morphological changes}

In order to study the response of the cells upon shear stress, the impedance of cell covered electrodes was measured with multiple frequency/time (MFT) at eleven frequencies ranging from 62.5 to $64000 \mathrm{~Hz}(62.5 \mathrm{~Hz}, 125 \mathrm{~Hz}, 250 \mathrm{~Hz}, 500 \mathrm{~Hz}, 1000 \mathrm{~Hz}, 2000 \mathrm{~Hz}$, $4000 \mathrm{~Hz}, 8000 \mathrm{~Hz}, 16000 \mathrm{~Hz} 32000 \mathrm{~Hz}, 64000 \mathrm{~Hz}$ ). The time resolution of the MFT mode was $\sim 2.6$ min for a sequential measurement of sixteen electrodes (two flow arrays with 8-electrodes each). The measured impedance values at the 11 frequencies were used to obtain $R_{b}, C_{m}$ and $\alpha$.

The impedance of the cell monolayer was continuously measured in MFT-mode for a duration of $24 \mathrm{~h}$ until $108 \mathrm{~h}$ to allow a daily cell medium exchange. The shear stress was applied approximately $30 \mathrm{~min}-40 \mathrm{~min}$ after the beginning of the measurement on the first day. Before exchanging the medium, measurements in MFT-mode were interrupted and the contractile motion of the cells was recorded. Afterwards, the fluid stimulation was paused to allow the medium exchange and then the MFT impedance monitoring was restarted.

\subsubsection{Impedance Analysis}

The measured data were analysed by different methods to study the effect of shear stress on cells contractility (functionality) or on cell-cell/cell-substrate junctions and 
cell shape (morphology).

\section{Contractile motion analysis}

Spontaneous cells beating frequency was obtained from the real part of the cell monolayer complex impedance, recorded with RTC-mode, using Fast Fourier transformation (FFT). One can use the FFT to derive the power spectral density (PSD) which contains the amplitudes of the frequencies the signal is composed of. Since cardiac cells, seeded on measuring electrodes, contract with a certain beating rate, the current arising from underneath the basal membrane consequently change its amplitude due to the movement, generating periodic fluctuations in the complex impedance. By performing the PSD analysis on the recorded real part of the impedance the beating frequency was extracted from the position of the first peak. If the cells were not beating, no clear peak was detected in the PSD, indicating no periodicity in the signal. Statistical significance was determined by Wilcoxon Rank Test or t-test. Differences were considered significant at $P<0.05$.

\section{Analysis of cellular morphological changes}

In order to analyse the dynamics of the short-term response of the cell to the flow onset, the real part of the mean of the time-course impedance at $4 \mathrm{kHz}$ was normalised to the data collected before the flow onset. Within a defined temporal window of $3 \mathrm{~h},\left(t_{0}-0.5\right.$, $\left.t_{0}+2.5\right)$, where $t_{0}$ is the the time of the flow onset, $Z_{\text {Renorm } @ 4 k H z}$ was fitted, using a custom-written MATLAB program, with a combination of exponential and linear rise terms, as given below

$$
Z_{\text {Renorm } @ 4 k H z}= \begin{cases}c-a e^{-\frac{(t-t 0)}{\tau}}+b\left(t-t_{0}\right), & \text { if } t>t_{0} \\ c-a, & \text { if } t \leq t_{0}\end{cases}
$$

where $t_{0}$ and $\tau$ are fitting parameters and $a, b, c$ linear coefficients. $t_{0}$ and $\tau$ which represents the time constant of the exponential rise, are obtained by minimising the least-square error between the fitted and experimental data. The errors of the parameters were determined with a Bootstrapping approach. The exponential rise mimics the immediate and fast cell response to the shear stress onset, while the linear term models the later and slower response. $\tau$, corresponding to the time $Z_{\text {Renorm } @ 4 k H z}$ needs to reach $\sim 63 \%$ of the exponential fit asymptote, was used to describe the dynamics of the response of the system to the applied shear stress.

To obtain the ECIS parameters $R_{b}, C_{m}, \alpha$, the experimental data were compared to the model described in section 2.3.1 using a fitting algorithm based on least square 
analysis. The determined $R_{b}, C_{m}$ and $\alpha$ values from the impedance spectra at a time point were used as initial guess values for the subsequent time point. In this way, the parameters were obtained as functions of time. The resistance of the bulk cell medium which is not included in $Z_{c}$ in equation 2.7, was defined by the constriction resistance $\frac{\rho \pi r^{2}}{4 r}$, with $\rho=54 \Omega \mathrm{cm}, r=125 \mu \mathrm{m}$. Additionally, we used $C_{n}=12 \mu \mathrm{Fcm}^{-2}$, and $n=0.95$ for modeling the impedance of the naked electrode. Cell size was measured from confocal micrographs by Dr. Claudia Richter (Max Planck Institute for Dynamics and Self-Organization, Göttingen, Germany): $W=22 \mu \mathrm{m}, L=46 \mu \mathrm{m}$ [43]. Statistical significance was determined by Wilcoxon Rank Test or T-test to compare values of the model parameters at different shear stress. Differences were considered significant at $P$ values less than $0.05(* P<0.05, * * P<0.01, * * * P<0.001)$

\subsection{Microscopy}

In this work, confocal laser scanning microscopy (CLSM) was used to quantify cellular connectivity under different fibrotic conditions and to evaluate fiber orientation due to stimulation with shear stress extended over time, as will be explained in section 2.4.1. Atomic force microscopy (AFM) was employed to characterise cardiac fibrosis rheological properties 2.4.2. Reflection interference contrast microscopy (RICM) 2.4.3, was used to determine the effect of shear stress on cell-substrate contact area and cellsubstrate distance optically, and to correlate these observations with the impedance spectroscopy results. All the microscopes and objectives used are listed in table 2.4.

\begin{tabular}{|c|c|c|}
\hline Microscope & Microscopy & $\begin{array}{l}\text { Objectives } \\
\text { (type, magnification, } \\
\text { aperture) }\end{array}$ \\
\hline $\begin{array}{c}\text { FluoView1000 } \\
\text { (Olympus, Tokyo, Japan) }\end{array}$ & CLSM & $60 \times / 1.35$ Oil \\
\hline $\begin{array}{c}\text { MFP-3D } \mathrm{D}^{\mathrm{TM}} \\
\text { (Asylum Research, Santa Barbara, CA, USA) }\end{array}$ & AFM & \\
\hline $\begin{array}{c}\text { IX } 51 \\
\text { (Olympus, Tokyo, Japan) }\end{array}$ & Phase contrast & $60 \times / 0.75$ \\
\hline $\begin{array}{c}\text { IX } 83 \\
\text { (Olympus, Tokyo, Japan) }\end{array}$ & RICM & UApoN $100 \times / 1.4 /$ Oil \\
\hline
\end{tabular}

Table 2.4: Microscopes, specifications and objective used. 


\subsubsection{Confocal Laser Scanning Microscopy}

Confocal laser scanning microscopy (CLSM) produces sharp focused images by scanning a focused excitation beam through the fluorescently labeled sample. The out of focus blur due to the contribution of the light from specimen above and below the focal plane is rejected with the help of a pinhole. CLSM has a lateral resolution on the order of $\lambda / 2$ which allows one to record small details within a thin section of the sample with high contrast. The focused excitation beam is scanned across the sample with the help of multiple mirrors to generate a 2D-image. Acquiring a series of $2 \mathrm{D}$ images at different positions along the z-axis by scanning the sample along the z-axis, the three-dimensional structure of the sample can be imaged. In this work, CLSM was mainly employed to image 2D sections of fixed cultures of cardiomyocytes-fibroblasts mixtures after immunostaining and shear stress stimulated cardiomyocytes cultures after phalloidin staining.

\subsubsection{Experimental Procedure}

2D micrographs of cardiac myocytes and fibroblasts co-cultures with different fibroblast fractions $(0 \%, 20 \%, 50 \%, 80 \%)$ were acquired using a CLSM based on an inverted microscope in order to quantify cellular connectivity under fibrotic conditions. The cells seeded on $\mu$-Slides were cultivated in static conditions for 5 days and fourfold stained as explained in section 2.1.3.2. Images from eight different regions along the $50 \mathrm{~mm}$ channel were acquired on an Olympus FluoView 1000 microscope (Olympus GmbH, Germany) using a $60 \mathrm{X}$ oil, N.A. 1.35 objective (Olympus, Germany). The excitation was performed using a sequential line scanning mode. Lasers of wavelength $488 \mathrm{~nm}$ and $649 \mathrm{~nm}$ were used together first, followed by the excitation using the wavelengths $549 \mathrm{~nm}$ and $405 \mathrm{~nm}$ (DAPI) in order to image the cellular structures labeled with four colors as described in section 2.1.3.2. In this way, the cross talk between the spectral channels was minimized. A pinhole of size $100 \mu \mathrm{m}$ was used to reject the out of plane fluorescence. The fluorescence was recorded in a region of interest of $512 \times 512$ pixels in a camera that contains $1024 \times 1024$ pixels. These experimental parameters were kept constant for all the experiments.

In order to study the time course of actin fibers orientation under shear stress, 2Dimages of cells stained with Alexa-Fluor-488-phallotoxins were recorded. The sheared cells and controls were fixed and stained (section 2.1.3.3) $24 \mathrm{~h}, 48 \mathrm{~h}, 72 \mathrm{~h}, 108 \mathrm{~h}$ after the onset of the flow. In this case, an excitation wavelength of $488 \mathrm{~nm}$ and a camera with $1024 \times 1024$ pixels were used. 


\subsubsection{Quantification of cellular connectivity}

The cell-cell connectivity via junction proteins was analyzed on the images acquired using the CLSM with a custom written Python (www.python.org) program. First, we count the number of cells of each type in the image in order to match with the ratio of the designed co-culture. Only those images that show the number of cells close to the set ratio were used for the connectivity analysis. The program was written by Fabian Knoch (Max Planck Institute for Dynamics and Self-Organization, Göttingen, Germany). The schematic representation of the working principle of the program is shown in figure 2.5. Let us give three different colors to the visualized items. Cardiomyocytes appear green, fibroblasts red and the junction proteins connexin 43/N-cadherin appear yellow. The program detects yellow pixels that share a border with red/green or both red and green pixels and creates a binary matrix. After binarization, yellow pixels are identified and the algorithm counts the number of neighbouring red and green pixels. The counting is done upto $N$ nearest neighbour pixels. This parameter depends on the pixel size and the imaging resolution. For our discussion, let us consider $N=1$, as shown in the figure. Yellow pixels close to the edge of the image (edge- $N$ ) are neglected. Each yellow

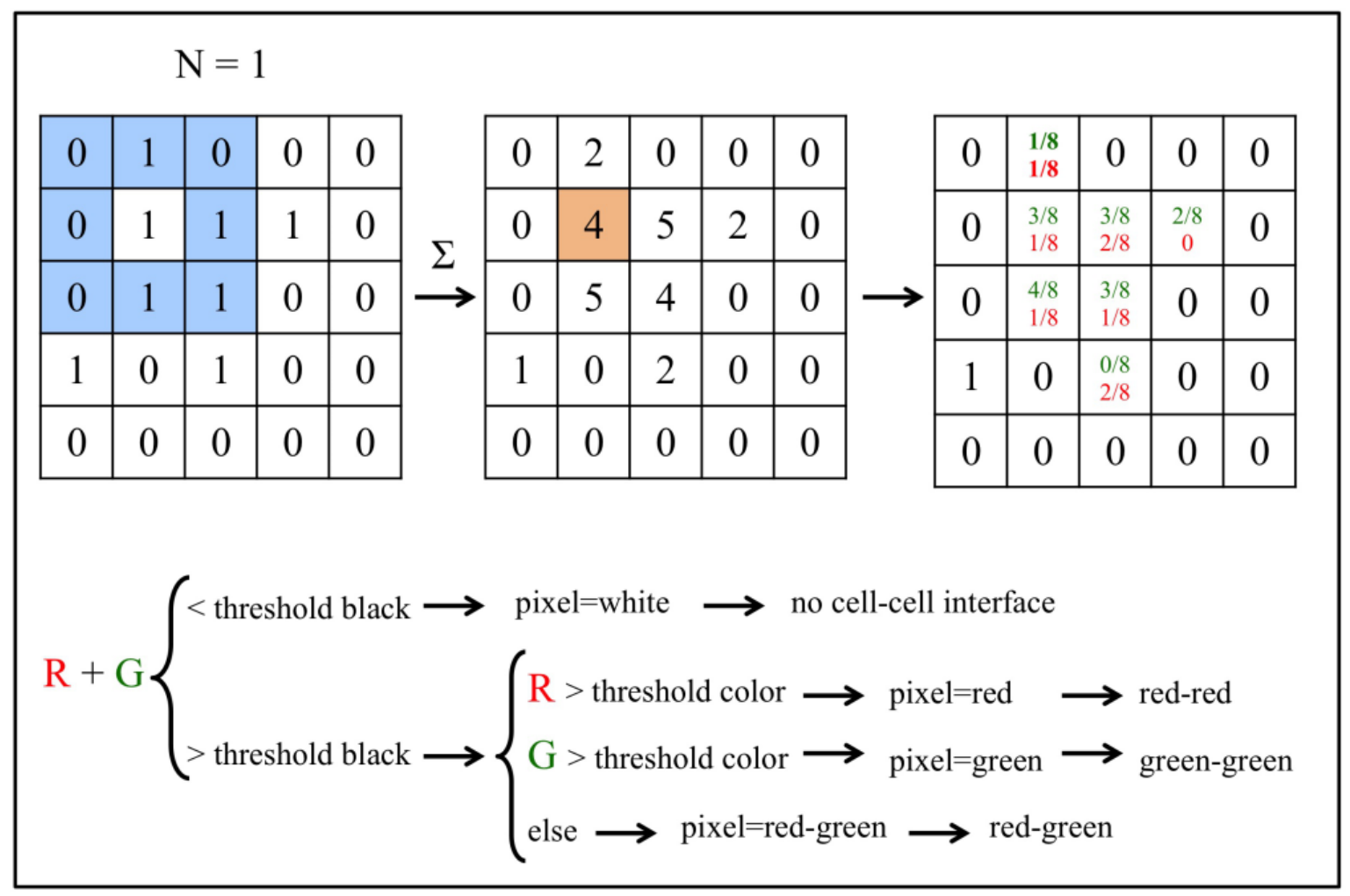

Figure 2.5: Algorithm for cell connectivity quantification. Schematic illustration to detect green (cardiomyocytes), or red (fibroblasts), or both surrounding a yellow pixel (representative of cell junction proteins). $\mathrm{N}$ is the number of neighbouring pixels analysed per single yellow pixel. The sum of green and red surrounding pixels is compared first with a background threshold. Pixels with values higher than the background threshold are compared with a specific color threshold. 
pixel is assigned two values: one representing the number of neighbouring red pixels, and the second giving the number of neighbouring green pixels divided by the total number of neighbouring pixels. Finally the algorithm calculates the sum of both green and red values and compares it to a background threshold parameter. If the sum is lower, the yellow pixel will be set to zero. This means that there are not enough red or green pixels next to the yellow ones. In this way, single noisy pixels and unspecifically bound dye are excluded. If the sum is larger, the green and red value is individually compared with a colour threshold parameter. The yellow pixel is considered in between two cardiomyocytes if the green value is larger than the color threshold, and similarly in between two fibroblasts if the red value is larger. If no individual value (red or green) is bigger than the threshold, the yellow pixels is considered between cardiomyocyte and a fibroblast. For the analysis of the connectivity between cardiomyocytes and fibroblasts. In these experiments, $N$ was set to 5 .

\subsubsection{Analysis of fibers orientation}

Image analysis of actin fibers was performed in close collaboration with Dr. Narain Karedla (Third Institute of Physics-Biophysics, Georg-August-University, Göttingen, Germany), who implemented the fiber orientation analyser program. Fluorescence intensity images of labeled actin fibers obtained from the CLSM are read into MATLAB. Figure 2.6(A) shows an exemplary image of raw data as acquired from the microscope, without any further modifications. The distribution of the orientation of the labeled fibers can be done conveniently using a Fourier transform approach. The linearity and rotation properties of the Fourier transform (FT) were used. Linearity means that the FT of a superimposition of multiple images is identical to the summation of FTs of individual images. The rotation property implies the fact that when the image is rotated by an angle $\theta$, the FT in the Fourier space gets rotated by the same angle. The FT of a line will have components in directions orthogonal to it. In this way, the FT is composed by the orientation information corresponding to all the measured fibers. Studying the angular distribution of the components in the FT directly gives the required orientation distribution. Restricting the discussion to square images (equal rows and columns), the orientation quantification is done by using the following steps:

1. Padding: The raw data, typically of size $1024 \times 1024$, were placed at the center of a matrix of twice its dimensions $(2048 \times 2048$ pixels $)$. This matrix was padded with the mean intensity of the raw data.

2. Windowing: This padded image was then multiplied with a modified 4-term Blackman-Harris window which is equal to unity in the center $1024 \times 1024$ pixels [49]. The rows and columns of the padded image are multiplied by this function giving rise to a windowed image as shown in Figure 2.6(B). Windowing is done 

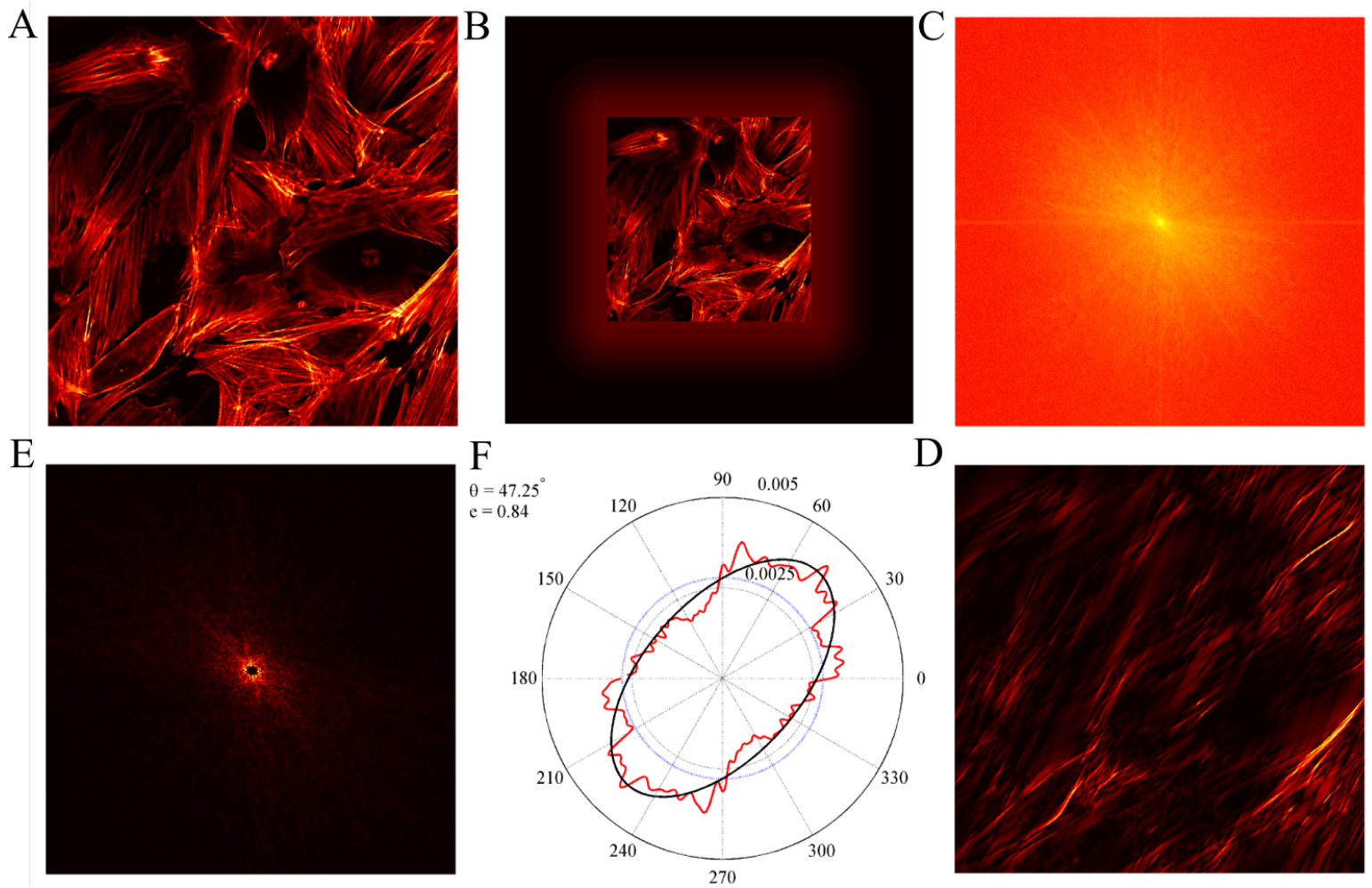

Figure 2.6: Image analysis for determining the orientation distribution of the labeled actin fibers. (a) exemplary image; (b) windowed image using a 4-term Blackman-Harris window; (c) 2D-FFT image of (b); (d) Butterworth filter applied to the Fourier image (c); (e) polar plot showing the normalized fiber orientation distribution in (a) fitted with an ellipse; and (f) back calculated image showing fibers around the direction of the orientation maximum. For reference, a blue dotted circle representing the probabilty for completely random orientation of fibers is shown in (e).

generally to reduce spectral leakage, which is an intrinsic problem while using discrete/fast Fourier transform algorithms [50]. The window used here does not effect the intensity information in the raw data as can be seen from the figure. Following this, the FT is computed, and the image 2.6(C) shows the 2D-plot of the absolute values.

3. Radial Band-Pass Filtering: The center of the FT image contains low frequency information due to background fluctuations in the image and the finite image size used for the analysis. The high frequency components are dominated by shot noise and edges of the image. In order to remove such noise and undesired high- and low-frequency components, the FT was weighted with a fourth-order Butterworth low-pass and sixth-order Butterworth high-pass filters, respectively. The net filter can be written as:

$$
F\left(f_{r}\right)=\frac{1-\beta f_{r}}{\left.\left(1+\left(f_{L} / f_{r}\right)^{12}\right]\left[1+\left(f_{r} / f_{H}\right)^{8}\right]\right)^{1 / 2}}
$$

where $f_{r}$ is the normalized radial frequency, $f_{L}$ and $f_{H}$ are the low-pass and high- 
pass cutoff frequencies, respectively, and $\beta$ is a constant that controls the slope of the filter function at intermediate frequencies. It is absolutely flat for $\beta=0$. In these experiments, $\beta=0.7$ was used, as reported earlier [49]. Figure 2.7 shows the amplitudes of the radial band-pass filter used for the applications. The FT is multiplied with this radial filter function to obtain the weighted amplitudes as shown in Figure 2.6(D).

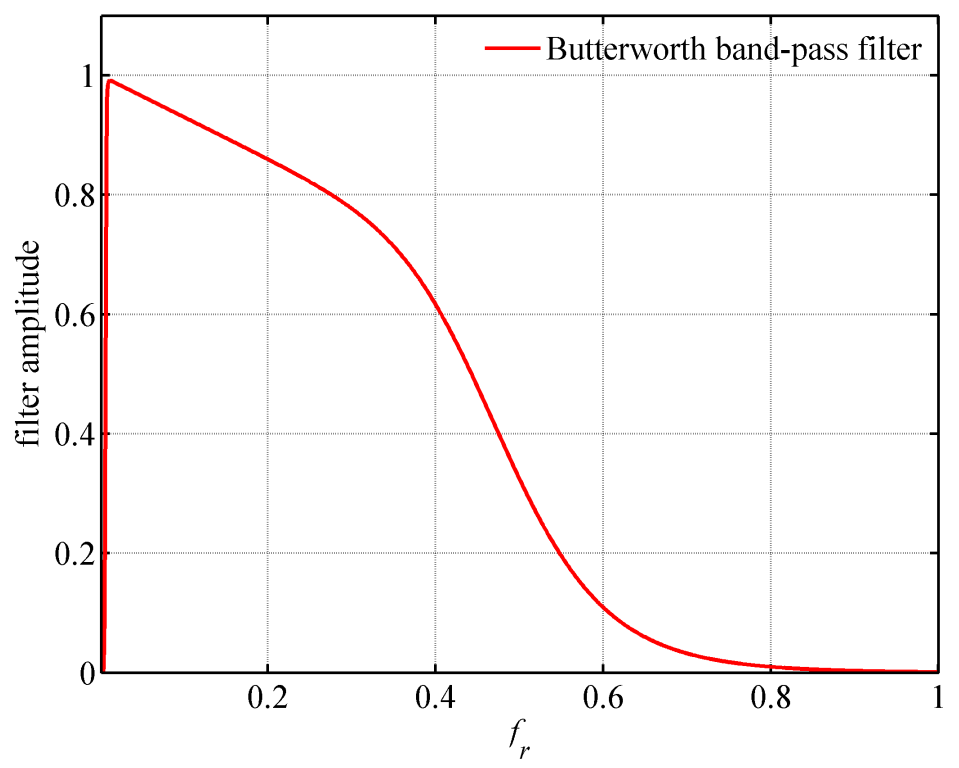

Figure 2.7: Radial amplitude of a band-pass filter constituting a fourth-order Butterworth low-pass and sixth-order Butterworth high-pass filters together, represented by equation 2.15 . The values $f_{L}$ and $f_{H}$ were set to 0.002 and 0.5 , respectively.

4. Directional Integration: The amplitudes of the weighted FT are summed for sectors with widths of $2^{\circ}$ to $5^{\circ}$ individually for various orientations of center angle from the y-axis (FT components along the y-axis represent the fibers oriented along the x-axis, and so on). A polar plot of the normalized distribution of the summed amplitudes for the central angles is shown in Figure 2.6(E). This shows the normalized probability distribution of the orientation of the fibers in the raw image.

5. Ellipse Fitting: A completely random orientations of fibers will show up as a circular distribution as shown in Figure 2.6(E) by the blue-dotted circle. Therefore, any directed orientation of the fibers will lead to a symmetric bimodal distribution with elongation parallel to the net orientation. In order to determine the orientation of the elongation and quantify the orientation factor, the distributions were fitted with an ellipse as shown in the same figure. The rationale behind fitting an ellipse is that it is equivalent to resolving the second order principal moments (or components of a 2D-gyration tensor) for the symmetric bimodal distributions that 
will be obtained during such orientation analysis. The eccentricity $e$ of the fitted ellipse $\left(\sqrt{1-(b / a)^{2}}\right)$ can be used as a quantity to estimate the orientation factor, where $a$ and $b$ are the major and minor radii, respectively. For a completely random distribution of orientations, $e=0$, and for an ideal case of perfectly oriented fibers $e \approx 1$. The direction of the major axis is the direction of orientation $\theta_{\max }$. The eccentricity characterizes the orientation factor. It can, for example, be used to get an estimate of the half-angle $\theta_{\text {err }}$ from $\theta_{\max }$ within which $67 \%$ of the fibers are oriented using the following equation

$$
\theta_{\text {err }}=\tan ^{-1}\left[\sqrt{1-e^{2}} \tan \left(\frac{\pi A_{\text {err }}}{2}\right)\right]
$$

where $A_{\text {err }}$ is the fraction of the fibers (in this case it is 0.67 ). Figure 2.8 shows the calculated $\theta_{\text {err }}$. For $e=0$ the $\theta_{\text {err }}$ is $60^{\circ}$ (for a circular distribution, this is easy to understand since the area of two opposite sectors of $120^{\circ}$ contribute to $2 / 3$ the total area of the circle), and as $e$ increases, the $\theta_{\text {err }}$ goes to $0^{\circ}$. In this work, it was considered that an image shows well oriented fibers only if $e \geq 0.9$, which corresponds to a $\theta_{\text {err }} \leq 37^{\circ}$.

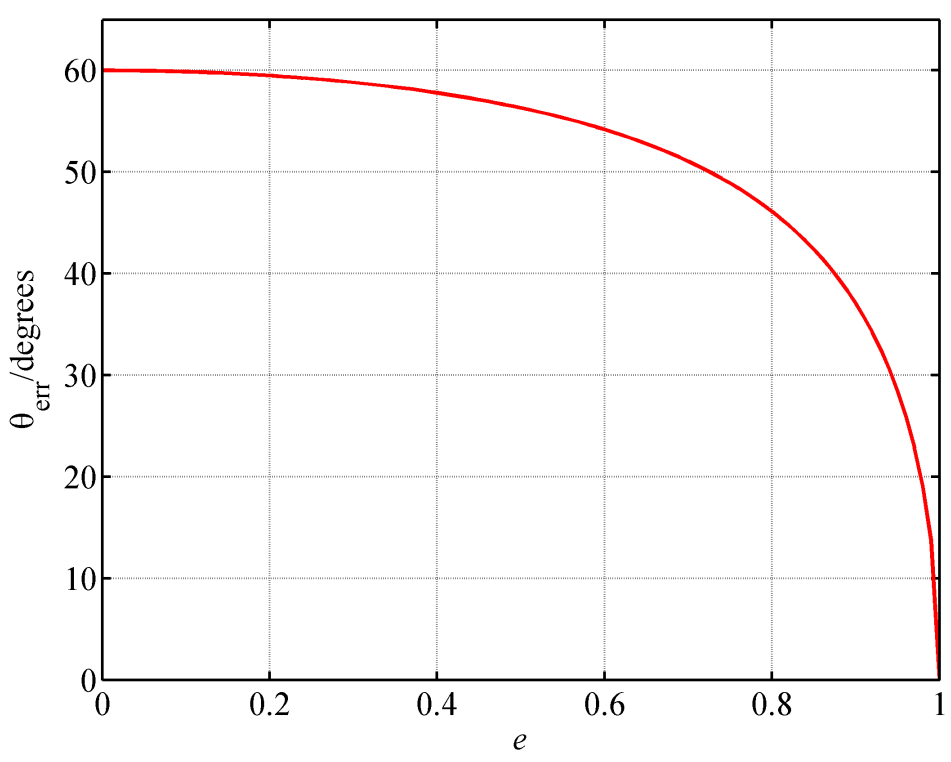

Figure 2.8: Plot of $\theta_{\mathrm{err}}$ for various $e$ at $A_{\mathrm{err}}=0.67$.

\subsubsection{Atomic force microscopy-based microrheology}

Atomic force microscopy is a scanning probe technique extensively used for high resolution imaging and characterising the mechanical properties of the cells. The specimen is scanned in the $\mathrm{x}-\mathrm{y}$ directions with the help of a piezo stage while the vertical position of a nanometer probe tip attached to a flexible cantilever is controlled by a another 
z-piezoelectric element. A laser beam is reflected from the head of the cantilever onto a quadrant photodiode (QD). The deflection of the cantilever is measured by monitoring the deflection of this reflected laser beam on the QD. QD signal is also used as a feedback for determining the force or the height of the cantilever as shown in figure 2.9. The sample is scanned either by holding the height of the z-piezoelectric element as a constant or by using applying a constant force by controlling the deflection of the cantilever.

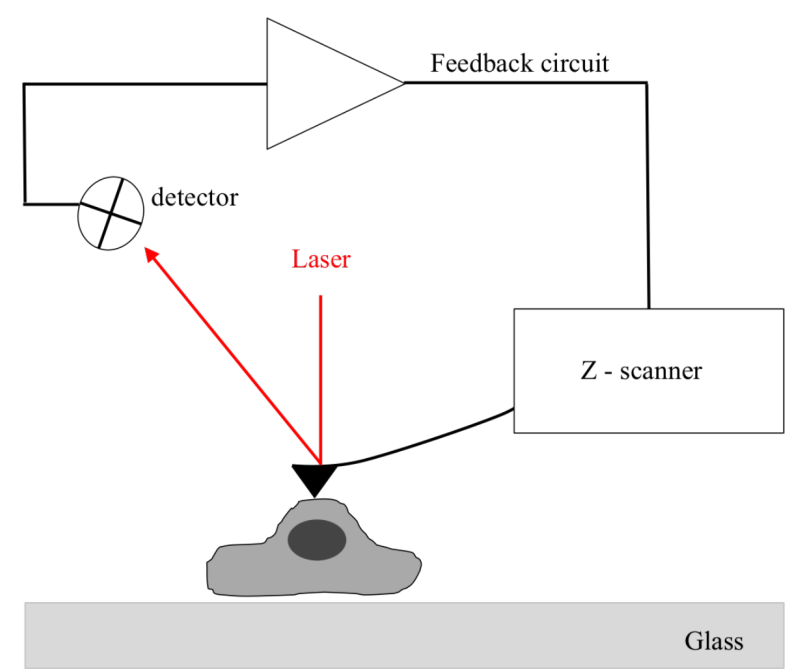

Figure 2.9: Schematic drawing of AFM principle. The deflection of the cantilever induces changes in the direction of the reflected laser beam and it is used by the position-sensitive photodetector (PSD) to control, with a feedback circuit, the height of the probe.

The loading force $F$ exerted by the cantilever is proportional to the spring constant $k_{c}$, the deflection $x$ and is given by the Hooke's law $F=k_{c} \cdot x . k_{c}$ is measured by monitoring the thermal fluctuations using the relation $k_{c} \approx k_{B} T /<x^{2}>$ (the proportionality constant takes the geometry and the non-ideal behavior of the cantilever into account). The elastic properties of the cells are obtained from the relation between $F$ and the indentation depth $\delta$ of the tip into the cell surface. The indentation depth $\delta$ can be calculated from the net displacement of the z-piezoelectric element by subtracting the deflection $x$. Due to the viscoelastic nature of the cells, they are able to store and dissipate mechanical energy and their response to mechanical perturbations depends on the speed at which the stimulus is applied. To study these microrheological characteristics, Shroff and co-workers [51, 52] developed a technique whereby low amplitude sinusoidal oscillations are applied after the cantilever tip indents the cell surface as shown in figure 2.10 .

The relation between force and indentation of a four-side pyramidal indenter ([53]), 


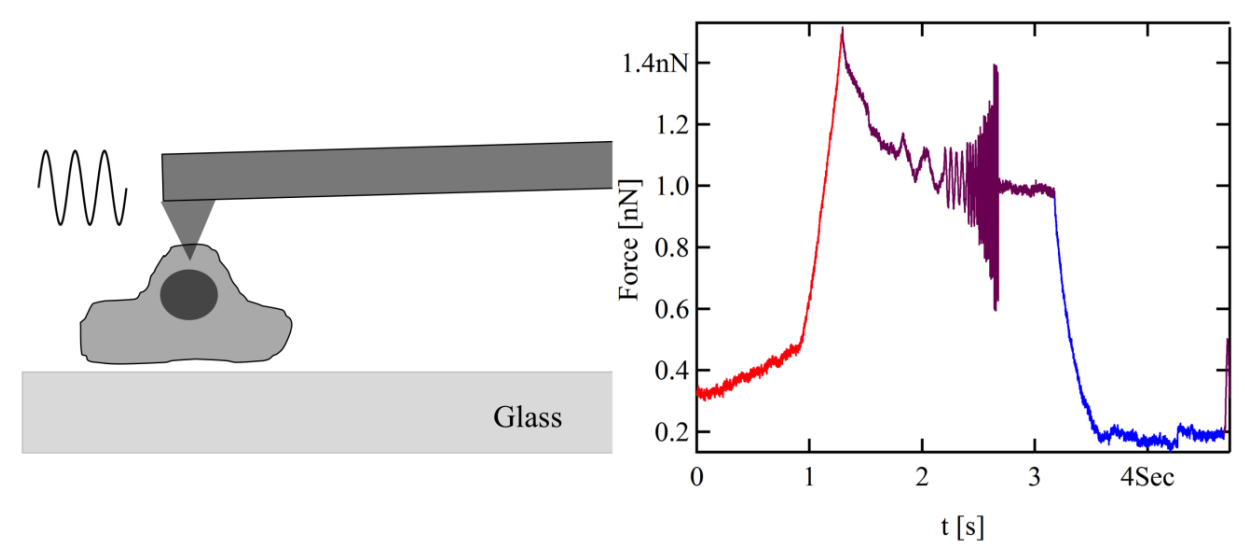

Figure 2.10: Schematic drawing of the AFM-based microrheology. The cantilever, once in contact with the cell surface oscillates with small amplitude at different frequencies (left). Time course of force during measurements: force increases sharply when the probe is in contact with the cell. Afterwards the cantilever is made to oscillate at various frequencies (from low to high) with predefined amplitudes. In the end, the force amplitude decreases when the cantilever withdraws from the cell surface. (right).

according to Hertz model, is

$$
F=\frac{3 E \tan \theta}{4\left(1-\nu^{2}\right)} \delta^{2}
$$

where $E$ and $\nu$ are the elastic modulus and the Poisson's ratio of the cell, respectively. $\theta$ is the half opening angle of the indenter. By neglecting the higher order terms from the Taylor expansion and plugging in the relation $G=E / 2(1+\nu)[52]$ in the above equation, the complex shear modulus $G^{*}$ of the cell can be approximated with an expression in the frequency domain, written as

$$
G^{*}(\omega)=\frac{1-\nu}{3 \delta_{0} \tan \theta} \frac{F(\omega)}{\delta(\omega)}
$$

where $F(\omega)$ and $\delta(\omega)$ represent the Fourier transform of $F$ and $\delta$ at the angular frequency $\omega$, and $\delta_{0}$ is the initial indentation. Since the cantilever is subjected, both, to the force applied to the cell and to the viscous friction due to its movement in the surrounding liquid, the equation 2.18 can be corrected to yield

$$
G^{*}(\omega)=\frac{1-\nu}{3 \delta_{0} \tan \theta}\left[\frac{F(\omega}{\delta(\omega)}-i \omega b(0)\right]
$$

where $i \omega b(0)$ represents the hydrodynamic drag forces and $b(0)$ is estimated from noncontact measurements [54]. The complex shear modulus is the summation of the storage modulus $G^{\prime}$ and the loss modulus $G^{\prime \prime}$, accounting for stored elastic energy and dissipated energy, respectively $\left(G^{*}(\omega)=G^{\prime}(\omega)+i G^{\prime \prime}(\omega)\right)$. The ratio of $G^{\prime \prime}$ and $G^{\prime}$ is defined as loss tangent $\eta$, which represents the tangent of the phase shift between the force and 
indentation. It is an index of the viscoelastic behaviour of the cells: for $\eta<<1$ they show a solid-like behaviour, and for $\eta>>1$ they behave as viscous liquids.

\subsubsection{Setup and Experimental Procedure}

AFM-based rheological experiments were performed with a MFP-3D ${ }^{T M}$ (Asylum Research, Santa Barbara, CA, USA), combined with a BioHeater ${ }^{T M}$ and placed on an inverted microscope (Olympus IX 51). A C-microlever on a MLCT cantilever (Bruker, Camarillo, USA) with spring constant $10 \mathrm{pN} / \mathrm{nm}$ was used for both force measurements and imaging. First, the QD was calibrated by establishing the linear relation between the photodiode current measured and the piezo z position. This was done when the cantilever was in touch with an incompressible cell free glass surface. Second, the spring constant of the cantilever was determined by measuring the thermal fluctuations. Lastly, the hydrodynamic drag forces were estimated by oscillating the cantilever at various heights $(200 \mathrm{~nm}, 400 \mathrm{~nm}, 600 \mathrm{~nm}, 800 \mathrm{~nm}, 1 \mu \mathrm{m}, 1.5 \mu \mathrm{m}, 2 \mu \mathrm{m}, 2.5 \mu$, $3 \mu \mathrm{m}$, and $3.5 \mu \mathrm{m}$ )(figure 2.11A). Calibration, hydrodynamic drag force records and force measurements were performed at $37^{\circ} \mathrm{C}$ (samples where pre-heated about $1 \mathrm{~h}$ before to avoid fluctuations in cantilever deflections due to shifts of temperature) and with HEPES free cell medium. Afterwards, the bare glass was replaced with samples seeded with cardiomyocytes and/or fibroblasts, cultivated for 5 days, as previously explained in chapter 2.1.1. Force maps and cell rheological properties were measured while the cantilever was oscillating around a given indentation depth with frequencies between $5 \mathrm{~Hz}$ and $150 \mathrm{~Hz}(5 \mathrm{~Hz}, 20 \mathrm{~Hz}, 40 \mathrm{~Hz}, 60 \mathrm{~Hz}, 80 \mathrm{~Hz}, 100 \mathrm{~Hz}$, and $150 \mathrm{~Hz})$ at small oscillation amplitudes $(20-50 \mathrm{~nm})$ using a built-in mode in the Asylum Research MFP software ([55]). For each force map (20 point $\times 20$ point grid), 400 force-distance curves were recorded for the area of interest. Thereafter, Blebbistatin $(4 \mu \mathrm{M})$ was added to cell culture with cardiomyocytes to arrest their contraction in order to acquire a height image of the same area with contact mode. Force measurements were performed on multiple areas on the same sample after the medium was exchanged.

\subsubsection{Extraction of microrheological parameters}

Data were processed using a MATLAB program ShearFM.m ([55]) written by Dr. Jan Rother (Institute of Physical Chemistry, Georg-August-University, Göttingen, Germany). In this program one can select the position within the measured force map for analysing the force indentation curves. Once the data have been loaded, both excitationforce response phase shift and the hydrodynamic drag transfer function, defined as $H_{D}(\omega)=F_{D}(\omega) / \delta^{\prime}(\omega)$, were corrected. $F_{D}, \delta^{\prime}$ and $\omega$ stand for the Fourier transforms of the drag, the deflection, and the frequency of oscillation, respectively. First, the experimental mean deviation between the excitation-force response phase shift and the 
theoretical shift for a Newtonian viscous fluid $\left(90^{\circ}\right)$ were fitted linearly and the results subtracted from the difference of force oscillation and indentation depth $\left(\varphi_{F}-\varphi_{\delta}\right)$ (figure 2.11B). After phase shift correction, experimental $H_{D}$ measured at different tip-glass distances were used to estimate $b(0)$, computing $b(h)$ with $H_{D}=2 \pi \omega i b(h)$ (hydrodynamic drag correction) (figure 2.11C).

A

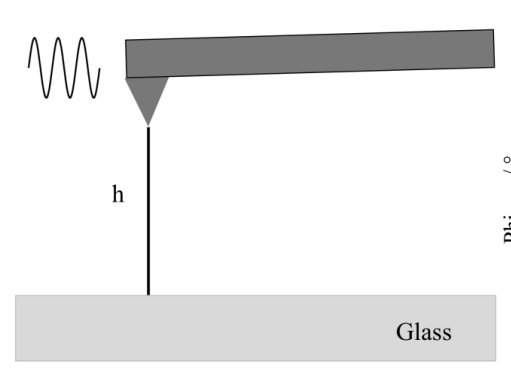

B

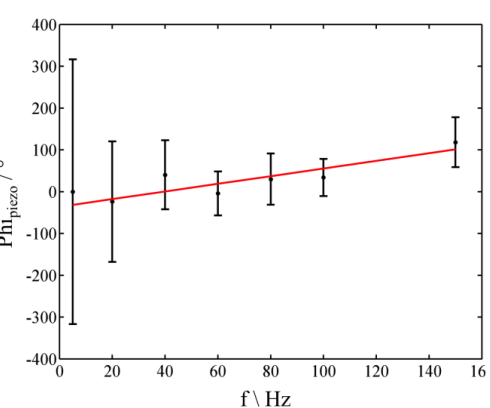

$\mathrm{C}$

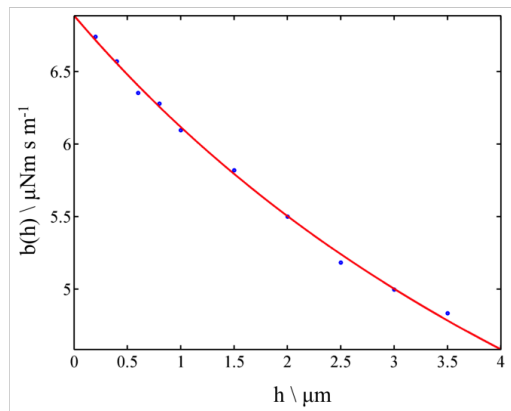

Figure 2.11: Schematic drawing of the hydrodynamic drag correction. (A)The cantilever is exited with sinusoidal oscillations with frequencies from $5 \mathrm{~Hz}$ to $150 \mathrm{~Hz}$ at different tip-glass distances.(B) Example of deviation from $90^{\circ}$ phase shift between excitation-force response over frequency. (C) Drag coefficient $b(h)$ fit as function of tip-sample distance for extrapolation of $b(0)$

The force-indentation curves of the selected positions in the force map were analysed to extract storage modulus $G^{\prime}$, loss modulus $G^{\prime \prime}$ and loss tangent $\eta$. In order to analyse only the data measured on cells and avoid artifacts introduced by scanning the bare glass substrate the positions were chosen overlapping each force map with the respective AFM image. The calculated complex shear modulus $G^{*}$ was fitted with the power-law structural damping model [52], interpreted as a particular case of soft glassy rheology. In the model cells are treated as an active soft glassy material and the cellular cytoskeleton as a network of structural elements that weakly interact and that are not permanently attached to each other. Each element is trapped in energetic wells and needs to jump out of the wells in order to deform. This occur when the system is perturbed by an external force or there is an active motion induced by motor proteins. As result the cell shift to either more fluid-like state, where the element are able to jump more between energy wells, or to solid-like state, characterised by a reduced level of element agitation. The power-law coefficient $\beta$ describes how freely the elements of the cytoskeleton can move: $\beta=0$ is indicative of a completely elastic solid-like material and $\beta=0.5$ of a fluid-like state. The equation for the fit with the power-law structural damping model is

$$
G^{*}(\omega)=G_{0}\left(1+i \tan \left(\frac{\alpha \pi}{2}\right)\left(\frac{\omega}{\omega_{0}}\right)^{\beta}+i \omega \mu\right.
$$

where $\beta$ is the power-law coefficient, $\mu$ is the Newtonian viscosity of the cell, $G_{0}$ and 
$\omega_{0}$ are scaling factors for stiffness and frequency, respectively. There parameters were obtained from a non-linear least squares fitting algorithm.

\subsubsection{Reflection Interference Contrast Microscopy}

Reflection Interference Contrast Microscopy (RICM) is an optical microscopy technique that allows one to measure the proximity of an object, for example a cell, to a glass surface with a nanometer resolution. It uses the reflected light from glass/medium and medium/cell membrane interfaces to create a 2-dimensional matrix of intensities. The light, focused on the sample, is partially reflected at each interface because of the different refractive indices. At the medium/cell membrane interface the phase of the reflected light is shifted compared to the phase of the wave reflected from the glass/medium interface, causing interferences and resulting in a pattern of bright/dark pixels in the recorded image. The closer the cell is to the glass surface, the larger is the phase shift of the medium/cell membrane reflected light, therefore larger interference occurs and the pixels appear darker compared with the ones where the cell is further away for the glass. In RICM, the intensity of the signal is a qualitative measure of the height of the cell from the substrate. Due to its high axial resolution $\sim 5 \mathrm{~nm}$, label free nature, and the possibility of combining it with fluorescence microscopy, RICM is highly suitable for studying the dynamics of adhesion in soft systems [56].

In this work, RICM was used to optically study the short-term effect of shear stress on cardiomyocytes, especially to monitor the changes in cell-substrate distance and cell contact area.

\subsubsection{Setup and Experimental Procedure}

RICM setup was installed on an inverted microscope equipped with a 100X oil immersion objective (NA 1.4, UApoN, Olympus) with a built-in lambda-quarter plate. The light from a halogen lamp was filtered at $\lambda=560 \mathrm{~nm}$ (MT-20 Light Source/Filter Wheel, Olympus), then linearly polarized, reflected and circularly polarized by a polarizer, semi-reflecting mirror and the lambda-quarter plate, respectively. After reflection at the sample interface, the light is converted back to linear polarized and imaged by a CCD camera (ORCA-Flash4.0, Hamamatsu). The microscope was enclosed in a custombuilt heating chamber (PeCon $\mathrm{GmbH}$, Germany) and the sample was placed into a $\mathrm{CO}_{2}$ controlled humidified frame inside the heating chamber. In order to investigate cell adhesion dynamics under shear stress stimulation, cardiomyocytes were seeded on sticky-Slide I ${ }^{0.4}$ Luer flow channel combined with a glass coverslip, as explained in section 2.1.2. The channel was connected to the flow system and placed under the microscope, preheated at least $2 \mathrm{~h}$ before in order to minimise temperature-induced 
focus drift. A sequence of images was acquired for $24 \mathrm{~h}$ every $5 \mathrm{~min}$ with an exposure time of $363 \mathrm{~ms}$. After 6 to 8 images (30 min - $40 \mathrm{~min}$ ) the shear stress was applied. In order to ensure that the cell's immediate response to the shear stimulation is recorded, the flow was turned on just a few seconds before an image was captured. Cardiomyocytes functionality, i.e., beating frequency, was verified after $24 \mathrm{~h}$ of flow stimulation.

\subsubsection{Analysis of intensity and contact-area}

Image analysis was performed to determine, both, the cell-substrate contact area and its intensity distribution over the time in a time window of $3 \mathrm{~h}$ ( $30 \mathrm{~min}$ before and $2.5 \mathrm{~h}$ after the shear onset). Every $15 \mathrm{~min}$ in the defined time window, frames were selected manually and analysed. In order to study the long-term effect of the shear stress, the contact area evaluation was extended to $20 \mathrm{~h}$ and the images were selected $30 \mathrm{~min}$ before and $30 \mathrm{~min}, 1.5 \mathrm{~h}, 2.5 \mathrm{~h}, 3 \mathrm{~h}, 5.5 \mathrm{~h} 10.5 \mathrm{~h}, 15.5 \mathrm{~h}$, and $19 \mathrm{~h}$ after the shear onset. From each selected frame the boundary of the cells was defined manually. This was done because the common cell segmentation or thresholding tools were found to be ineffective to estimate the cell boundaries in a crowded co-culture. Cells whose parts of the projected area disappeared from the field of view at any time during the time window, were discarded from the analysis. Projected cell area (in $\mu \mathrm{m}$ ) and intensity histogram within the defined cell boundary were measured for each frame. The intensity histogram was evaluated to obtain a qualitative estimate of the cell-substrate distance over time when shear stress was applied. The brightness histogram is sensitive to experimental artifacts, such as defocusing. In order to correct for brightness changes that are not related to the cell-substrate distance variations, the brightest pixels in the sequence of images were scaled to the brightest pixel of the frame recorded just before the flow was applied. This was done using a custom written MATLAB program. By using this brightness correction, the intensity changes of the pixels corresponding to the cell indicate the effective cell-substrate distance variation over time. 


\section{$3 \quad$ Results}

\subsection{Mechanoelectrical Coupling and Mechanics in a Cardiac Fibrosis Model}

This section presents the characterisation of electrical and mechanical coupling between the cardiomyocytes and fibroblasts within an in vitro cellular model mimicking different stages of fibrosis progression. An increasingly severe cardiac fibrosis can be reproduced by varying the ratio of fibroblasts and cardiomyocytes in a co-culture. Mutual cell interactions were analysed by direct observation of junction proteins in cardiomyocytefibroblasts cardiomyocytes-cardiomyocites and fibroblasts-fibroblasts samples via immunostaining. Changes in the functional electro-mechanical coupling upon increasing the level of fibrosis was both observed optically using propagation-induced phasecontrast (PIPC) [43] and quantified using ECIS that will be presented in this chapter. Furthermore the viscoelastic properties of cardiac cells are presented in this section. AFM-based microrheology was employed to examine the viscoelastic properties of coculture of cardiomyocytes and fibroblasts (1:1 ratio) co-culture, which was used as a fibrotic model. These viscoelastic properties were compared with the properties of the monocultures.

\subsubsection{Coupling via Cellular Junction Proteins}

Parts of this subchapter and some figures have been published in the following journal article:

Rother, J.; Richter, C.; Turco, L.; Knoch, F.; Mey, I.; Luther, S.; Janshoff, A.; Bodenschatz, E.; Tarantola, M. "Crosstalk of cardiomyocytes and fibroblasts in cocultures" Open Biology 5150038 (2015) 


\subsubsection{Electrical Junctions}

Co-cultures of cardiomyocytes and fibroblasts were seeded, cultivated for 5 days, stained via immunohistochemistry as described in section 2.1). The goal of these experiments was to quantify the spatial distribution of the heterocellular and homocellular junctions. The electrical gap junctions between two cells were visualised by the occurrence of connexin 43 (Cx43), whereas neural plate-cadherin (N-Cad) was indicative for adhesion junctions. The figure 3.1 shows the results of the electrical junction protein quantification. On the top of the figure, the quantification of the $\mathrm{Cx} 43$ in the co-cultures with various ratios is given. The bottom part of the figure shows four magnified confocal images, where the cardiomycytes are shown in red, the fibroblasts in green, the Cx43 proteins in yellow or white and the nuclei in blue. The staining of gap junction protein in cultures with $100 \%$ cardiomyocytes culture revealed that $85 \pm 2 \%$ of the total amount of $\mathrm{Cx} 43$ localised between the cardiomyocytes, $12 \pm 4 \%$ at the border between cardiomyocytes-fibroblasts and $3 \pm 1 \%$ between fibroblasts-fibroblasts. The presence of fibroblasts and few endothelial cells within the cardiomyocytes monoculture cannot be avoided and is due to the standard procedure for isolation of primary cardiac cells. However, during the experiment the amount of non-cardiomyocytes was considered negligible. Between cardiomyocytes in monocultures the stained Cx43 is shown in bottom left micrograph of figure 3.1. In co-cultures with cardiomyocytes-fibroblasts with 1:1 ratio, $55 \pm 10 \%, 13 \pm 3 \%, 34 \pm 9 \%$ of junctional $\mathrm{Cx} 43$ was localized in regions between cardiomyocytes-cardiomyocytes, cardiomyocytes-fibroblasts, and fibroblasts-fibroblasts, respectively. The two top micrographs in figure 3.1 show the heterocellular contacts between cardiomyocytes-fibroblasts in the co-culture mentioned above. The left one is a merged image of $\alpha$-actinin in cardiomyocytes, fibroblasts, and nuclei. The right image shows the $\mathrm{Cx} 43$ staining alone for the same image. When the amount of cardiomyocytes in the co-culture is reduced to 20\%, most of the Cx43 was expressed between fibroblasts and fibroblasts $(72 \pm 5 \%)$. The $\mathrm{Cx} 43$ staining in this co-culture is shown in the bottom-right micrograph in figure 3.1. The distribution of the gap junction between cardiomyocytes-fibroblasts and cardiomyocytes-cardiomyocyte in the same coculture was around $15 \pm 2 \%$ and $13 \pm 5 \%$. These results lead to the conclusion that Cx43 junctions are present between all possible pairs in co-cultures cultivated at different cadiomyocytes and fibroblasts ratio. Overall, the amount of homocellular junctions depends on the percentage of fibroblasts in co-cultures, whereas the heterocellular junctions remained unchanged.

\subsubsection{Mechanical Junctions}

The occurrence of mechanical adhesion junctions in cardiomyocytes monoculture and in cardiomyocytes-fibroblasts co-culture was quantified by staining N-Cad protein. The 


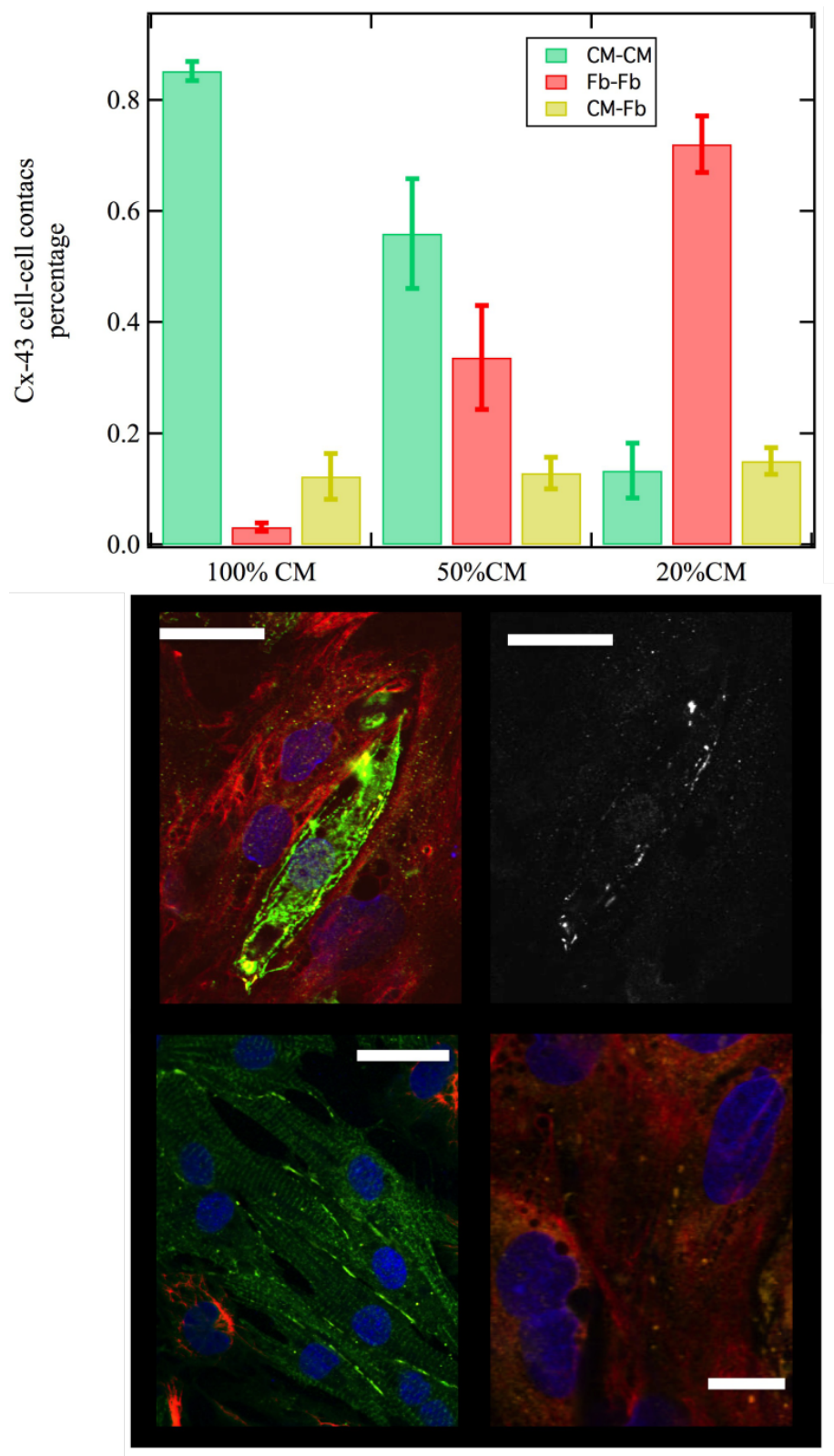

Figure 3.1: Electrical junctions Top graph: Distribution of heterocellular and homocellular electrical coupling between cells in co-cultures with different cardiomyocyte(CM)-fibroblast (Fb) ratios. $\mathrm{Cx} 43$, indicative of electrical gap junctions was localized at the border between fibroblasts, cardiomyocytes and cardiomyocytes-fibroblasts. The amount of protein between homocellular electrical junctions cardiomyocytes-cardiomyocytes and fibroblasts-fibroblasts raised with respectively the increase of cardiomyocytes and fibroblasts in co-culture. With decrease of cardiomyocytes ratio, heterocellular contacts remained unchanged. In average $n=136$ cells analized per cardiomyocytes/fibroblasts ratio. Images: Confocal fluorescence micrographs of co-cultures with four fold staining of $\alpha$-actinin in cardiomyocytes (red), vimentin in fibroblasts (green), DAPI for nuclei (blue) and Cx43 in electrical gap junctions (yellow or white). Top left: Magnified view of merged images of co-culture with $50 \%$ cardiomyocytes and the respective Cx43 (Top right). Scale bar $50 \mu \mathrm{m}$. Bottom left: Exemplary merged micrograph of cardiomyocytes monoculture and the homocellular junctions. Scale bar $50 \mu \mathrm{m}$. Bottom right: Exemplary merged magnified micrograph of co-culture with $20 \%$ cardiomyocytes. Scale bar $20 \mu \mathrm{m}$. 
figure 3.2 shows the results of the quantification and the spatial distribution of mechanical junction protein. On the top of the figure, the quantification of the N-Cad in the co-cultures with various ratios is given. The bottom part of the figure show four magnified confocal images, where the cardiomycytes are shown in red, the fibroblasts in green, the N-Cad proteins in yellow or white and the nuclei in blue. In $100 \%$ cardiomyocytes cultures $76 \pm 9 \%$ of the total amount of the visualised N-Cad was localized between cardiomyocytes, $23 \pm 10 \%$ coupling cardiomyocytes and fibroblasts and $1 \pm 1 \%$ between the residual fibroblasts. The $\mathrm{N}$-Cad staining at connecting cardiomyocytes is shown in the bottom-left micrograph in figure 3.2. In a co-culture with $20 \%$ fibroblasts the adhesion junctions are still prominently localized between cardiomyocytes (66 $\pm 5 \%$ ), whereas between cardiomyocytes and fibroblasts it was $33 \pm 5 \%$, and between fibroblasts it was $1.2 \pm 0.1 \%$. In co-cultures with 1:1 ratio, $52 \pm 17 \%$ of $\mathrm{N}$-Cad was observed in between cardiomyocytes, $33 \pm 4 \%$ between cardiomyocytes and fibroblasts, and 15 $\pm 2 \%$ between fibroblasts and fibroblasts. It is interesting to observe that fibroblasts can physically deform the membrane of cardiomyocytes through N-Cad junctions, as can be seen in top of the four micrographs of figure 3.2. In co-cultures with $20 \%$ cardiomyocytes, $73 \pm 7 \%, 21 \pm 4 \%$ and $6 \pm 2 \%$ of junctional $\mathrm{N}$-Cad was localized in regions between fibroblasts, cardiomyocytes and fibroblasts and cardiomyocytes, respectively. A visualization of the last configuration is shown in the bottom-right micrograph in figure 3.2. Similar to the case of the gap junctions, N-Cad was localized between all the three possible combinations of cell pairs. The amount of homocellular adhesion junctions raised together with the amount of the respective cell type. For example, in the case of co-culture with $20 \%$ cardiomyocytes the N-Cad localization between fibroblasts and fibroblasts was five times higher than in the case of $50 \%$ cardiomyocytes. Heterocellular adhesion junctions increased only marginally in a co-culture with $80 \%$ and $50 \%$ of cardiomyocytes.

In summary, in this work, both mechanical (N-Cad) and electrical (Cx43) junctions occurred between all the three possible combinations of cell interactions (cardiomyocytes cardiomyocytes, and fibroblasts-fibroblasts and cardiomyocytes-fibroblast) at any ratio of cardiomyocytes and fibroblasts. The occurrence of Cx43 and N-Cad between homocellular cell contacts varies proportional to the cardiomyocytes-fibroblasts ratio in the co-cultures, with however, one slight discrepancy: The amount of Cx43 junctions and $\mathrm{N}$-Cad junctions between fibroblasts-fibroblasts is respectively two and five folds higher than the co-culture with $50 \%$ cardiomyocytes, respectively. No significant difference in the fraction of the expressed N-Cad adhesion and Cx43-gap junctions was observed between fibroblasts in the co-culture with $20 \%$ cardiomyocytes. 


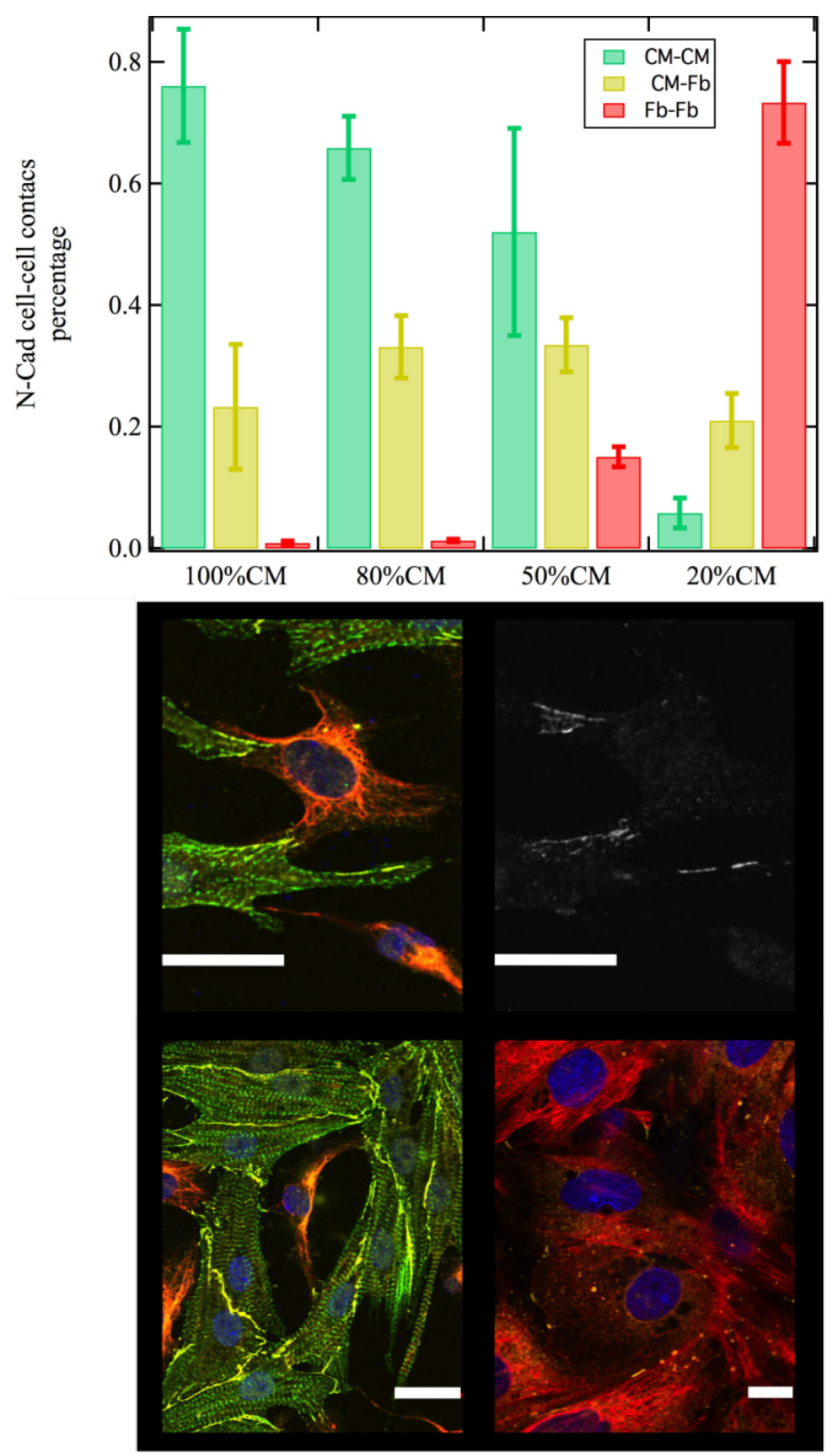

Figure 3.2: Mechanical junctions. Top graph: Distribution of heterocellular and homocellular mechanical coupling between cells in co-cultures with different cardiomyocyte(CM)-fibroblast (Fb) ratios. N-Cad, indicative of adhesion junctions was localized at the border between fibroblasts, cardiomyocytes and cardiomyocytes-fibroblasts. The amount of protein between homocellular electrical junctions cardiomyocytes-cardiomyocytes and fibroblasts-fibroblasts raised with respectively the increase of cardiomyocytes and fibroblasts in the co-culture. With decrease of cardiomyocytes ratio, heterocellular contacts slightly changed. In average $n=120$ cells were analysed per cardiomyocytes/fibroblasts ratio. Images: Confocal fluorescence micrographs of co-cultures with four fold staining of $\alpha$-actinin in cardiomyocytes (red), vimentin in fibroblasts (green), DAPI for nuclei (blue) and N-Cad in electrical gap junctions (yellow or white). Top left: Magnified view of merged images of co-culture with $50 \%$ cardiomyocytes and the respective N-Cad (Top right). Scale bar $20 \mu \mathrm{m}$. Bottom left: Exemplary merged micrograph of cardiomyocytes monoculture and the homocellular junctions. Scale bar $50 \mu \mathrm{m}$. Bottom right: Exemplary merged magnified micrograph of co-culture with $20 \%$ cardiomyocytes. Scale bar $50 \mu \mathrm{m}$. 


\subsubsection{Impedance Measurements}

The cardiomyocytes-fibroblasts connectivity was quantified by monitoring the barrier resistance of cellular monolayers. For this purpose different cardiomyocytes-fibroblasts ratios were seeded on a gold electrode. The impedance spectra obtained from the cocultures were fitted using a model that takes the rectangular shape of the cells into account as was introduced in section 2.3.1. Due to the capacitive nature of cellular membranes at low frequencies the impedance contribution in those frequencies arises from the cellular barrier resistance $R_{b}$ indicative of cardiomyocytes-cardiomyocytes / cardiomyocytes-fibroblasts / fibroblasts-fibroblasts connectivity. Mean values of the real part of the impedance at $4 \mathrm{kHz} Z_{\text {Renorm } @ 4 k H z}$ for cardiomyocytes-fibroblasts monolayers were analyzed by varying the ratio of fibroblasts in the co-cultures. The choice of this frequency value was due to the high signal-to-noise ratio for the covered electrode. Data were normalized for the value of impedance of the uncovered electrode. Figure 3.3A summarizes mean values of $Z_{\text {Renorm } @ 4 k H z}$ measured $120 \mathrm{~h}$ after seeding of the cells. The impedance increased with lower fractions of cardiomyocytes in co-cultures and it reached fibroblasts represented $90 \%$ of the cells. $R_{b}$ followed the same trend as $Z_{\text {Renorm } @ 4 \mathrm{kHz}}$ (figure 3.3B). After $120 \mathrm{~h}$ the highest value of $R_{b}$ was measured in co-culture with $90 \%$ fibroblasts $\left(R_{b}=12.6 \Omega \mathrm{cm}^{2}\right)$ representing the strongest connectivity in the co-culture. This value is two times higher than the connectivity of cardiomyocytes $\left(5.2 \Omega \mathrm{cm}^{2}\right)$ and fibroblasts $\left(5.7 \Omega \mathrm{cm}^{2}\right)$. (Impedance experiments performed by Dr. Marco Tarantola, Max Planck Institute for Dynamics and Self-Organization, Göttingen, Germany)
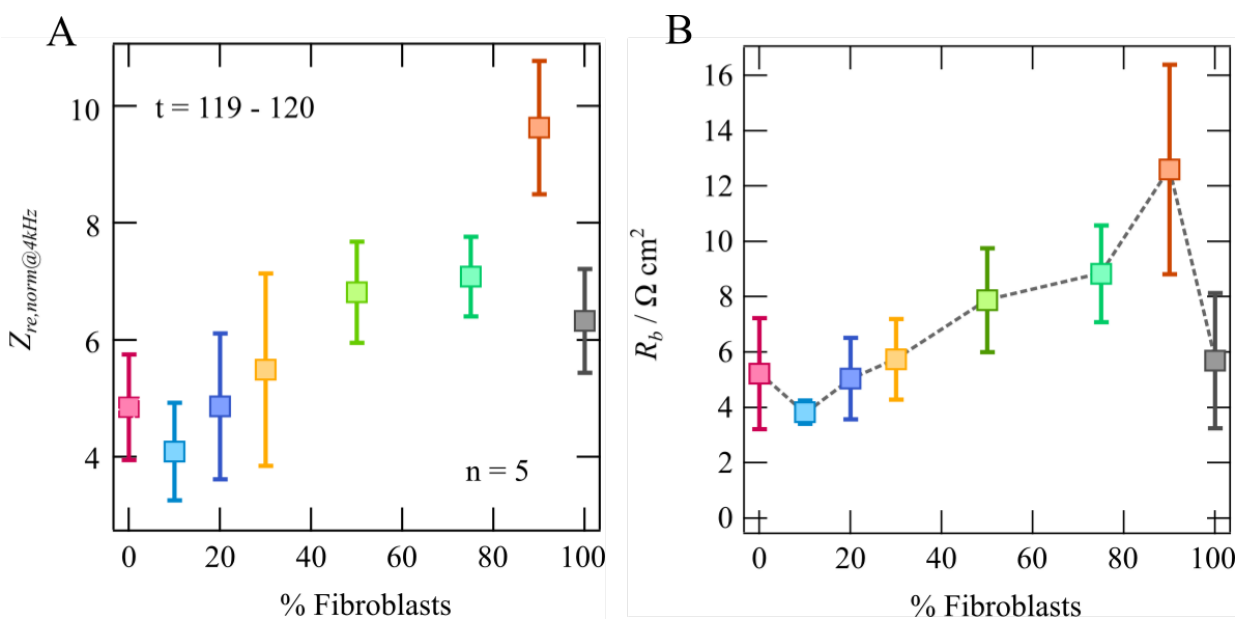

Figure 3.3: ECIS quantification of fibrotic connectivity. Mean $Z_{\text {Renorm } @ 4 k H z}$ values $(\mathbf{A})$ and mean values for cell-cell contact density parameter $R_{b}(\mathbf{B})$ for co-cultures with $0 \%, 10 \%, 20 \%, 30 \%, 50 \%$, $75 \%$, $90 \%$ fibroblasts five days after seeding. $n=5 \pm$ s.d.[43] Data analysis performed by Marco Tarantola, Max Planck Institute for Dynamics and Self-Organization, Göttingen, Germany, and Jan Rother, Institute of Physical Chemistry, Georg-August-University, Göttingen, Germany 


\subsubsection{Electromechanical Coupling}

To study the electromechanical coupling of cardiomyocytes-fibroblasts, in co-cultures with different ratios of fibroblasts, ECIS and PIPC were employed.

The spontaneous beating frequency was measured from the oscillation in $Z_{\text {Renorm }} 4 \mathrm{kHz}$ generated at each contraction due to the periodic fluctuations of the current underneath the cell basal membrane of the cell. Figure 3.4A shows an exemplary oscillation of the real part of the impedance measured from co-cultures with different ratios of fibroblasts. For $10 \%$ and $0 \%$ of cardiomyocytes no fluctuations in the impedance were recorded. High oscillation amplitudes were observed with high percentage of cardiomyocytes indicating a larger number of beating cells on the electrode. Using the values of $W$ and $L$ as mentioned in section 2.3.1 the number of cells covering each electrode with a diameter of $250 \mu \mathrm{m}$ was estimated to be around 32 .
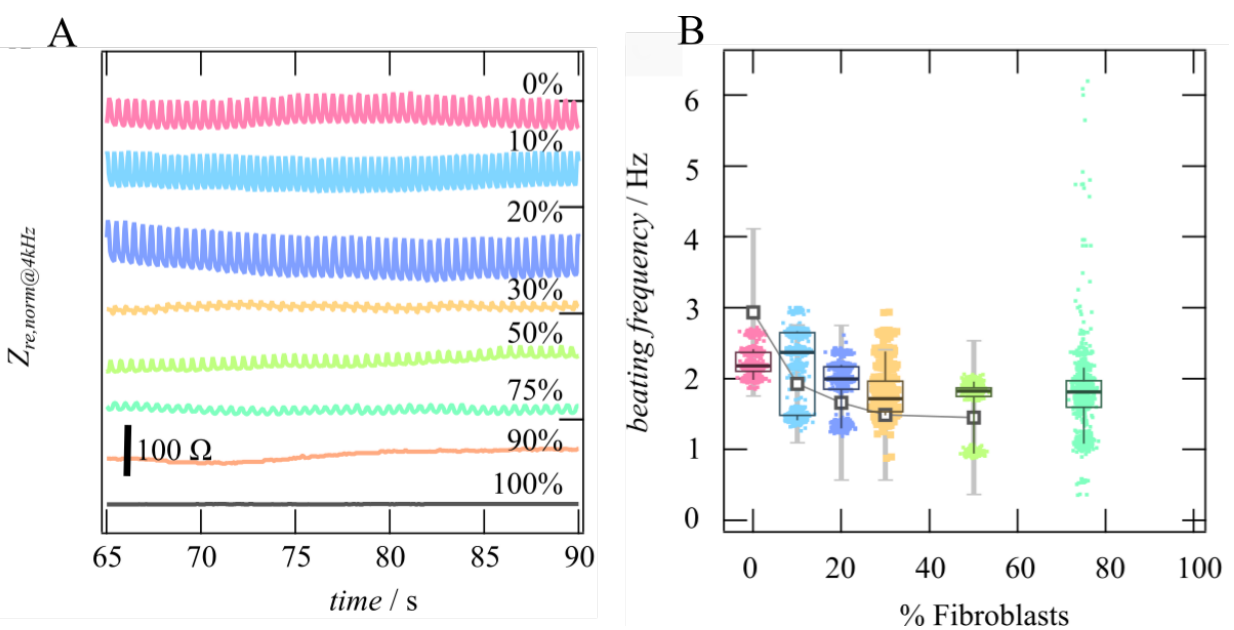

Figure 3.4: Beating coupling quantification via ECIS. A Exemplary time trace of the impedance for different fibroblasts fractions; $0 \%$ (pink), $10 \%$ (light blue), 20\% (purple), 30\% (yellow), 50\% (light green), $75 \%$ (dark green), $90 \%$ (orange), $100 \%$ (grey). B Beating frequency of co-cultures as function of fibroblasts ratio. Box plot with median value, $25 \%$ and $75 \%$ percentiles and $10 \%$ and $90 \%$ whiskers. $n=3$.[43] Data analysis performed by Marco Tarantola, Max Planck Institute for Dynamics and Self-Organization, Göttingen, Germany.

Figure $3.4 \mathrm{~B}$ presents the beating frequency as a function of fibroblasts ratio in a co-cultures. The observed decrease of co-culture beating frequency indicates that fibroblasts influences the electrophysiology of cardiomyocytes probably through both electrical and mechanical coupling. It is worth to mention here that like in a fibrotic tissue, in the co-culture used in this experiment fibroblasts had been activated and transformed into an contractile fibroblast phenotype called myofibroblasts. This was additionally confirmed by the expression of $\alpha$ smooth muscle actin ( $\alpha$-SMA) as shown in figure 3.5.

Fibroblasts functionally coupled with cardiomyocytes, inducing a decrease in beating frequency of the cellular monolayer. This phenomenon becomes more pronounced by increasing the ratio of the fibroblasts in co-culture. Contractile motion was observed 


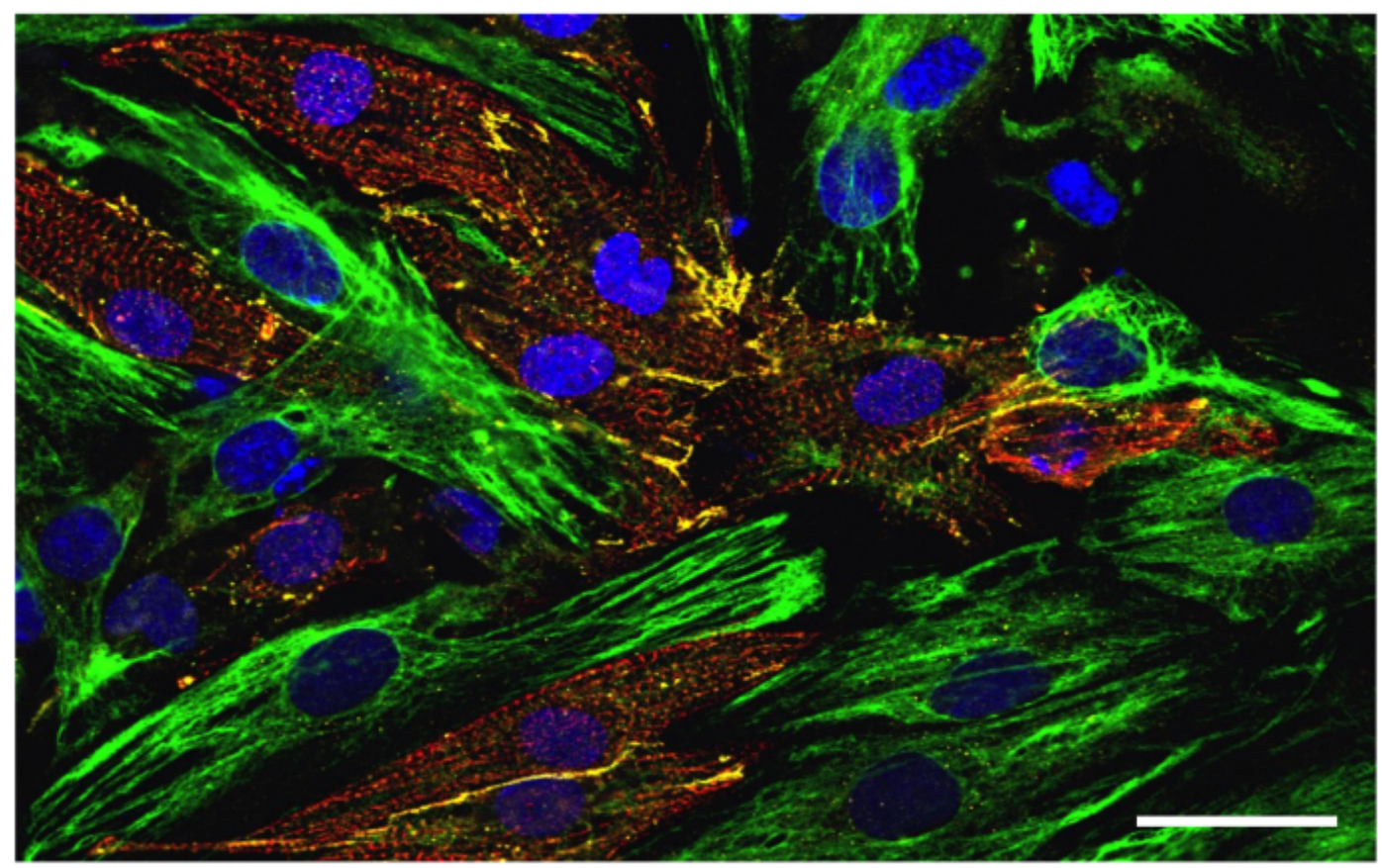

Figure 3.5: Myofibroblasts transformation. Exemplary confocal fluorescence of stained co-culture of cardiomyocites via $\alpha$-actinin (red), myofibroblasts via $\alpha$-SMA (green) and nuclei via DAPI (blue). Scale bar $30 \mu \mathrm{m}$.
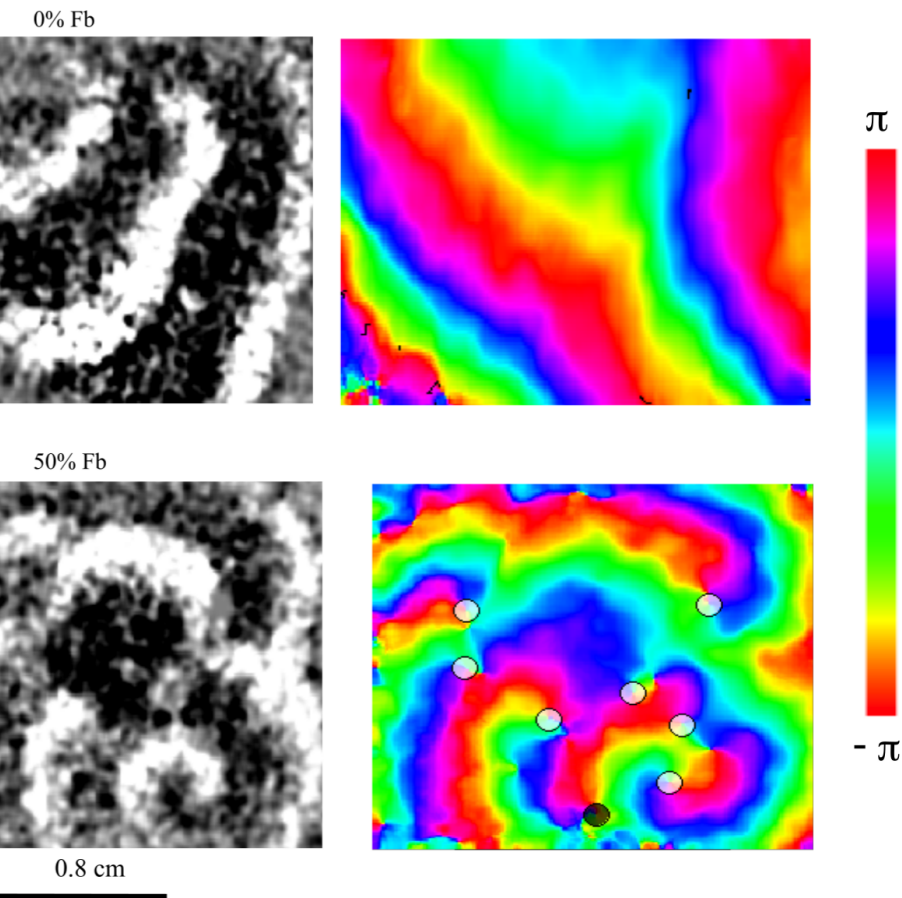

Figure 3.6: Functional coupling between cardiomyocytes and fibroblasts via phase contrast imaging. Exemplary processed images of cardiomyocytes monoculture and co-culture with $50 \%$ fibroblasts with the corresponding phase $\phi$ shifts from $-\pi$ to $+\pi . n=3$. [43] Image obtained by Dr. Claudia Richter, Max Planck Institute for Dynamics and Self-Organization, Göttingen, Germany. 
in mixtures up to $75 \%$ fibroblasts. Another evidence of the influence of fibroblasts on the electrophysiology of cardiomyocytes, can be inferred from phase-contrast imaging analysis of excitation wave propagation in cardiomyocyes-fibroblasts co-cultures. A gradual shift from quasi planar wavefront to spiral waves, due to the origin of phase singularities with increasing percentage of fibroblasts in the co-cultures was observed. Exemplary processed images for pure cardiomyocytes and mixture with $50 \%$ fibroblasts are shown in figure 3.6. (Impedance experiments and phase-contrast imaging were performed by Marco Tarantola and Claudia Richter, respectively, Max Planck Institute for Dynamics and Self-Organization, Göttingen, Germany)

\subsubsection{Microrheological properties of Cardiac Fibrosis Model}

The viscoelastic properties of primary neonatal rat cardiomyocytes, fibroblasts and coculture of cardiomyocytes and fibroblasts (1:1 ratio) were measured by using frequencydependent AFM-based microrheology. The results and an exemplary AFM deflection image of living cardiomyocytes and fibroblasts co-culture are shown in figure 3.7. The points in the image 3.7 indicate the positions where force-deformation curves were analyzed. Cells were kept five days in incubator before measurements. Storage $G^{\prime}$ and loss $G^{\prime \prime}$ moduli were determined from force-indentation curves by applying small oscillations at several frequencies $(5-150 \mathrm{~Hz})$ to the cantilever in contact with the cells, as explained in section 2.4.2.

Cardiomyocytes exhibited a $G^{\prime}$ of $1.7 \mathrm{kPa}$ at $5 \mathrm{~Hz} . G^{\prime}$ increased obeying a power-law relation over the frequency with an exponent of 0.35 , reaching a value of $6 \mathrm{kPa}$ at $150 \mathrm{~Hz}$. $G^{\prime \prime}$ for the same monocultures was two times lower than $G^{\prime}$ at $5 \mathrm{~Hz}$ with a value of $793 \mathrm{~Pa}$. It increased with frequency and reached a value of $7.5 \mathrm{kPa}$ at $150 \mathrm{~Hz}$, exceeding the value of $G^{\prime}$ (see figure 3.7A). Values of $G^{\prime}$ and $G^{\prime \prime}$ for cardiac fibroblasts were found to be lower than the ones for cardiomyocytes at all frequencies. $G^{\prime}$ was $693 \mathrm{~Pa}$ at $5 \mathrm{~Hz}$ and it increased exhibiting a weak power-law of 0.17 . The loss modulus $G^{\prime \prime}(233 \mathrm{~Pa}$ at $5 \mathrm{~Hz}$ ) was lower than $G^{\prime}$ for frequencies $<60 \mathrm{~Hz}$ and it reached a value twofold higher at $150 \mathrm{~Hz}$ $(2.93 \mathrm{kPa}$ ) (see figure 3.7B).Co-culture with $50 \%$ cardiomyocytes and $50 \%$ fibroblasts showed a frequency dependent increase of $G^{\prime}$ with a power-law exponent of 0.17 from $2.15 \mathrm{kPa}$ at $5 \mathrm{~Hz}$ to $4.25 \mathrm{kPa}$ at $150 \mathrm{~Hz}$ (see figure 3.7D). For cardiomyocytes, $\eta$ at lower frequencies was lower than 1 , indicating a solid-like behavior of the cells. With increasing frequencies, $\eta$ became larger until it reached values higher than 1 starting from $80 \mathrm{~Hz}$, at which cells express a more fluid-like nature. A similar trend was observed for $G^{\prime \prime}$ with the exception that the co-culture $G^{\prime \prime}$ exceeded the values of the cardiomyocytes monoculture only below $20 \mathrm{~Hz}$.

The figure 3.8 shows the loss tangent $\eta$ which represents the ratio between $G^{\prime \prime}$ and $G^{\prime}$ for monocultures and co-culture. In the case of the cardiomyocytes co-culture, $\eta$ values 

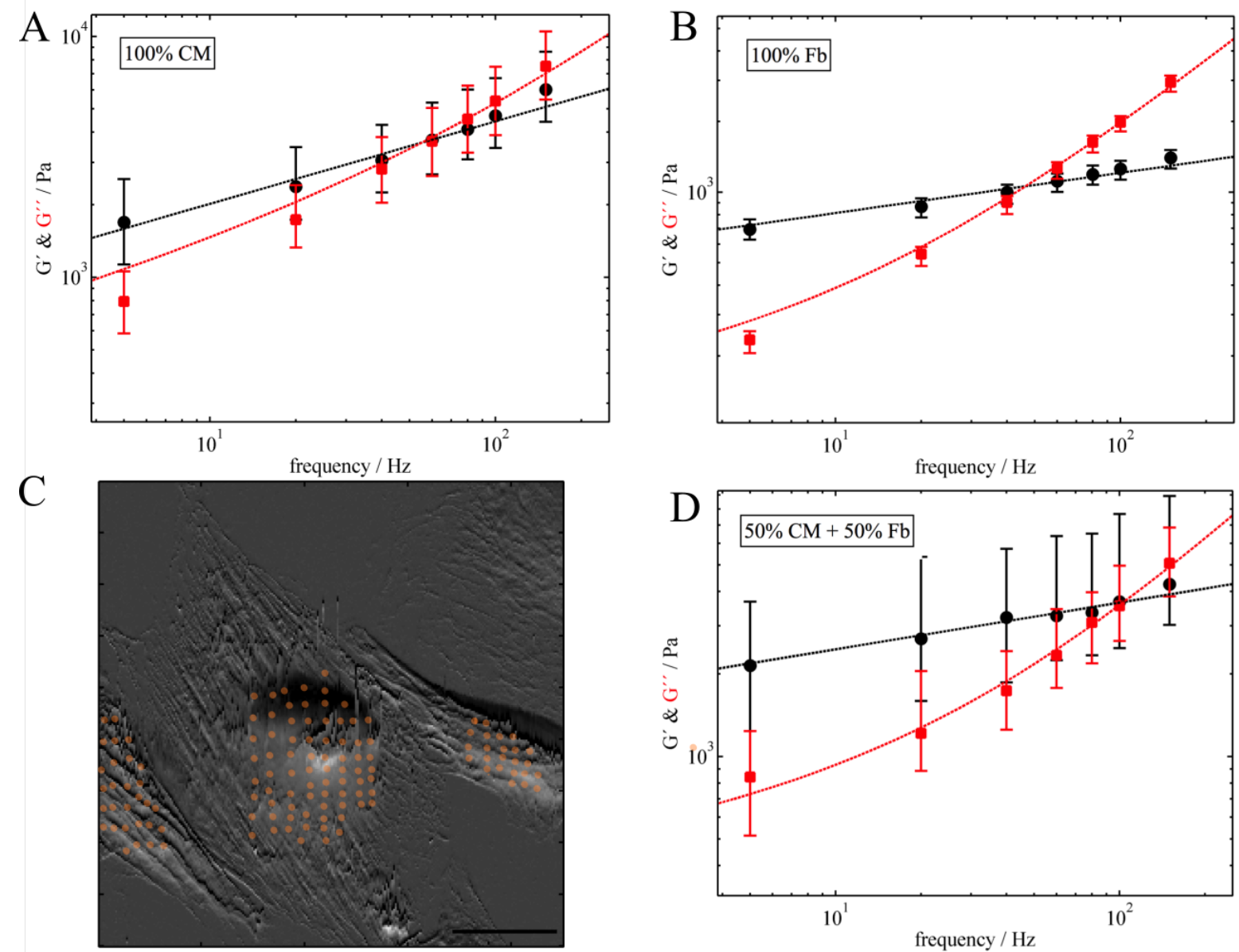

Figure 3.7: Mechanics of cardiomyocytes and fibroblasts monocultures and co-cultures. Frequency dependency of storage modulus $G^{\prime}$ (black) and loss modulus $G^{\prime \prime}$ (red) for cardiomyocytes $(\mathrm{CM})(n=5)$ $(\mathbf{A})$, fibroblasts $(\mathrm{Fb})(n=3)(\mathbf{B})$ and cardiomyocytes-fibroblasts co-culture $(n=3)(\mathbf{D})$. Solid lines show the power-law structural damping model fit. Data are shown as median \pm quartiles. (C)Exemplary AFM deflection image of living cardiomyocyte between two fibroblasts. Points mark the positions where the force- indentation curves were analysed. Scale bar $20 \mu \mathrm{m}$

was below 1 until $80 \mathrm{~Hz}$ indicating a solid-like behavior, whereas at higher frequency $\eta$ increased and cells expressed a more fluid-like nature. On the other hand, for fibroblasts monocultures the solid-liquid-like transition frequency occurs at $60 \mathrm{~Hz}$. As can be seen from the trend shown in figure 3.8 the $\eta$ values of cardiomyocytes is higher than the values for fibroblasts at frequencies lower than $40 \mathrm{~Hz}$. The co-culture showed values of $\eta$ lower than both the monocultures at all frequencies and remained less than 1 up to $150 \mathrm{~Hz}$, indicating a solid-like behavior throughout the frequency range.

Rheological data were fitted with the power-law structural damping model (equation 2.20) with three independent fitting parameters: $G_{0}$, overall stiffness of the cells, the cellular viscosity $\mu$ and $\beta$ representing the power-law coefficient. The fitted values for the three cell cultures are shown in table 3.1. $\beta$ is related to the agitation of cytoskeleton elements. $G_{0}$ exhibits the same trend as $G^{\prime}$. The stiffness of cardiomyocytes monocultures $G_{0}=601 \mathrm{~Pa}$ appeared to be 1.5 time larger than the fibroblasts monocul- 


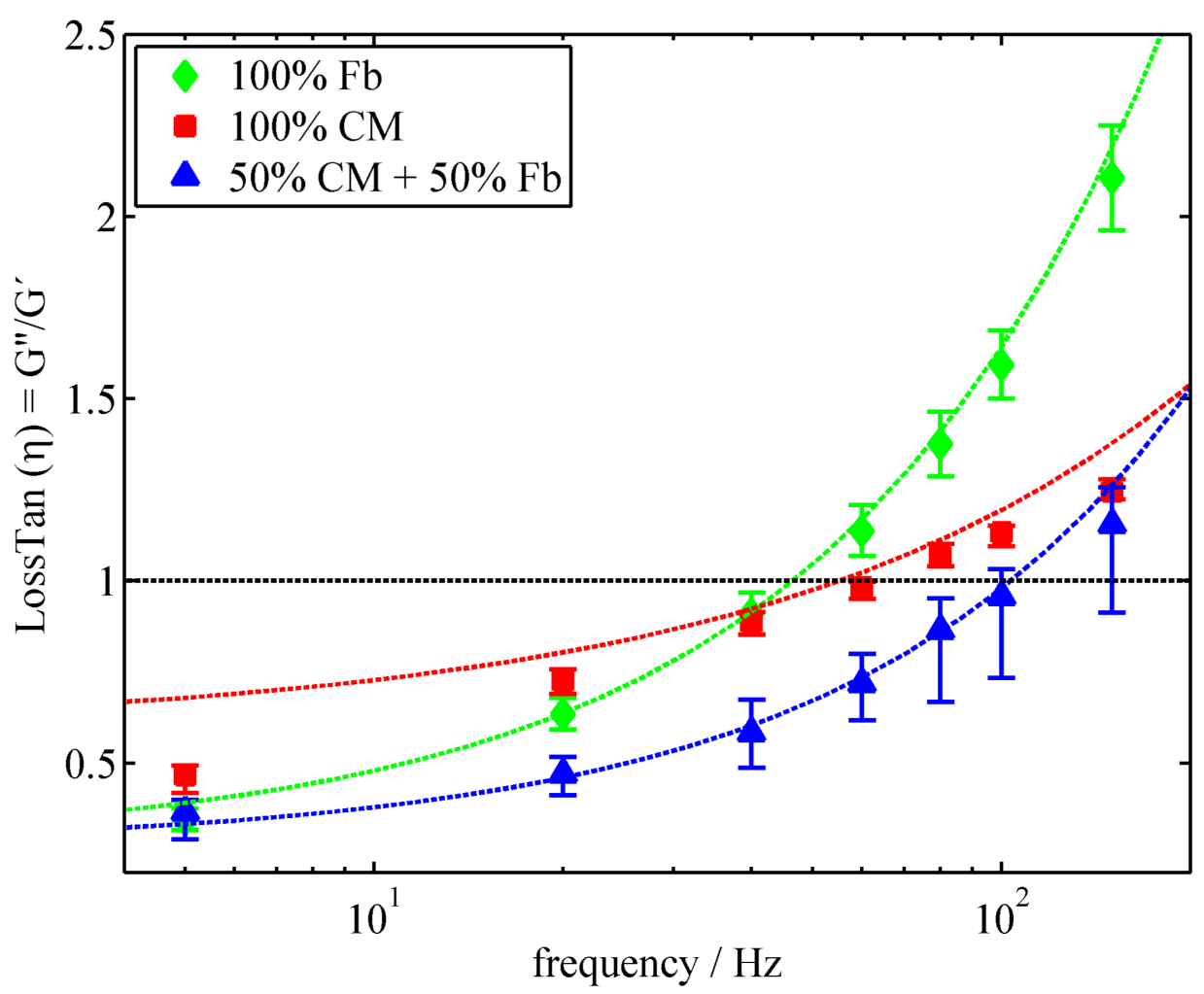

Figure 3.8: Frequency dependency of loss tangent for cardiomyocytes $(\mathrm{CM})(n=5)$ (red), fibroblasts $(\mathrm{Fb})(n=3)$ (green) and cardiomyocytes-fibroblasts co-culture $(n=3)$ (blue). Data are shown as median \pm quartiles.

ture and it increased additionally in a $1: 1$ ratio co-culture $\left(G_{0}=1.2 \mathrm{kPa}\right)$. The viscosity of the co-culture was similar to the cardiomyocytes monoculture and $\beta$ similar to the value of fibroblasts monoculture $(\beta=0.17)$.

\begin{tabular}{cccc}
\hline & $G_{0} \pm \mathbf{S E} / \mathbf{P a}$ & $\beta \pm \mathbf{S E}$ & $\mu \pm \mathbf{S E} / \mathbf{P a} \mathbf{~ s}$ \\
\hline $100 \%$ cardiomyocytes & $601 \pm 182$ & $0.35 \pm 0.05$ & $4.9 \pm 1.2$ \\
100\%fibroblasts & $399 \pm 41$ & $0.17 \pm 0.02$ & $2.6 \pm 0.09$ \\
$50 \%$ cardiomyocytes-50\%fibroblasts & $2150 \pm 110$ & $0.17 \pm 0.01$ & $4 \pm 0.2$ \\
\hline
\end{tabular}

Table 3.1: Fitting parameters of the power-law structural damping model of the rheological data of monocultures and co-culture of cardiomyocytes and fibroblasts. $G_{0}$ describes the overall stiffness of the cells, $\alpha$ is the power-law coefficient and $\mu$ the cellular viscosity.

\subsection{Effect of shear stress on cardiac laminar sheets}

The results presented in this subsection show the effect of high shear stresses on cardiomyocytes monocultures and cardiomyocites/fibroblasts co-cultures, as it occurs in the early stage of ventricular remodeling induced by laminar fluid flow. The values of shear stress were chosen an order of magnitude higher than the physiological value estimated in vivo to mimic pathological conditions as explained in subchapter 2.2.1. The cellular 
response was analysed during two defined time windows to probe the immediate-short term and long term effect of the shear stress. For the short term effect a $3 \mathrm{~h}$ window (30 min before and $2.5 \mathrm{~h}$ after the flow onset) was analysed whereas for the long term effect a time window up to $108 \mathrm{~h}$ of shear stimulation was chosen (for details see section 2.3.3). For both time windows, cellular morphological changes and collective contractile behaviour, induced by high shear stresses were studied employing ECIS, RICM and CLSM.

\subsubsection{Immediate-short term effect of shear stress on cellular morphology}

\section{Impedance measurements}

Impedance of the cardiomyocytes monolayers seeded on the microelectrodes subjected to laminar flow was measured as described in section 2.3.2. An example of the time course of the normalized real part of the impedance at $4 \mathrm{kHz}$ measured on the monolayer exposed to $1.59 \mathrm{dyn} / \mathrm{cm}^{2}$ shear stress is shown in figure 3.9. In the same image, the exponential-linear fit and the $Z_{\text {Renorm } @ 4 k z}$ of non-stimulated cardiomyocytes are presented in blue and black, respectively. A sharp increase in cardiomyocytes monolayer electrical impedance was observed concurrently with the shear stress application. On the contrary, the impedance of non-stimulated cells remained unchanged over the same

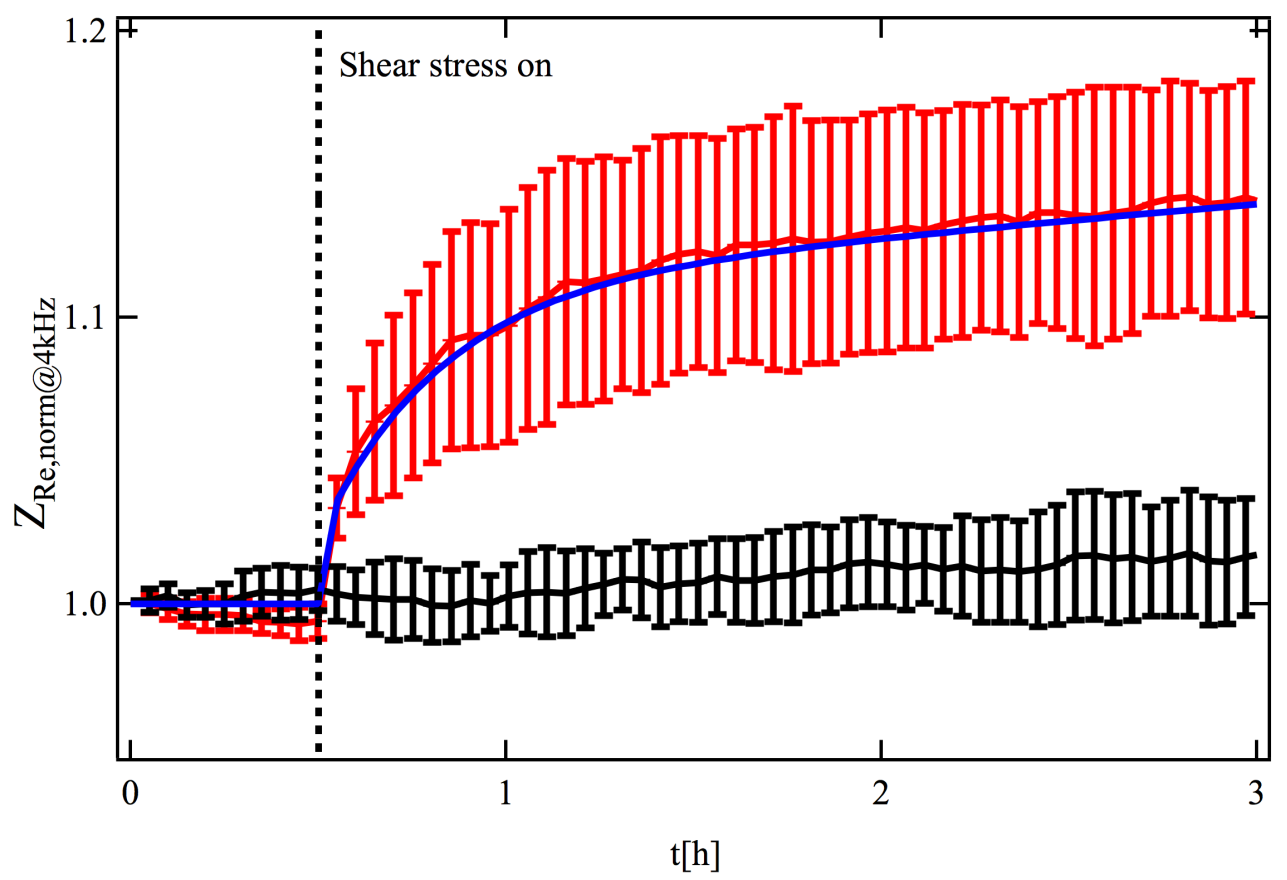

Figure 3.9: Impedance at the flow onset. Mean time course of the real part of the impedance recorded at $4 \mathrm{kHz}$ for cardiomyocyte monolayer with (red) and without (black) fluid flow stimulation and the fitted curve (blue) of the response to shear stress. The error bars represent the s.d. of $n=8$ electrodes (250 cells). The applied shear stress was $1.59 \mathrm{dyn} / \mathrm{cm}^{2}$ and data are shown in a $3 \mathrm{~h}$ time window, $30 \mathrm{~min}$ before and $2.5 \mathrm{~h}$ after the flow onset. 
time interval. In order to quantify the dynamics of the cellular response, the impedance trace (mean of $\mathrm{n}=8$ electrodes) at $4 \mathrm{kHz}$ was modelled with a combination of exponential and linear rise. The initial exponential rise is characterised by the rise time constant $\tau$ defined as the time to reach $63 \%$ of the maximum value (system's speed of response).

The influence of increased shear stress on cardiomycytes monolayer was investigated by applying values of $1.59 \mathrm{dyn} / \mathrm{cm}^{2}, 4 \mathrm{dyn} / \mathrm{cm}^{2}$ and $6 \mathrm{dyn} / \mathrm{cm}^{2}$. The short-term cellular response dynamics accelerated when the shear stresses was increased. The rise time constants for the shear stresses applied are listed in table 3.2. The $\tau$, for the shear of $4 \mathrm{dyn} / \mathrm{cm}^{2}$ is 5 folds higher than the value when $1.59 \mathrm{dyn} / \mathrm{cm}^{2}$ shear was applied. An increase of 2.5 folds in the shear stress $\left(4 \mathrm{dyn} / \mathrm{cm}^{2}\right)$ triggers a faster cell response, whereas no significant difference was observed in system's speed of response between $4 \mathrm{dyn} / \mathrm{cm}^{2}$ and $6 \mathrm{dyn} / \mathrm{cm}^{2}$.

To verify whether the movement of ions present in the cell medium influences the impedance when a shear stress is applied, two control experiments were performed by applying shear stress of $4 \mathrm{dyn} / \mathrm{cm}^{2}$ to i) uncoated ECIS Flow Array and ii) ECIS Flow Array coated with Gelatin-fibronectin only. The results, presented in figure 3.10 show

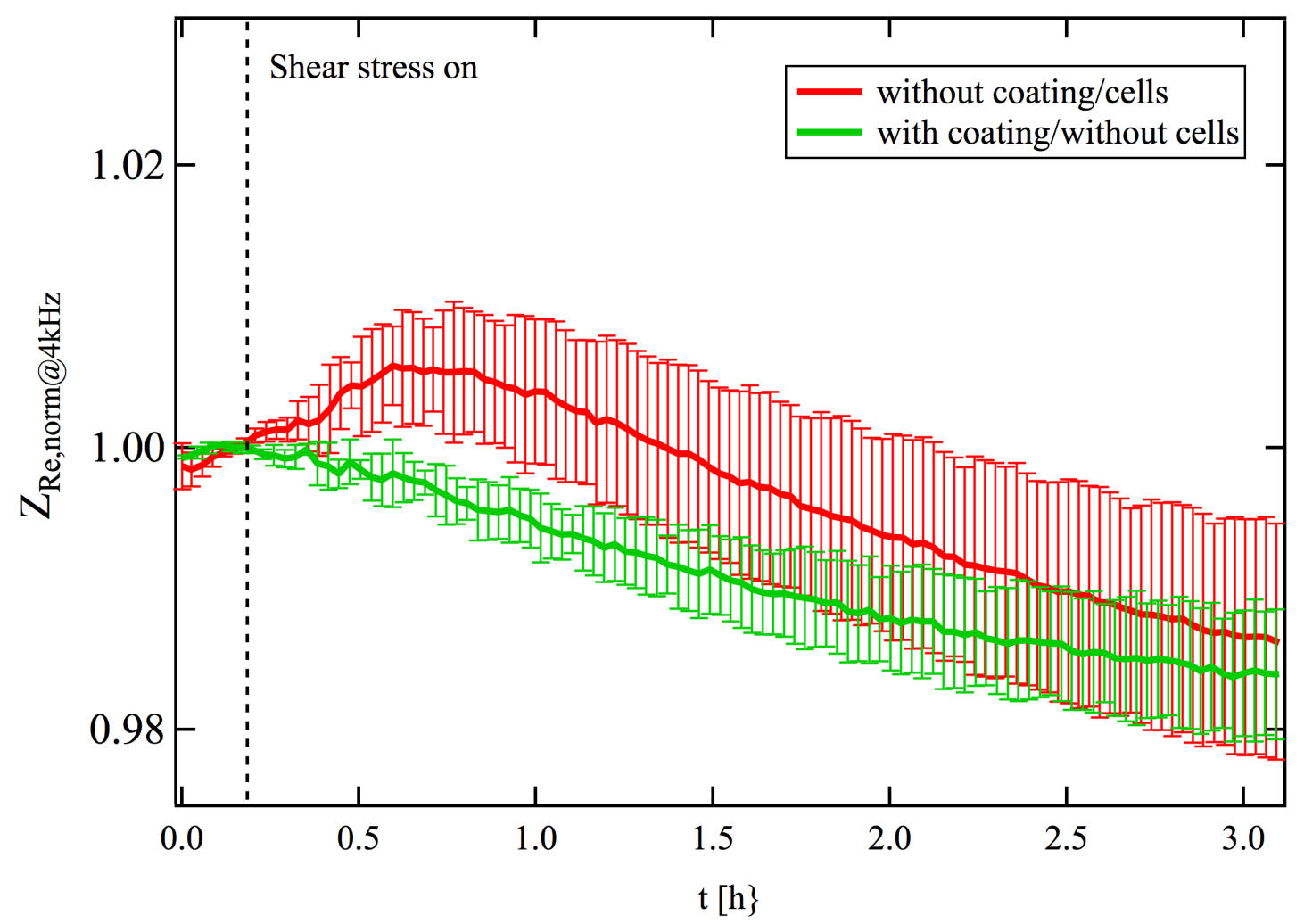

Figure 3.10: Impedance of uncoated and coated samples under shear stress. Mean time course of the real part of the impedance at $4 \mathrm{kHz}$ for uncoated (red) and Gelatin-fibronectin coated (green) electrodes when a shear stress of $4 \mathrm{dyn} / \mathrm{cm}^{2}$ was applied. $n=8 \pm$ s.d. 


\begin{tabular}{cccc}
\hline Parameter & $\mathbf{1 . 5 9}$ dyn $/ \mathrm{cm}^{2}$ & $\mathbf{4 d y n} / \mathrm{cm}^{2}$ & $\mathbf{6}$ dyn $/ \mathrm{cm}^{2}$ \\
\hline$\tau$ & $35 \pm 5 \mathrm{~min}$ & $7 \mathrm{~min} \pm 1$ & $6.6 \pm 0.6 \mathrm{~min}$ \\
\hline
\end{tabular}

Table 3.2: Parameters obtained from the linear-exponential fit (chapter 2.3.3) describing the dynamic response of $Z_{\text {Renorm } @ 4 k H z}$ to increasing value of shear stress. $\tau$ is a measure of speed of response.

a decrease in the electrical impedance during exposure to the laminar flow in both the cases, confirming that the complex impedance increase upon shear stress in the cell seeded ECIS Flow Array is indeed due to the active presence of the cell monolayer.

\section{RICM measurements}

RICM images were recorded in order to quantify cell-substrate distance and cell-contact area changes as an immediate response to the applied shear stress. As explained in section 2.4.3.2, images were analysed every $15 \mathrm{~min}$ for $3 \mathrm{~h}$ to extract the intensity histogram of the cell, to quantify cell contact area and qualitatively measure the cell-substrate distance. Each intensity trace was normalized to its mean intensity values. The figure 3.11 presents the mean of the normalized intensity traces for cells sheared at $4 \mathrm{dyn} / \mathrm{cm}^{2}$. The mean cell intensity decreases slightly when the shear stress is applied, reaching a minimum of $5 \%$ below the initial value (30 min before the flow onset). Afterwards, it increases again reaching the initial intensity in approximately $1.5 \mathrm{~h}$. The results suggest

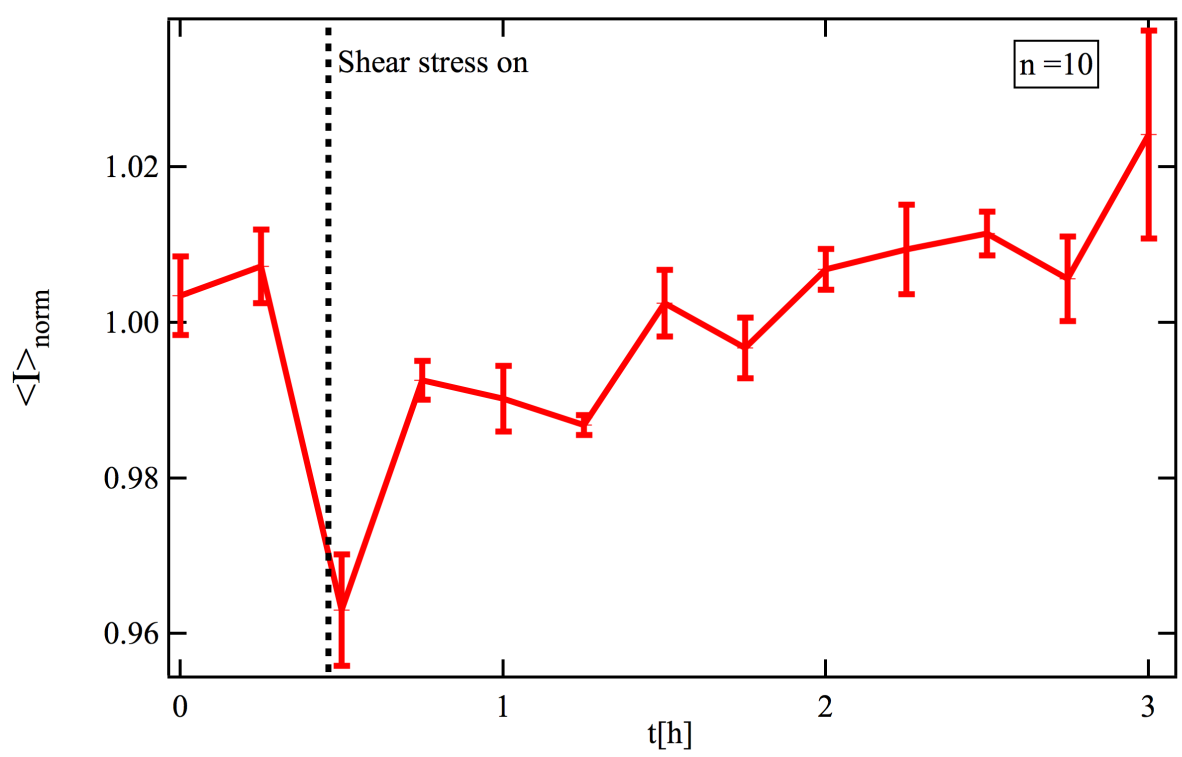

Figure 3.11: RICM intensity at the flow onset. Mean RICM intensity of sheared cells $\left(4 d y n / \mathrm{cm}^{2}\right)$ as function of time. The intensity of each trace was normalised by the mean of the trace. The figure shows the mean for $n=10 \pm$ s.d. A sharp decrease in normalised mean intensity follows the exposure of cardiomyocytes to flow. 
that when a cell monolayer is exposed to shear stress, suddenly the cells approach the glass surface and the distance between the ventral part of the cell and the surface is reduced. This means that a larger interference between glass/medium and medium/cell membrane reflected light occurs resulting to a decreased of the intensity.

In order to investigate if the immediate exposure to the flow induces only a decrease in cell-substrate distance, or if it also promotes cell spreading, the size of the projected cellular area was measured in the same time window. The results are shown in figure 3.12 when the shear stress was applied: no significant change in cell- substrate contact area occurs for $2.5 \mathrm{~h}$, and by the end of $3 \mathrm{~h}$ it increases to 1.2 times the original value reached within the first $30 \mathrm{~min}$. These results indicate that when the monolayer is suddenly sheared, the cells are pushed against the substrate without any immediate changes in contact area, whereas after approximately $2.5 \mathrm{~h}$ the cardiomyocytes start slightly to further spread.

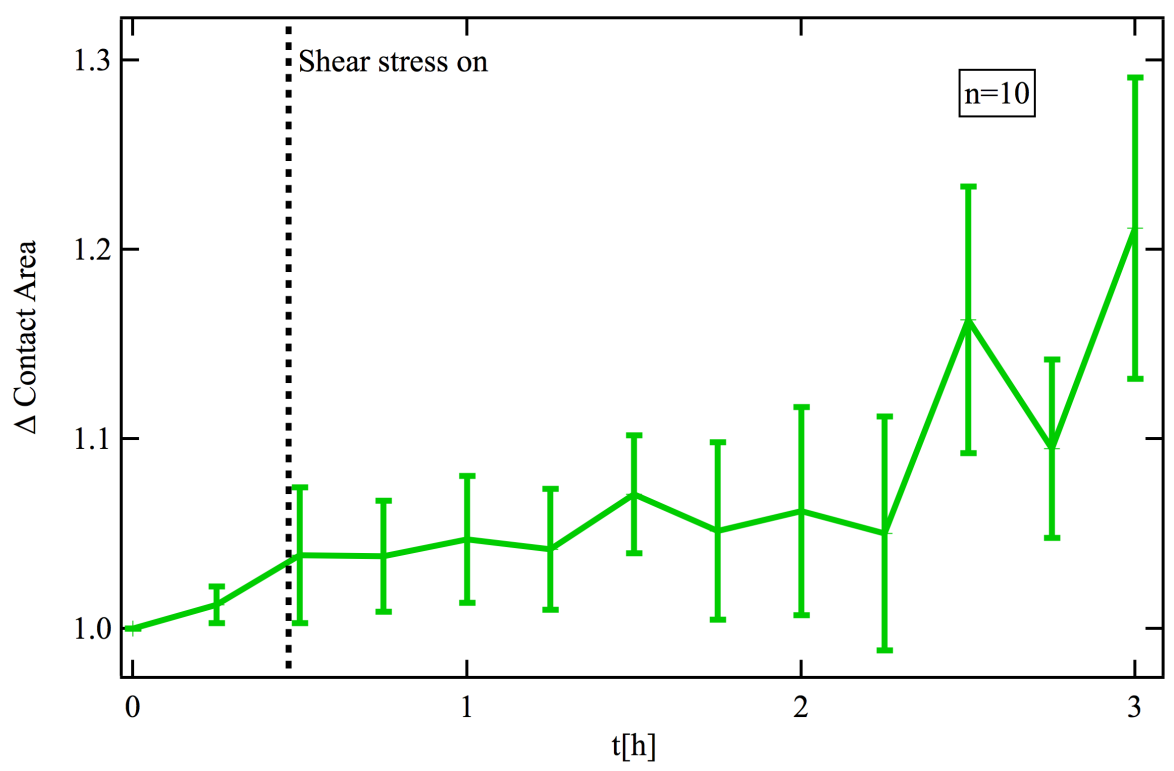

Figure 3.12: Cell-substrate contact area under short- term shear stress exposure. Time course of $4 d y n / \mathrm{cm}^{2}$-sheared cell-substrate contact area increment. Each cell area was normalised for its initial value in frame recorded $30 \mathrm{~min}$ before the cardiomycytes were exposed to flow. No significant changes in the cell-substrate contact area occur within $2 \mathrm{~h}$ from the shear onset for $\mathrm{n}=10 \pm$ s.d.

\subsubsection{Immediate-short term effect of shear stress on cellular contractility}

In order to asses whether spontaneous beating frequency of cardiomyocytes monolayer was immediately affected by the application of laminar flow, the real part of the impedance, measured in RTC-mode, was analysed as reported in section 2.3.3. The figure 3.13 presents on the left, the oscillatory micromotions visible in the $Z_{\text {Renorm } @ 4 k H z}$ time course at the flow onset $\left(\mathrm{t}=20 \mathrm{~s}\right.$ ) of $1.59 \mathrm{dyn} / \mathrm{cm}^{2}$, and on the right, the beating frequency of the cells before and after the application of different shear stresses. The presence of shear 

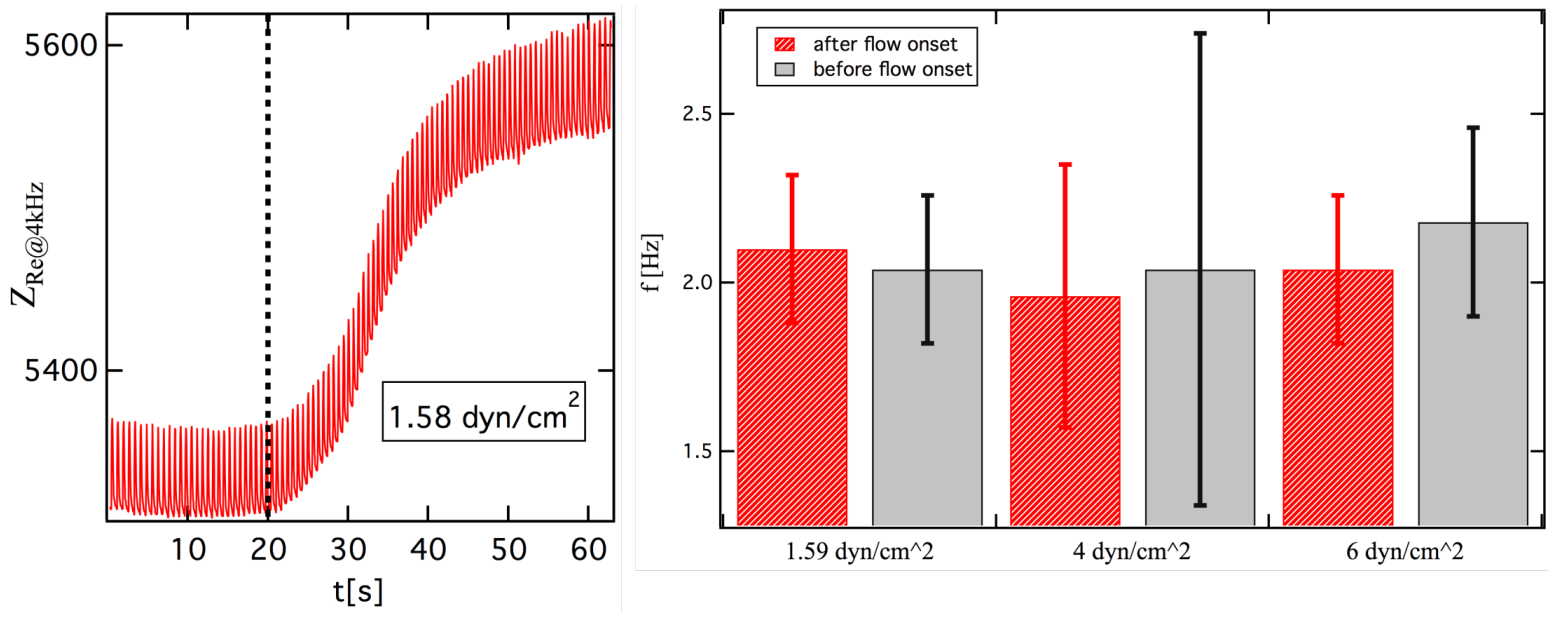

Figure 3.13: Cardiomyocytes beating frequency at the flow onset. Right: Cardiomycytes beating frequency as function of shear stress, before and after flow exposure. For every applied shear stress values, no changes in cellular beating rate after the flow onset are observed. Left: Exemplary oscillatory impedance time series measured on a cell-covered electrode with cardiomyocytes monolayer exposed to shear stress of $1.59 \mathrm{dyn} / \mathrm{cm}^{2}$. Dashed line indicates the flow onset. Mean and width of the peak from FFTs are plotted.

stress does not seem to affect the beating induced impedance fluctuations, whereas value of impedance increases in accordance with the results presented in the previous section. Regardless of the applied value of shear stress, cells beat with the same frequency of about $2 \mathrm{~Hz}$ both before and after the flow onset, suggesting that the sudden exposure to flow does not perturb the cardiomyocytes functionality.

\subsubsection{Long-term effect of shear stress on cells morphology}

\section{Impedance measurements}

A continuous increase in the real part of the impedance of the cardiomyocytes monolayers at $4 \mathrm{kHz}$ under fluid flow conditions was observed during the whole period of stimulation $(108 \mathrm{~h})$, as shown in figure 3.14. The increment of the mean $Z_{\text {Renorm } @ k H z}$ of cells sheared at $1.59 \mathrm{dyn} / \mathrm{cm}^{2}$ was 2.2 times higher than the value before the flow onset. When shear stress of $4 \mathrm{dyn} / \mathrm{cm}^{2}$ and $6 \mathrm{dyn} / \mathrm{cm}^{2}$ were applied, the final increase in the mean resistive part of the impedance was 3.03 and 3.06, respectively.

These results suggest that the cardiomyocytes monolayers under a long-term exposure to flow stimulation undergo morphological changes which affect, consequently, the current path underneath, around, in between, and through the cells resulting in higher values of impedance. Increasing the shear stress from $1.59 \mathrm{dyn} / \mathrm{cm}^{2}$ to $4 \mathrm{dyn} / \mathrm{cm}^{2}$ induces more pronounced changes in the impedance and therefore in cell morphodynamics, whereas the effect of $4 \mathrm{dyn} / \mathrm{cm}^{2}$ and $6 \mathrm{dyn} / \mathrm{cm}^{2}$ shear stress seems to be comparable. These findings are consistent with the results of the short-term effect of different shear stress values on the cellular dynamics response to the flow onset. 


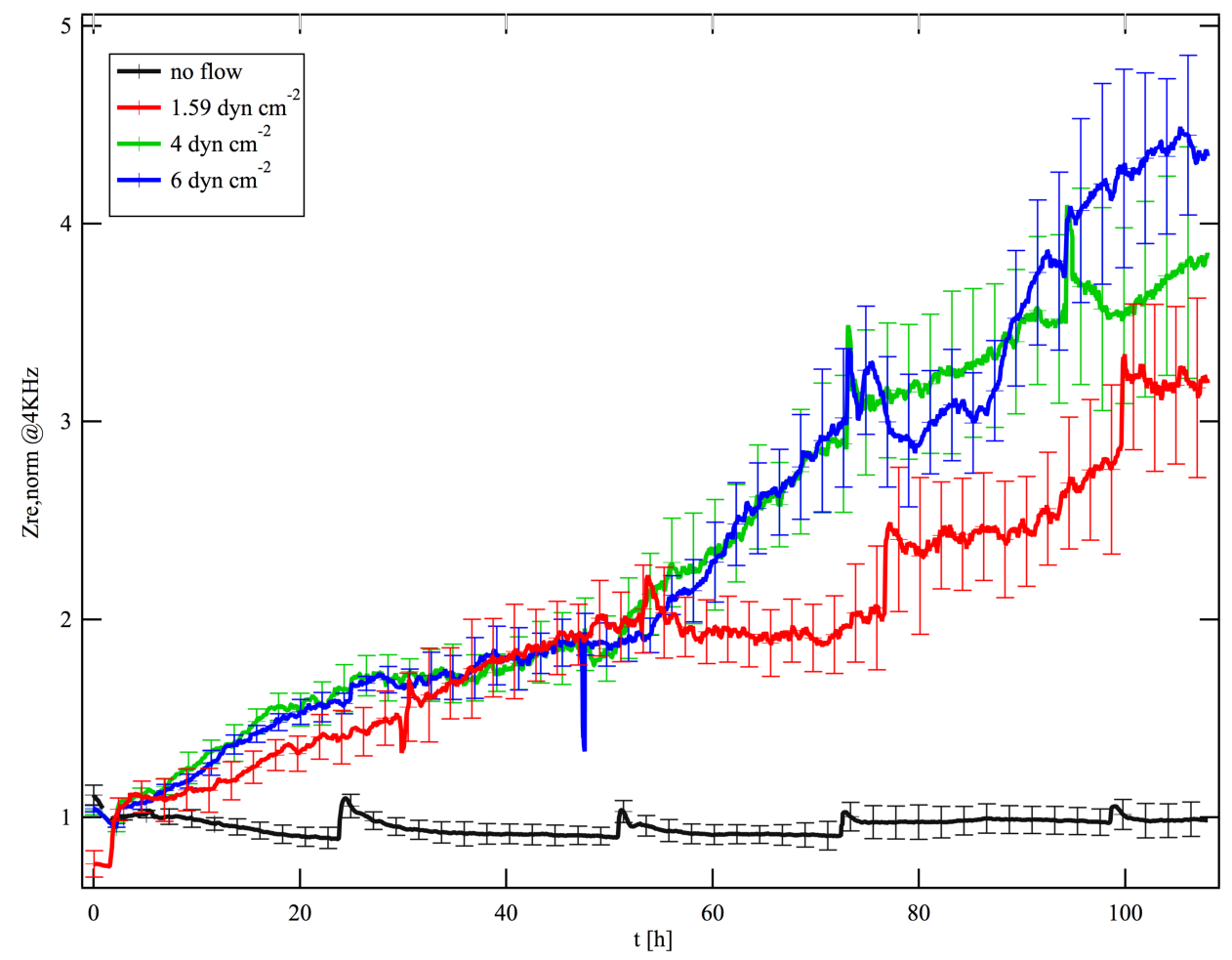

Figure 3.14: Impedance of cardiomyocytes under long-term exposure to different shear stresses. Mean time course of the real part of the cardiomyocytes monolayer impedance sheared at $1.59 \mathrm{dyn} / \mathrm{cm}^{2}$ (red) ( $n=7$ electrodes), $4 \mathrm{dyn} / \mathrm{cm}^{2}$ (green) ( $n=7$ electrodes), $6 \mathrm{dyn} / \mathrm{cm}^{2}$ (blue) ( $n=8$ electrodes) and without fluid stimulation (black) ( $n=8$ electrodes). Data are show as mean \pm s.d.

The measured data were fitted with the equation 2.7 to correlate the changes in the resistive part of the monolayer complex impedance with morphological parameters such as cell shape, cell-cell and cell-substrate junctions.

Figure 3.15 shows the values of the model parameters, $R_{b}, C_{m}$ and $\alpha$, at $48 \mathrm{~h}$ and $108 \mathrm{~h}$ after the flow stimulation onset for sheared $\left(1.59 \mathrm{dyn} / \mathrm{cm}^{2}\right)$ and not-sheared cardiomyocytes monolayers (right). The left side of the image shows how the parameters change with increasing the applied shear stress.

The junctional barrier resistance $R_{b}$ of sheared cardiomyocytes at $1.59 \mathrm{dyn} / \mathrm{cm}^{2}$ increased by a factor of 1.5 compared to the control cultures both at $48 \mathrm{~h}$ and $108 \mathrm{~h}$ after the flow stimulation started (figure 3.15a). On the other hand at $\mathrm{t}=48 \mathrm{~h}$ the cell membrane capacitance $C_{m}$ was 3.7 times lower when shear stress was applied than the control $\left(3 \pm 0.3 \mu \mathrm{F} \mathrm{cm} \mathrm{cm}^{-2}\right)$, and it decreased over the time reaching a value of $0.7 \pm$ $0.1 \mu \mathrm{F} \mathrm{cm}-2$ at $\mathrm{t}=108 \mathrm{~h}(3.15 \mathrm{c}) . \alpha$, related to the average height between the basal cell membrane and the substrate, was approximately $0.7 \pm 0.1 \Omega^{-1 / 2} \mathrm{~cm}$ for not sheared cardiomyocytes, both at $48 \mathrm{~h}$ and $108 \mathrm{~h}$, and under fluid flow stimulation increased 3.4 times (figure 3.15e). The average cell-substrate distance was estimated to be $\sim 150 \mathrm{~nm}$ in control conditions and $\sim 110 \mathrm{~nm}$ under shear flow conditions. Higher values of shear stress $\left(4 \mathrm{dyn} / \mathrm{cm}^{2}\right.$ and $\left.6 \mathrm{dyn} / \mathrm{cm}^{2}\right)$ did not induce significant changes in the model para- 

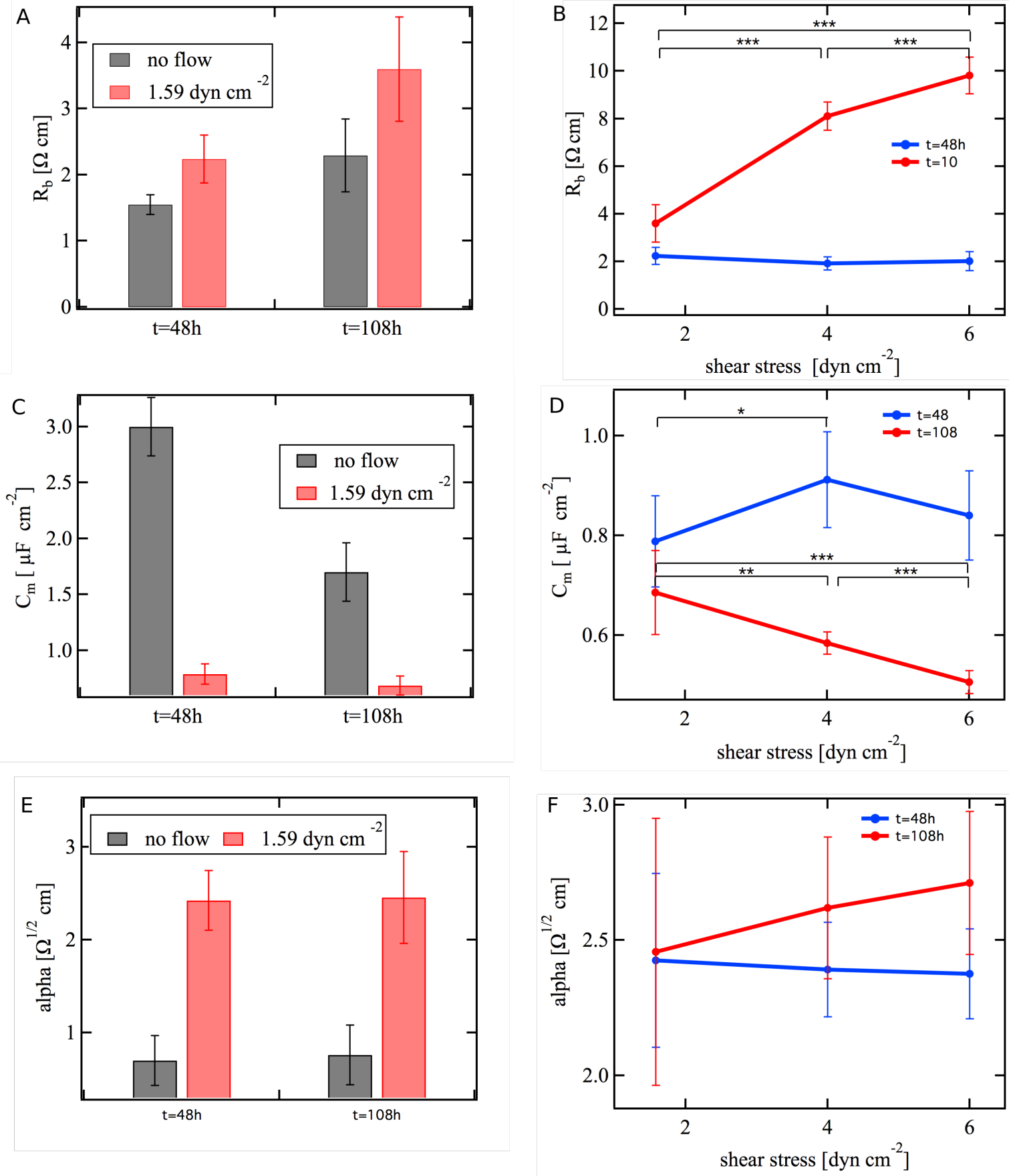

Figure 3.15: ECIS model parameters for cardiomyocytes non-exposed and exposed to different shear stresses. $R_{b}, C_{m}$ and $\alpha$ for sheared (red) and not sheared (black) cardiomyocyte monolayers after $48 \mathrm{~h}$ and $108 \mathrm{~h}$ of flow stimulation $(\mathbf{A}),(\mathbf{C}),(\mathbf{E})$. No flow $n=3$ electrodes, flow $n=7$ electrodes. $R_{b}, C_{m}$ and $\alpha$ as a function of shear stress values after $48 \mathrm{~h}$ (blue) and $108 \mathrm{~h}$ (red) of flow stimulation(B), (D), (F). For $1.59-4 \mathrm{dyn} / \mathrm{cm}^{2} n=7$ electrodes, $6 \mathrm{dyn} / \mathrm{cm}^{2} n=8$ electrodes. Data are shown as mean $\pm \mathrm{sd}$.

meters after $48 \mathrm{~h}$ of flow stimulation (blue curve in the graphs on the right column of 3.15). Conversely, after $108 \mathrm{~h}$ of exposure to the flow on the cardiomyocytes, increasing the shear stress from $1.59 \mathrm{dyn} / \mathrm{cm}^{2}$ to $4 \mathrm{dyn} / \mathrm{cm}^{2}$ (or $6 \mathrm{dyn} / \mathrm{cm}^{2}$ ), promoted 2.4 and 2.7 times higher values of cell-cell connectivity, $R_{b}(P<0.001)$, respectively. At $108 \mathrm{~h} C_{m}$ 
linearly decreased with high shear stress values (by $30 \%$ for $6 \mathrm{dyn} / \mathrm{cm}^{2}, P<0.001$ ), whereas $\alpha$ remained constant at $1.59 \mathrm{dyn} / \mathrm{cm}^{2}, 4 \mathrm{dyn} / \mathrm{cm}^{2}$ and $6 \mathrm{dyn} / \mathrm{cm}^{2}$ shear stimulation (red curves in the graphs on the right side of 3.15 ).

\section{RICM measurements}

In order to determine whether the continuous increase in cardiomyocytes impedance is caused by cell-substrate contact increment or is due to variations in the distance between cell membrane and the surface RICM was used as previously explained for the analysis of shear stress immediate-short term effect on monolayers. For a long-term study, eight frames were evaluated, as mentioned in section 2.4.3.2. Both cellular mean intensity and cell-substrate contact area were normalised for their respective values at $1 \mathrm{~h}$ before the shear stress of $4 \mathrm{dyn} / \mathrm{cm}^{2}$ was applied. Figure 3.16 shows the mean time course of the normalised intensity of cells up to $19 \mathrm{~h}$ of flow stimulation. Except for the sharp decrease at the flow onset the intensity remains constant within the error limits without diverging from the initial value. This indicates that the cell-substrate distance does not change following $1 \mathrm{~h}$ of flow exposure.

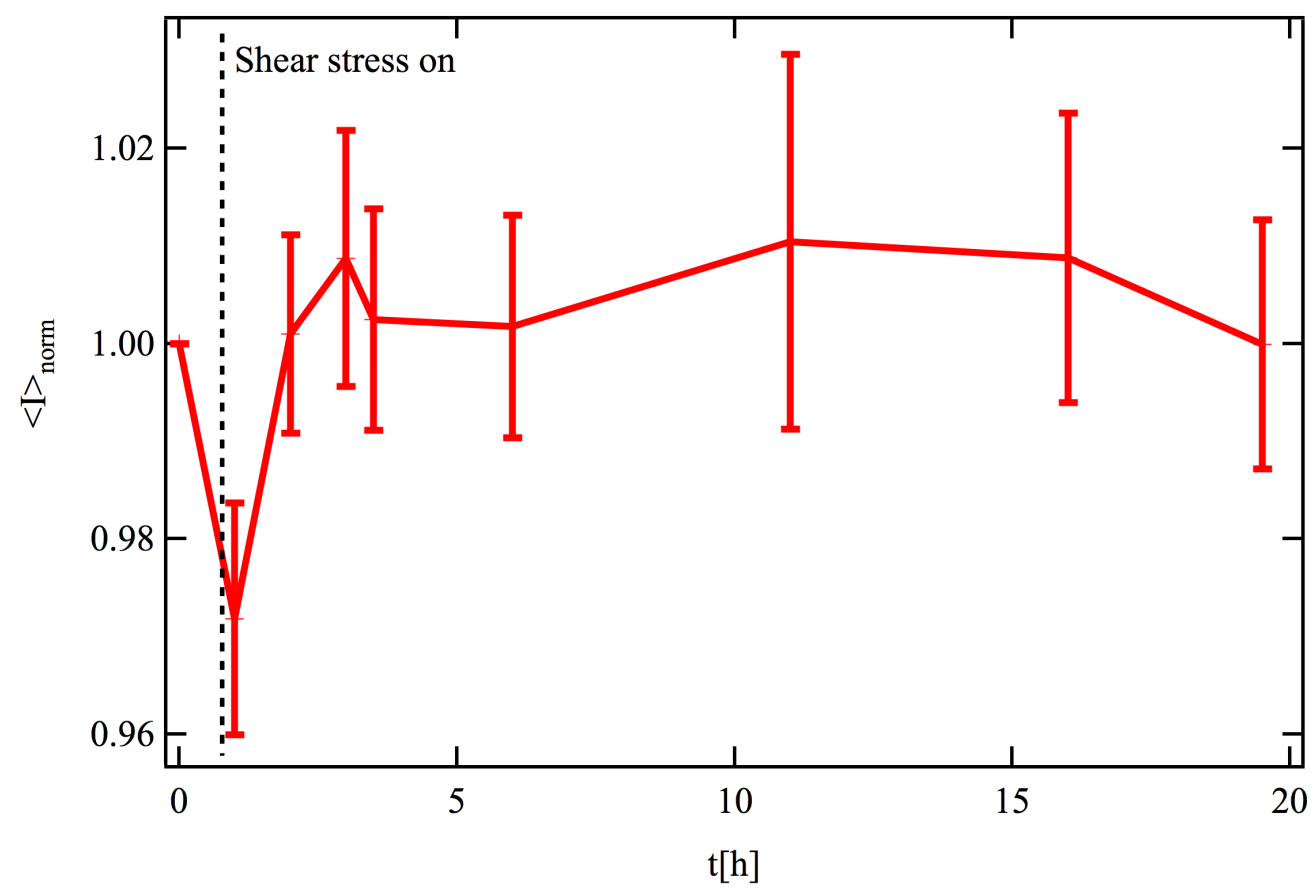

Figure 3.16: RICM intensity in the first $19 \mathrm{~h}$ of flow stimulation. Normalised cellular mean intensity for $\mathrm{n}=6 \pm$ s.d. of cardiomyocytes exposed to $4 \mathrm{dyn} / \mathrm{cm}^{2}$-shear stress. No long-term variations in RICM intensity due to the effect for the flow are observed.

On the other hand, cell-substrate contact area starts to increase approximately $2 \mathrm{~h}$ after the flow onset and continues to increase for $19 \mathrm{~h}$, as shown in figure 3.17, together with the real part of the impedance for $4 \mathrm{dyn} / \mathrm{cm}^{2}$. The time course of the mean contact area reached a 2 fold value at $19 \mathrm{~h}$ of shear stimulation and continued to slightly increase 
until $40 \mathrm{~h}$. The two grey scale micrographs, shown in figure 3.17, were recorded before and $19 \mathrm{~h}$ after the flow onset and depict the clear increase in cell contact area. It was observed that after $19 \mathrm{~h}$ cells form more and larger focal adhesions in response to the shear stress as clearly visible in the left micrograph.
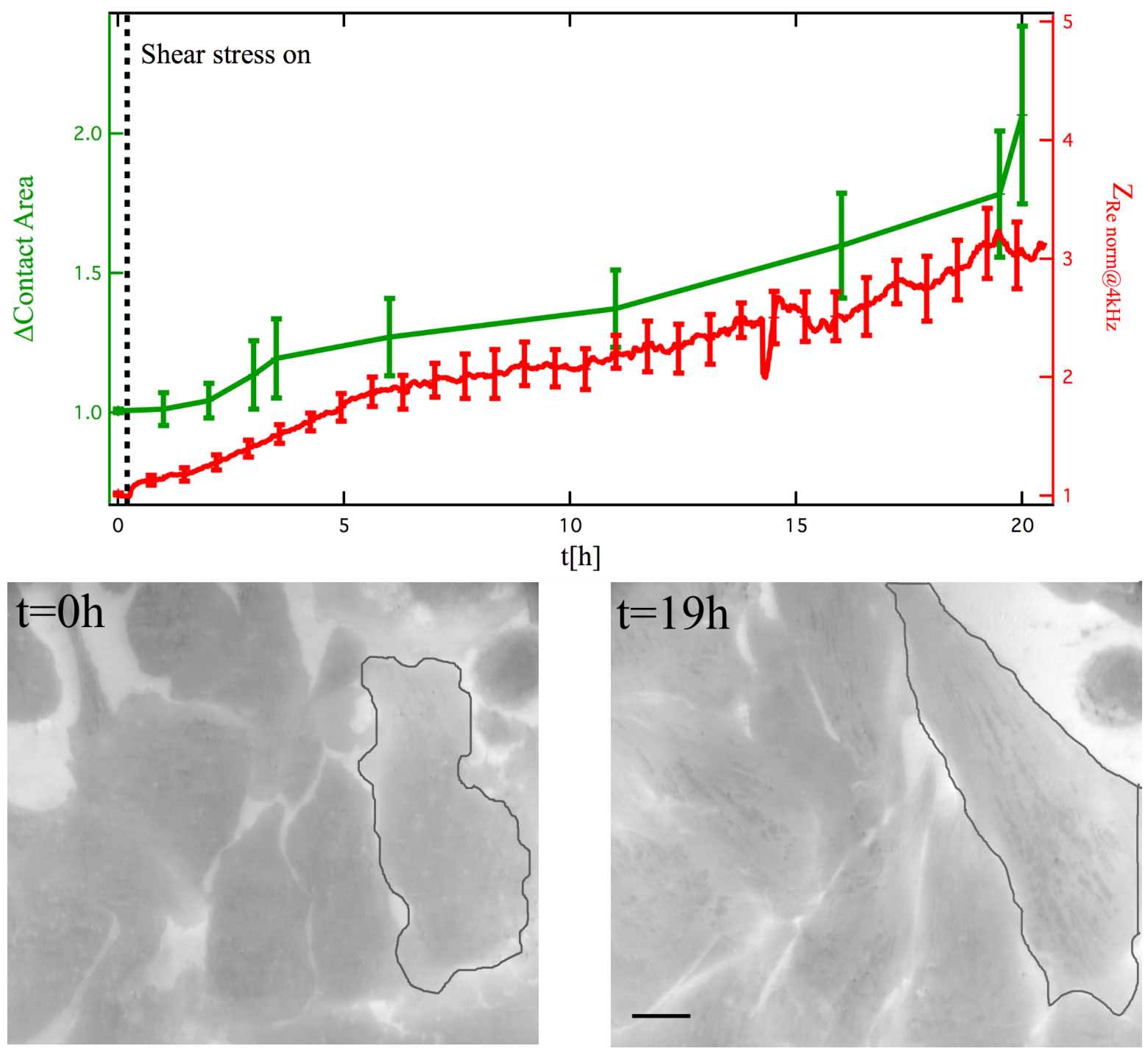

Figure 3.17: Effects of $19 \mathrm{~h}$ for shear stress on cardiomyocytes impedance and cell-substrate contact area. Top: Time course of cell-substrate contact area increment (green) and real part of the impedance (red) for a cardiomyocyte monolayer under $4 \mathrm{dyn} / \mathrm{cm}^{2}$ shear stress. Data are shown as mean \pm s.d. Bottom: RICM images of cardiomyocytes before and after shear stress was applied. $19 \mathrm{~h}$ of flow stimulation induce an increase in cell-substrate contact area and focal adhesion formation. Scale bar $10 \mu \mathrm{m}$.

These results suggest that both cell-substrate distance and contact area contribute to the increase in monolayer impedance under shear stress, but their influence does not occur simultaneously. First, the cells approaches the surface when the flow is applied subsequently returning to their original height within $1.5 \mathrm{~h}$. Afterwards, the contact area increases and the cells form larger focal adhesions. 


\section{CLSM based fiber orientation analysis}

In order to determine the effect of shear stress on cell cytoskeleton, fluorescence confocal images, recorded in at least eight different regions of $\mu$-Slide VI ${ }^{0.4}$ Luer, comparably with the position of the electrodes in the ECIS array. These images were analysed with a custom-written MATLAB program that quantifies the orientation of the actin fibers, as explained in chapter 2.4.1.3. Cardiomyocytes exposed to shear stress of $4 \mathrm{dyn} / \mathrm{cm}^{2}$ for $108 \mathrm{~h}$ are horizontally aligned and elongated in the direction of the flow (horizontal axis). By contrast, the static-cultivated cells are randomly distributed without any
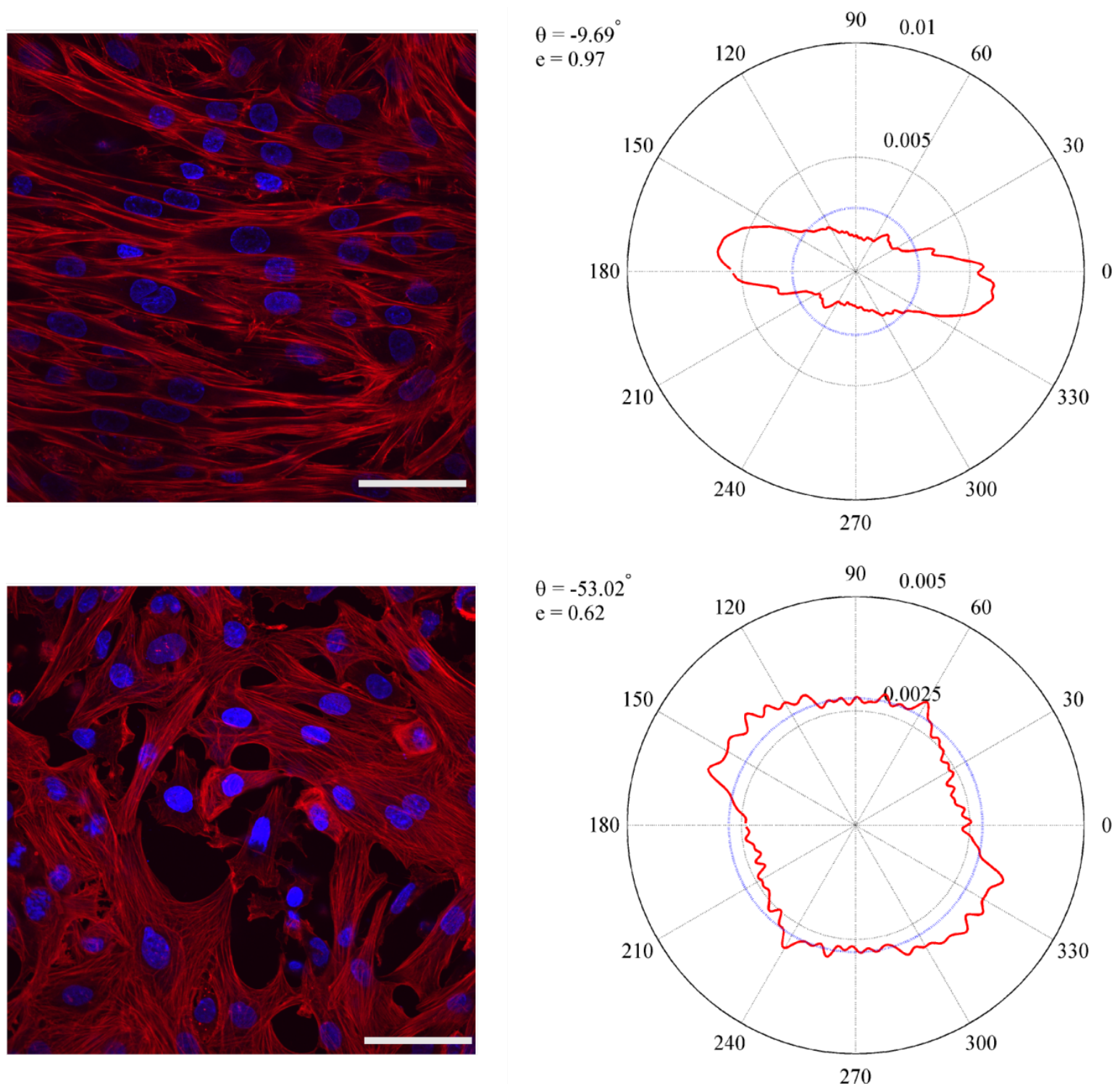

Figure 3.18: Exemplary confocal images of actin fibers of cells exposed to $4 \mathrm{dyn} / \mathrm{cm}^{2}$ shear stress for $108 \mathrm{~h}$ (Top) and of cells cultivated under static conditions (Bottom). For both frames, the normalized fiber orientation distribution in polar coordinates, the eccentricity $e$ of the fitted ellipse and the angle of major axis $\theta$ are shown. Scale bar $80 \mu \mathrm{m}$. Actin fibers are stained with Alexa-Fluo-488-phannotoxins (red) and nuclei with DAPI (blue). 
preferential direction. Analysis by the program which uses a 2D-FT yields an orientation factors $e . e$ is the eccentricity of an ellipse that fits the normalised fiber orientation distribution in polar coordinates and describes that $67 \%$ of the fibers are oriented within a certain angle. If this angle is large then the orientation is random, whereas if it is small, the fibers are along one preferential direction. The $e$ values extracted from the images of stimulated and non-stimulated monolayers clearly showed that the fibers were preferentially oriented and randomly distributed, respectively. In figure 3.18, one exemplary evaluation for cells sheared for $108 \mathrm{~h}$ and for cells under static conditions with their respective plot of the fibers orientation distribution (red) are shown. The light blue circle represents the distribution of an image with completely random orientations of fibers. $\theta$ is the angle along which the largest number of fibers is distributed. Flow exposed cardiomyocites showed $e=0.97, \theta=-9.7^{\circ} \mathrm{C}$ and an elongated fiber orientation distribution, whereas for not-sheared cell image $e=0.62, \theta=-53^{\circ} \mathrm{C}$ and the distribution is close to a randomly oriented distribution.

The spatial distribution of actin fibers over time was obtained by comparing images of sheared and non-sheared cells after $0 \mathrm{~h}, 48 \mathrm{~h}, 72 \mathrm{~h}$ and $108 \mathrm{~h}$ of flow stimulation. Exemplary fluorescence images are show in figure 3.19, together with a bar plot of the eccentricity over time. Only images of cardimyocytes exposed to shear stress for $108 \mathrm{~h}$ exhibit a normalized fiber orientation distribution with eccentricity higher than 0.9 , meaning that the $67 \%$ of the fibers are oriented within an angle $\theta_{\text {err }} \leq 37^{\circ}$. The $e$ values increase with the duration of flow exposure indicating a gradual increase of the orientation of the actin fibers. Also the difference between the eccentricity of sheared and non-sheared normalized fiber distribution increases when the flow stimulation last for longer time. These results show that under shear stress the actin fibers gradually orient over time and after $108 \mathrm{~h}$ more than $67 \%$ are aligned in the direction of the flow. 


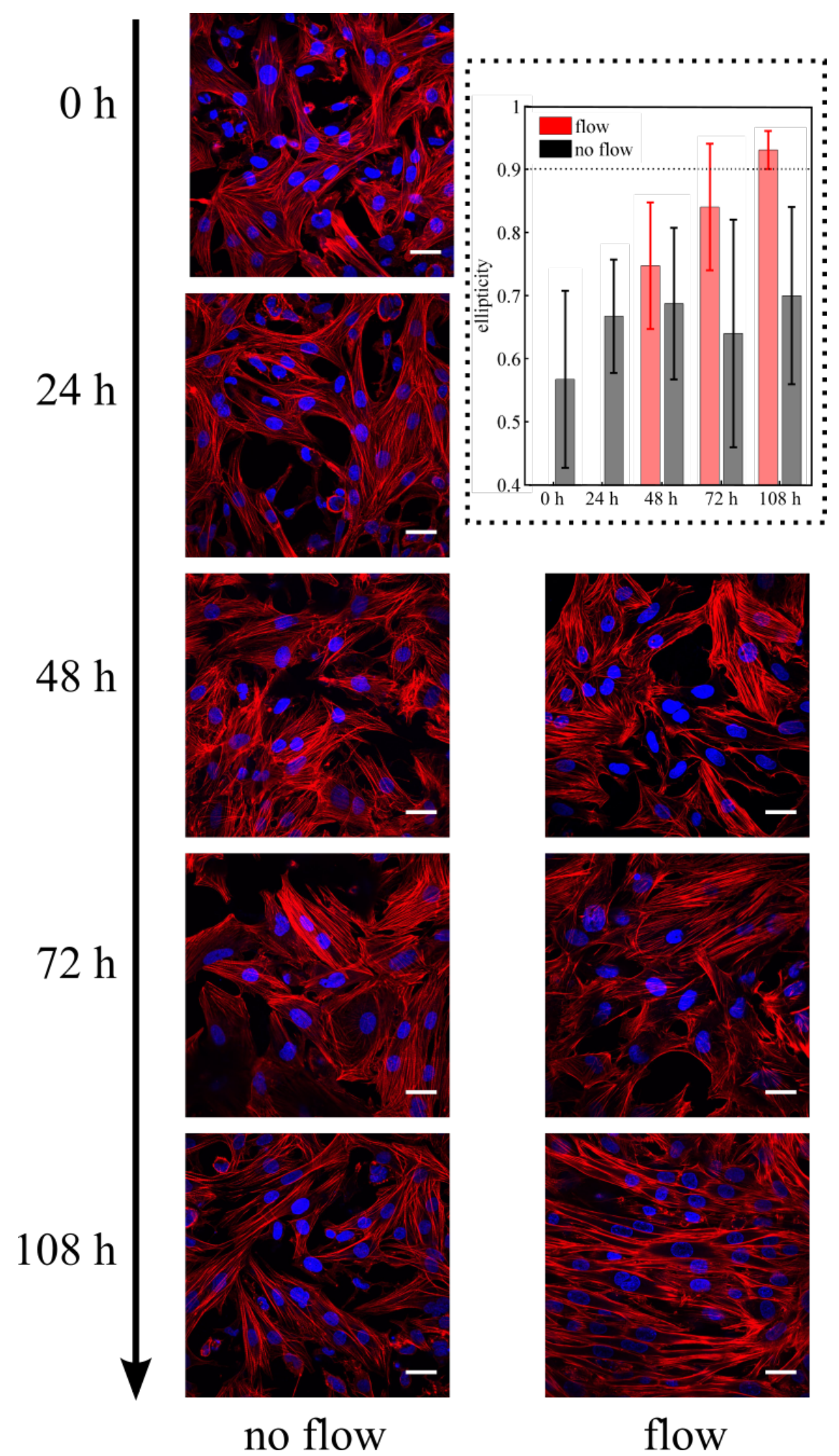

Figure 3.19: Time sequence of confocal images of cells exposed to shear stress for $48 \mathrm{~h}, 72 \mathrm{~h}, 108 \mathrm{~h}$ and non-sheared cells. Scale bar $50 \mu \mathrm{m}$. Bar plot of the eccentricity $e$ of the normalized fiber orientation distribution. When $e=0$, fibers are randomly oriented, while $e=1$ fibers are perfectly aligned with the direction of the ellipse major axis. In this work, it was considered that fibers $(67 \%)$ were well oriented only if $e>0.9$. Data are shown as mean of at least $n=8 \pm$ s.d. 


\subsubsection{Long term effect of shear stress on cellular contractility}

High sampling impedance measurements were performed to investigate the influence of fluid stimulation for $108 \mathrm{~h}$ on cardiomyocytes monoculture and cadiomyocytes/fibroblast co-culture beating frequency.

\section{Cardiomycyte monocultures}

Impedance fluctuations due to cell contractility were recorded every $24 \mathrm{~h}$ sequentially for each cell covered electrode inside the ECIS Flow Array before the medium was exchanged. Impedance power spectral density (PSD) analysis shows that the effect of shear stress induces changes in beating frequency of cells. When a stress of $1.59 \mathrm{dyn} / \mathrm{cm}^{2}$ is continuously applied for $24 \mathrm{~h}$, cardiomyocytes beating rate slightly increases (1.2) and after $48 \mathrm{~h}$ of stimulation it reaches a value of 1.4 times higher than the value of non sheared cells $(P<0.05)$. In the following $60 \mathrm{~h}$, the beating frequency decreases gradually until the end of the experiment (figure 3.20). When higher shear stresses of $4 \mathrm{dyn} / \mathrm{cm}^{2}$ and $6 \mathrm{dyn} / \mathrm{cm}^{2}$ were applied for $24 \mathrm{~h}$ cells were beating faster than the non-stimulated ones by a factor 1.8 and 1.7, respectively. Similarly to $1.59 \mathrm{dyn} / \mathrm{cm}^{2}$, under higher shear stresses the beating frequency reaches a maximum at $48 \mathrm{~h}$ after the flow onset corresponding to $2.79 \mathrm{~Hz}$ for $4 \mathrm{dyn} / \mathrm{cm}^{2}$ and $2.99 \mathrm{~Hz}$ for $6 \mathrm{dyn} / \mathrm{cm}^{2}$. In table 3.3 values of beating frequencies before $(\mathrm{t}=0)$ and after $24 \mathrm{~h} / 48 \mathrm{~h}$ of flow exposure to different shear stresses are listed ( \pm s.d.). By applying higher values of shear stress to cardiomycytes monolayer an increase of the beating rate was observed. This increment appears lin-

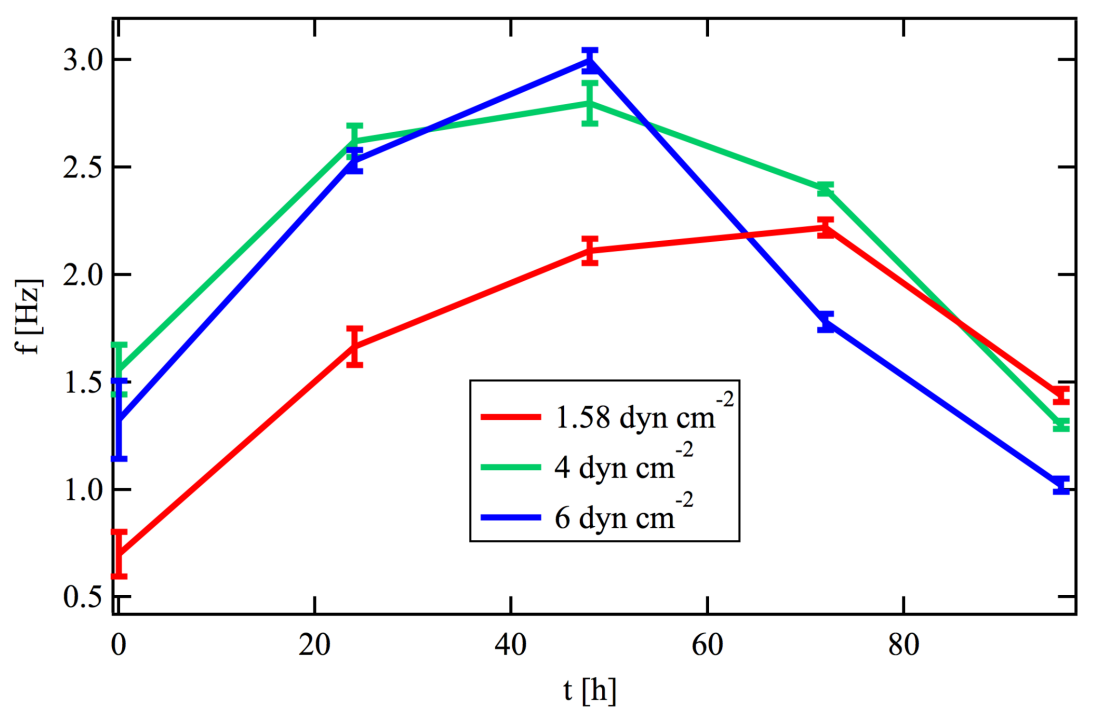

Figure 3.20: Beating frequency of cardiomyocytes sheared with $1.59 \mathrm{dyn} / \mathrm{cm}^{2}$ (red) ( $n=7$ electrodes), $4 \mathrm{dyn} / \mathrm{cm}^{2}$ (green) ( $n=7$ electrodes) and $6 \mathrm{dyn} / \mathrm{cm}^{2}$ (blue) $(n=8$ electrodes) over time. A peak in beating frequency occurs $48 \mathrm{~h}$ after flow onset with shear stresses of $4 \mathrm{dyn} / \mathrm{cm}^{2}$ and $6 \mathrm{dyn} / \mathrm{cm}^{2}$. For cells sheared at $1.59 \mathrm{dyn} / \mathrm{cm}^{2}$ there is no significant difference between beating frequency at $\mathrm{t}=48 \mathrm{~h}$ and $\mathrm{t}=72 \mathrm{~h}$. Data are presented as mean \pm s.d. 
ear until shear stress of $4 \mathrm{dyn} / \mathrm{cm}^{2}$ is applied and a further increase in the amount of stimulation does not leads to a significant increase in contractility $\left(6 \mathrm{dyn} / \mathrm{cm}^{2}\right)$. The presence of fluid flow reduced the variability in the beating frequency in the cell population along the channel, as can be seen from the value of standard deviations. This suggests that the fluid flow aids the cells to synchronise their beating. These results are shown in figure 3.21, displaying the peak of beating frequency, occurring after $48 \mathrm{~h}$ of stimulation, as a function of shear stress.

\begin{tabular}{cccc}
\hline $\begin{array}{c}\text { Shear stress } \\
{\left[\mathrm{dyn} / \mathrm{cm}^{2}\right]}\end{array}$ & $\mathbf{t}=\mathbf{0} \mathbf{h}$ & $\mathbf{t}=\mathbf{2 4} \mathbf{h}$ & $\mathbf{t}=\mathbf{4 8} \mathbf{h}$ \\
\hline 0 & $0.74 \pm 0.38$ & $1.42 \pm 1$ & $1.66 \pm 0.53$ \\
1.59 & $0.69 \pm 0.10$ & $1.66 \pm 0.08$ & $2.11 \pm 0.05$ \\
4 & $1.55 \pm 0.11$ & $2.60 \pm 0.07$ & $2.79 \pm 0.09$ \\
6 & $1.39 \pm 0.18$ & $2.53 \pm 0.05$ & $2.99 \pm 0.05$ \\
\hline
\end{tabular}

Table 3.3: Values of cardiomyocytes beating frequency before $(\mathrm{t}=0)$ and after $24 \mathrm{~h}$ and $48 \mathrm{~h}$ fluid stimulation was applied. Data are presented as mean \pm s.d.

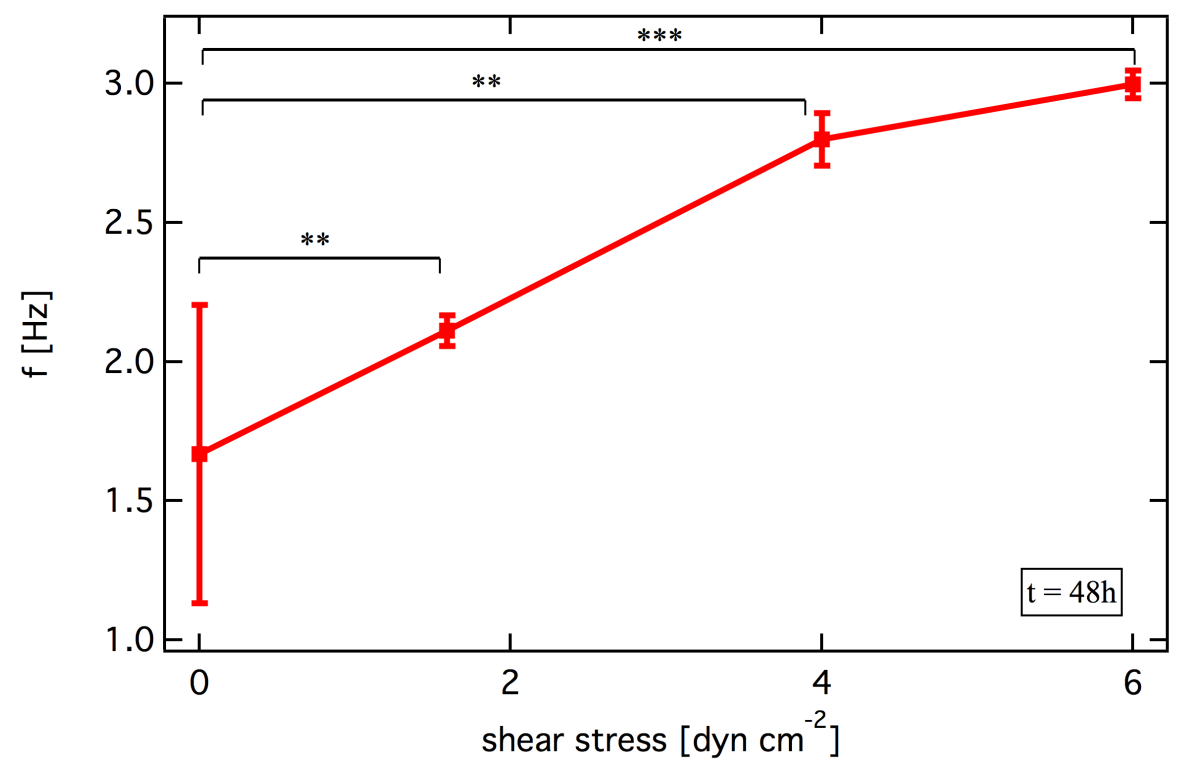

Figure 3.21: Cardiomyocytes monoculture frequency as function of shear stress after $48 \mathrm{~h}$ of stimulation. Data are presented as mean \pm s.d., $1.59 \mathrm{dyn} / \mathrm{cm}^{2} \mathrm{n}=7,4 \mathrm{dyn} / \mathrm{cm}^{2} \mathrm{n}=7,6 \mathrm{dyn} / \mathrm{cm}^{2} \mathrm{n}=8$, no flow $\mathrm{n}=5$. Significant difference was observed between static culture and $1.59 \mathrm{dyn} / \mathrm{cm}^{2}$ sheared cells, $4 \mathrm{dyn} / \mathrm{cm}^{2}$ (Wilcoxon Rank Test, $P<0.05$ ) or $6 \mathrm{dyn} / \mathrm{cm}^{2}$ (T test, $P<0.001$ ). Statistical differences were also observed between $1.59 \mathrm{dyn} / \mathrm{cm}^{2}, 4 \mathrm{dyn} / \mathrm{cm}^{2}, 6 \mathrm{dyn} / \mathrm{cm}^{2}$ (T test, $P<0.001$ ). An increase of applied shear stress induces an increase in beating frequency.

\section{Cardiomycyte/fibroblast co-cultures}

The effect of flow on the beating frequency in co-cultures with different percentage of fibroblasts were studied similar to cardiomyocytes monocultures by monitoring the im- 
pedance fluctuations. The highest value of shear stress previously used, $6 \mathrm{dyn} / \mathrm{cm}^{2}$, was chosen for stimulating the co-cultures. Contactility was analysed $48 \mathrm{~h}$ after the flow onset that corresponds to the time at which the beating frequency of cardiomyocytes monocultures reached the maximum. Figure 3.22 shows the mean value of beating frequency of the cardiomyocytes/fibroblasts co-cultures with and without flow exposure as function of the ratio of the cells. When no flow was applied and the percentage of cardiomyocytes in the sample was reduced from $100 \%$ to $25 \%$, the beating rate of the co-cultures decreases up to the disappearance of contractile behaviour at $10 \%$ cardiomyocytes ratio. There is no significant difference in the beating properties of nonstimulated $50 \%$ and $25 \%$ cardiomyocytes-fibroblasts ratios. During exposure to shear stress the beating rate increases for all the cardiomyocytes/fibroblasts mixtures similar to the cardiomyocytes monocultures. The frequency for samples with $100 \%$ cardiomyocytes, $50 \%$ cardiomyocytes and $25 \%$ cardiomyocytes is $1.8,1.6$ and 2 times the values of the controls, respectively. However, no significant difference between the beating rates of stimulated $50 \%$ fibroblasts and $25 \%$ cardiomyocytes was observed which was confirmed with a statistical t-test. Surprisingly, co-cultures with only $10 \%$ cardiomyocytes show

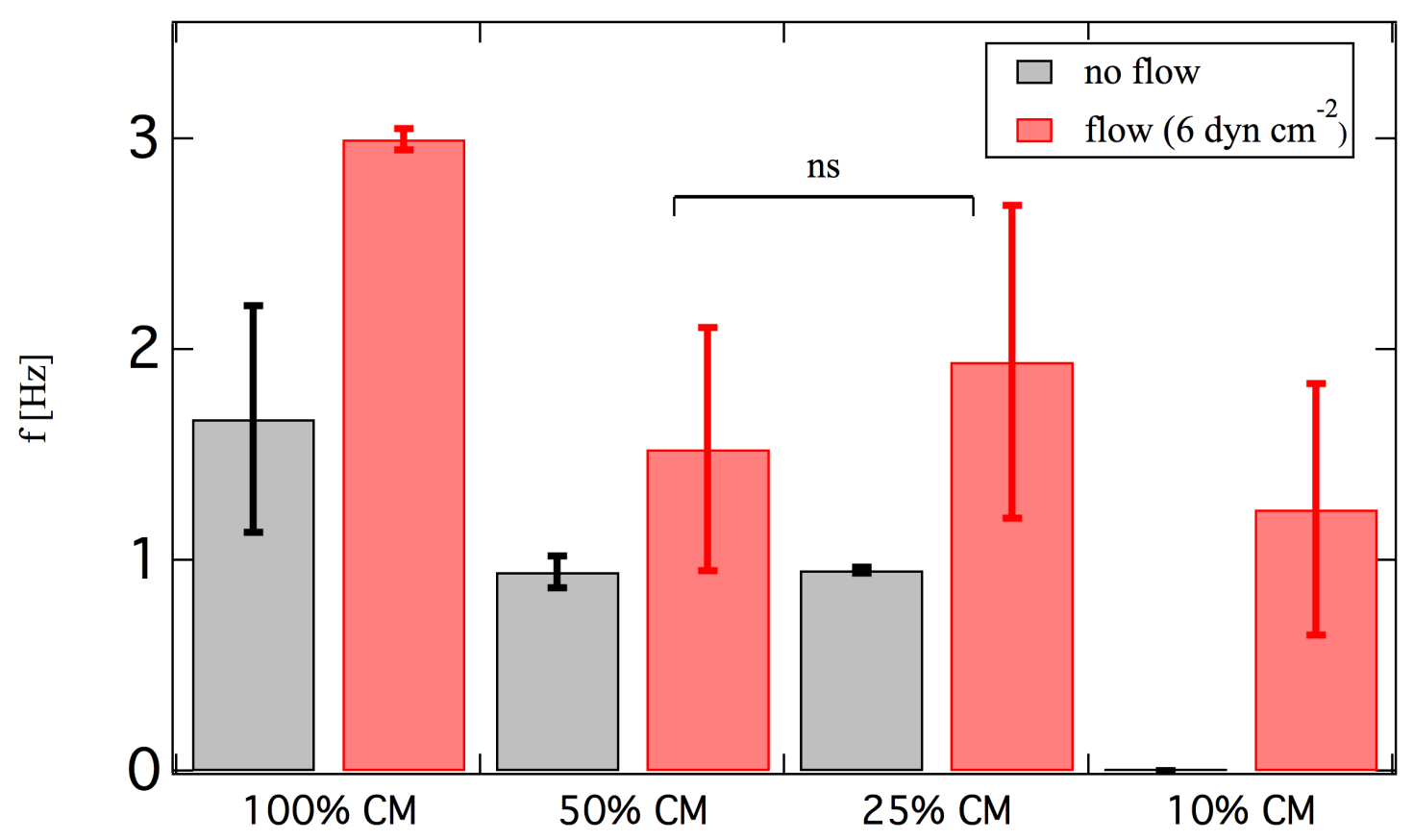

Figure 3.22: Beating frequency of co-culture varying with the percentage of cardiomyocytes (CM) and fibroblasts under static and sheared conditions $\left(6 \mathrm{dyn} / \mathrm{cm}^{2}\right)$. Data are shown $48 \mathrm{~h}$ after flow onset as mean \pm s.d. Increased beating frequency of cells exposed to flow stimulation was observed for every cardiomyocytes/fibroblasts mixture, with no significant difference between $50 \%$ cardiomyocytes and $25 \%$ cardiomyocytes. In the case of $10 \%$ cardiomyocytes, spontaneous beating was observed exclusively when shear stress was applied. 
a contractile behavior with beating frequency of $1.2 \mathrm{~Hz}$ after being exposed to $48 \mathrm{~h}$ at $6 \mathrm{dyn} / \mathrm{cm}^{2}$ shear stress.

To summarise, the exposure to shear stress induces an increase of the beating frequency in cardiomyocytes/fibroblasts co-cultures independent of the percentage of cardiomyocytes and may promote the transformation of fibroblasts into myofibroblasts. 


\section{Discussion}

The aim of this work was to study the left ventricular remodeling at cellular level, that occurs typically after acute myocardial infarction (MI). MI usually results in generation of arrhythmia and can cause often heart failure and lead to death. Post-infarction remodeling can be divided into two phases: early phase, within 72 hours, and late phase [3]. The early phase involves cardiomyocytes slippage, which leads to thinning and dilatation of the myocardial wall, interstitial growth, and myocytes lengthening (hypertrophy), whereas the late phase is characterised by a recruitment of fibroblasts in the infarcted area and their transformation into contractile myofibroblasts. Concurrently, an excessive synthesis of collagen also takes place, which leads to the scar tissue formation (fibrosis). This stabilises the increased wall stress, caused by the ventricle dilatation. Current therapies targeting the post-infarction remodelling are based on mechanical or pharmacological interventions, but both present drawbacks. Therefore, it is necessary to understand deeply the remodelling mechanisms to develop new targeted therapeutic strategies. It is essential to investigate the pathological state of cardiomyocytes by considering also the biophysical stimuli the cells are subjected to. Therefore, I investigated the effect of particular conditions, such as high levels of shear stress and different degrees of fibrosis that occur in the early and late phase of ventricular remodelling, on the cellular morphology, contractility, connectivity, and mechanical properties.

\subsection{Ventricular Remodeling: Fibrosis}

The development of cardiac fibrosis that eventually leads to arrhythmia is thought to be strongly related to the communication between cardiomyocytes and fibroblasts. Among the several mechanisms of myocyte-fibroblast interaction, I focussed on the direct cross-talk between cells through electrical and mechanical junctions and its variation with an increasingly severe fibrosis. To engineer an in vitro cardiac fibrosis model, I used co-cultures of primary neonatal cardiomyocytes and heart-derived fibroblasts. Cocultures in which fibroblasts expressed the contractile protein $\alpha$-smooth muscle actin $(\alpha$-SMA) within 5 days were referred to as fibrotic monolayers. The presence of $\alpha$-SMA 
was indicative of the transformation of fibroblasts into myofibroblasts that appear in vivo only after cardiac injury and that are critical components of cardiac fibrosis [57, 58]. Different ratios of fibroblasts were added to the co-cultures to mimic different degrees of fibrosis. The relative occurrence of homotypic and heterotypic junctions in the fibrotic monolayers was quantified by staining the gap (electrical) and adherens (mechanical) junctions. Gap junctions, depicted by the localisation of Connexin 43 (Cx43), were observed between all cell types in all the co-cultures with different cardiomyocytemyofibroblast ratio. In a healthy heart the electrical signal is transmitted through the myocardium solely by the cardiomyocytes via $\mathrm{Cx} 43$, whereas after a cardiac injury myofibroblasts express high levels of Cx43 as well [59]. This is consistent with the outcomes of this work, as described in section 3.1.1.1, which show the increase in myofibroblasts homocellular gap junctions at low cardiomyocytes density in the co-cultures. Pedrotty et al. have reported that micro-patterned homotypic pairs of myofibroblasts express Cx43 mainly inside the cytoplasm rather than at the cell-cell junctions $(<10 \%$ of the 450 studied cell pairs) [60]. With the analysis routine, described in section 2.4.1.2 it was not possible to discern whether the stained protein was located in the intracellular space or at the junction between the same type of cells. This uncertainty can be overcome by considering the cellular barrier resistance $R_{b}$, which quantifies the cell-cell connectivity within the fibrotic monolayer and was observed to increase in co-cultures with lower ratio of cardiomyocytes as shown in figure 3.4. It reached a maximum for $10 \%$ of the cardiomyocytes in co-culture indicating that significant amount of $\mathrm{Cx} 43$ is localised in myofibroblasts homocellular junctions. Nevertheless, the presence of Cx43 in the cytoplasm cannot be excluded. Indeed it can be the newly expressed Cx43, known to contribute to TGF- $\beta$ signalling to regulate $\alpha$-SMA expression [61], is indicative of the transformation of fibroblasts into myofibroblasts as mentioned above. Western blot assay is a possible technique to quantify the amount of junctional vs cytosolic Cx43 [61].

An enriched Cx43 expression by myofibroblasts in fibrotic conditions leads to a higher possibility for cardiomyocyte-myofibroblast coupling. Myofibroblasts are known to be unable to generate action potential but they posses conductive properties. They are characterised by a high membrane resistance and a hyperpolarized membrane potential and they depolarise cardiomyocytes by imposing their electrical load when paired to them through gap junctions [62]. The observation of spiral waves in cardiomyocytesmyofibroblast co-cultures, as shown in figure 3.6, indicates the influence of myofibroblasts on the electrophysiology of cardiomyocytes through electrical and mechanical coupling. Further, the coupling between cardiomyocytes and myofibroblasts results in a decrease in the beating frequency of the co-cultures. This can be seen from figure $3.4 \mathrm{~B}$, which shows a gradual decrease in the beating frequency with the increase of the myofibroblasts ratio. Numerous studies support this findings which show that an 
increased myofibroblast-cardiomyocyte coupling decreases the action potential duration and slow conduction velocity [63] that leads to arrhythmia [64, 65]. The results in section 3.1.2 shows that spontaneous beating was observed in co-culture with more than $25 \%$ cardiomyocytes, indicating that there exists a maximum distance between two cardiomyocytes linked by a bridge of myofibroblasts above which the transmission of action potential is hindered. This is confirmed by a quantitative study which shows that the impulse propagation between distant cardiomyocytes through myofibroblasts occurs up to a distance of $300 \mu \mathrm{m}$ [66]. An additional type of cell-cell connection that may affect the increase in the cellular barrier resistance $R_{b}$ of fibrotic monolayers are membrane nanotubes. These are long and thin membrane connections identified in cardiomyocytes and myofibroblasts that permit long-distance transfer of membrane components, mitochondria, and $\left[\mathrm{Ca}^{2+}\right]$ [67]. The possibility that these structures could be involved in communication between cardiomyocytes and myofibroblasts in fibrotic condition is not clarified.

Adherens junctions also contribute to the increase in barrier resistance $R_{b}$ of fibrotic monolayers. Similar to $\mathrm{Cx} 43$, N-cadherin (N-Cad) was also localised between cardiomyocytes, myofibroblasts and cardiomyocyte-myofibroblast pairs, suggesting the direct mechanical interaction between both cell types. High fibroblast homocellular adherens junctions were also observed in co-cultures with low cardyomyocyte ratios, as shown in figure 3.2. It is reasonable to think that high density of junctional N-Cad is required to create solid mechanical adhesions where overexpressed $\mathrm{Cx} 43$ can localise. Studies of cardiac development of isolated adult cardiomyocytes, have shown that adherens junctions, which bind to the plus end of the microtubules in the intercalated disks, are formed before Cx43 is transported via microtubules to the cell membrane to constitute gap junctions [68]. I speculate that the occurrence of both gap and adherens junctions between myofibroblasts in fibrotic monolayers with lower percentage of cardiomyocytes in my experiments is due to this sequential expression and localization of the junction proteins. Moreover, the results in section 3.1.1.2 show that the homocellular $\mathrm{N}$-Cad mediated junctions between myofibroblasts increased exponentially with the ratio of myofibroblasts, compared to the gradual increase of gap junctions. The different expression levels of $\mathrm{N}$-Cad and $\mathrm{Cx} 43$ suggests that myofibroblasts form strong mechanical junctions than electrical coupling. This might indicate that myofibroblasts prefer cell-cell communication through contractile forces rather than electrical signals. Consequently, it is reasonable to hypothesise that myofibroblasts use mechanical feedbacks on cardiomyocytes as well. Interestingly, in this work it was observed that myofibroblasts physically deform the membrane of cardiomyocytes through N-Cad junctions (figure 3.2), suggesting the possibility that myofibroblasts exert forces both on myofibroblasts and cardiomyocytes, probably modulating their performance. The idea 
that myofibroblasts can affect electrophysiology of cardiomyocytes via mechanical interactions was also confirmed by Thompson et al. In their study they show that the conduction velocity in co-cultures with prevalent heteroadhesion junctions can be fully restored by applying contraction blockers (e.g. Blebbistatin) or mechanosensitive channel blockers [63]. Another study observed that pharmacological ablation of $\alpha$-SMA nullify arrhythmogenic myofibroblasts-cardiomyocytes communication [69]. Following this observation, I speculate that the increased homocellular mechanical and electrical junctions between myofibroblasts in increasingly severe fibrosis might lead to disturbance in conduction and arrhythmia. In summary post-infarct area cannot be considered as a passive tissue. The assumption that myofibroblasts could act exclusively as insulator after MI is obsolete. Fibroblasts can transform into contractile myofibroblasts, couple mechanically and electrically with cardiomyocytes, and affect cardiac electrophysiology. In figure 4.1 I summarise a scheme the electromechanical crosstalk between cardiomyocytes and myofibroblasts under fibrotic conditions analysed in this work.

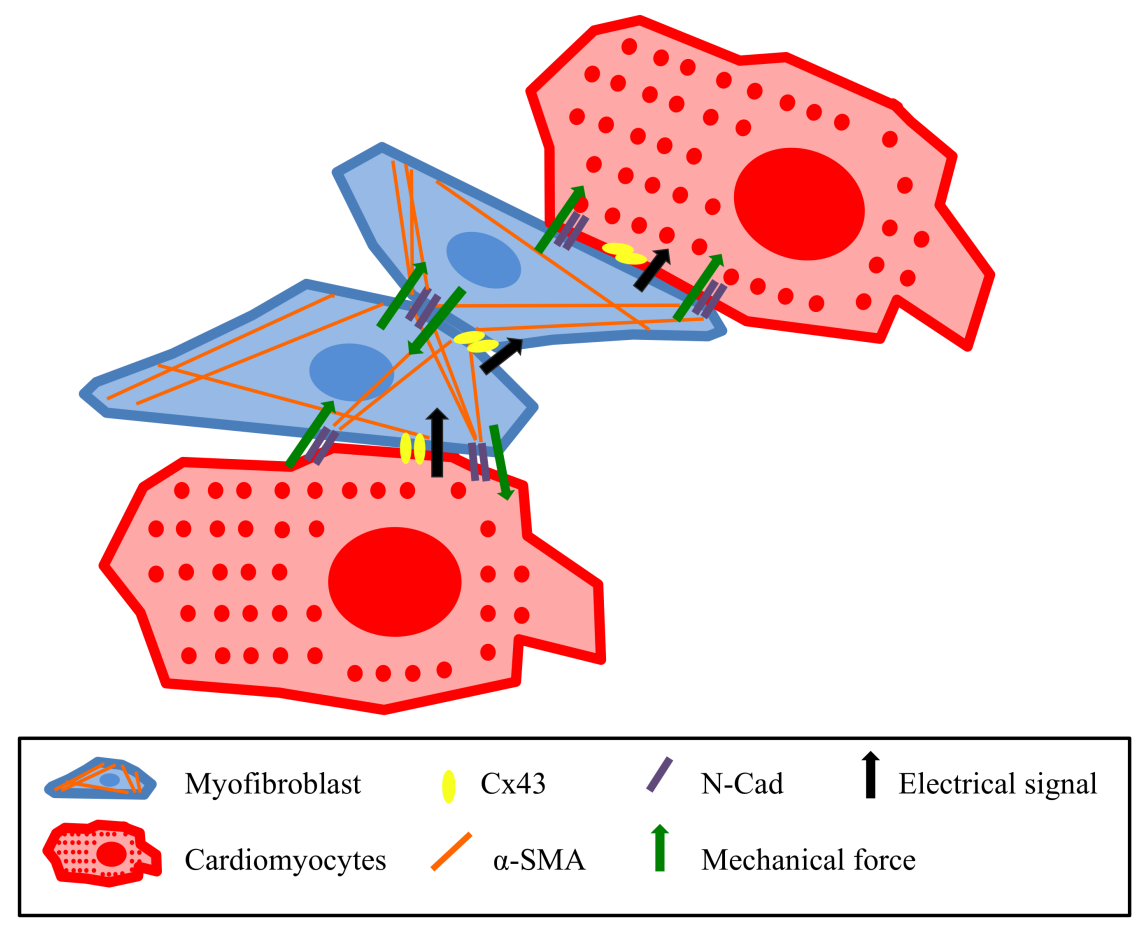

Figure 4.1: Mechanoelectrical crosstalk between cardiomyocyte and myofibroblasts.

Apart from this direct type of communication, it is known that cardiomyocytes and fibroblasts indirectly interact releasing chemical mediators as TGF- $\beta 1$ and Angiotensin II or using ECM proteins as mediators [16]. During fibrosis an overproduction of ECM components, e. g. collagen and fibronectin, due to the hyperactivity of myofibroblasts results in an increased stiffness of the infarcted region that affect the surrounding cardiomyocytes. Studies have shown that alterations in collagen levels were associated with damaged cardiomyocytes connectivity and function $[16,70]$. Therefore, it is essential to 
investigate the mechanical properties of cells under in vitro fibrotic conditions. In section 3.1.3 I characterised the viscoelastic properties in monocultures of cardiomyocytes and fibroblasts in comparison to fibrotic co-cultures by using AFM-based microrheology following the method introduced by Shroff and coworkers and modified by Alcaraz et al $[51,52]$. Among other microrheological techniques AFM has the highest lateral resolution and allows the determination of both solid- and fluid-like features of cardiac monolayers. Cellular mechanical response was characterised by extracting $G^{\prime}$ and $G^{\prime \prime}$, accounting for the stored elastic energy and the frictional energy dissipated, respectively, from oscillatory indentation measured at different frequencies. A simultaneous increase of the $G^{\prime}$ and $G^{\prime \prime}$ with increasing frequency was observed for cardiomyocytes, no-activated fibroblasts, and fibrotic co-culture (equal density of cardiomyocytes and myofibroblasts), indicating a frequency dependence of the viscoelastic properties of the cells. This is similar to the previous studies on rheological properties of different cell types [52]. Loss tangent $\eta$, which is the ratio between $G^{\prime}$ and $G^{\prime \prime}$ and is indicative of the transition from solid-like $(\eta<1)$ to fluid-like $(\eta>1)$ behaviour, also increases with high frequencies. However, the relation between frequency and $\eta$ shows a different trend according to the population ratio in the co-culture, as shown in figure 3.8. Cardiomyocytes show prominent elastic behaviour at frequencies smaller than $80 \mathrm{~Hz}$ whereas for fibroblasts the solid-fluid transition occurs already at $60 \mathrm{~Hz}$. The more fluid-like nature of the fibroblasts is probably the reason why these cells migrate in the damaged area after cardiac injury to start wound healing process. Such a migration implicates that they are subjected to numerous deformation, e.g squeezing between cardiac sheets to reach the target area.

The results obtained in section 3.1.3 show that fibrotic conditions lead to a general decrease of $\eta$, indicating that the co-culture behaves rather as a solid with fluid-like transition shifted at high frequencies, congruent with a typical increase in stiffness that characterises fibrosis. The frequency dependent rheological results can be explained by the power-law structural damping model, that Fabri and coworkers interpreted as a particular case of soft glassy rheology [71]. According to the model, the cellular cytoskeleton is a network of structural elements not permanently attached to each other, weakly interacting and trapped in energy wells. Perturbation of the network, such as external forces or active motion induced by motor proteins, provide energy to the system causing a jump to other energy wells and a shift to either more fluid or more solidlike state. The power-law coefficient represents a sort of temperature of the material indicating the level of agitation in the network. Cardiomyocyte monocultures exhibit a slightly higher power-law coefficient (0.35), although possessing an elevated concentration of proteins important for force generation and contraction, especially $\alpha$-actin and myosin. These contraction proteins form well organised myofibrils and maintain 
cellular structure elements at their positions, preventing jumps from energy wells. For such a configuration the power-law coefficient is supposed to approach zero. However, cardiomyocyte contractility is associated with cytoskeleton rearrangements and active processes that involve energy consumption, and increase in this way the network agitation. Rheological measurements also show that cardiomyocytes monocultures are stiff and this is attributable to the elevated concentration of proteins involved in the process of contraction, as mentioned above. On the contrary, fibroblasts monocultures are 1.5 time softer and exhibit lower viscosity and lower power-law coefficient compared to cardiomyocytes. This is easy to understand considering the fact that fibroblast are cells involved in migratory processes, such as wound healing, and that migration is promoted by an increase of cytoskeletal tension [72]. DiMilla et al. demonstrated in silico that there is a relationship between contractile force, cellular rheology and migration velocity [73]. An increased cytoskeletal tension is required to promote cellular deformation and migration [72]. It has been reported that factors that induce a decrease of power-law coefficient lead to an increase in cytoskeletal tension [74]. Additionally, a decrease in viscosity leads to an increase in migration speed. All these factors facilitate fibroblasts to reach their target in different tissues and in particular, in the case of fibrosis, the post-infarction area and then differentiate into myofibroblasts.

Fibrotic co-cultures, obtained by mixing cardiomyocytes and myofibroblasts are characterised by an increase in stiffness, $G^{\prime}, G^{\prime \prime}$, and a decrease of $\eta$, compared with both cardiomyocytes and fibroblasts. Furthermore, a decreased power-law coefficient, revealing a low cytoskeleton agitation, confirm that the co-culture appears stiffer and more like an elastic material. This indicates the fibrotic co-culture behaves rather as a solid with fluid-like transition shifted at high frequencies $(150 \mathrm{~Hz})$, and elasticity dominates over viscosity. This is congruent with the typical increase in stiffness that characterises fibrosis. Similar effects have also been observed in normal murine mammary gland (NMuMG) cells after incubation with TGF- $\beta 1$ which induces an increase of cellular elastic behaviour, stiffness and a decrease in power-law coefficient [75]. Thus, the incubation of TGF- $\beta 1$ results in a structural rearrangement of the cytoskeleton and a new formation of stress fibers near the apical membrane [75]. I assume that changes in fibroblast mechanics might be induced by TGF- $\beta 1$ that is produced by cardiomyocytes under fibrotic conditions. The TGF- $\beta 1$ is also crucial for fibroblast activation characterised by the expression of $\alpha$-SMA contractile protein. Probably the surrounding cardiomyocyes in the co-culture are also affected, resulting in an overall increase in the stiffness and a prominent solid-elastic behaviour. It should be pointed out here that the fibrotic co-cultures maintain the viscosity comparable to the cadiomyocytes and the power-law coefficient identical to the fibroblasts. My hypothesis is that they are in a sort of mechanical metastate inbetween the two type of cells. The idea that changes in viscoelastic 
properties of infarcted cardiac tissue alter the mechano-electric feedback affecting the arrhythmia onset is supported by a mathematical model introduced by Katsnelson et al. [76]. According to their model, myocardial mechanics (viscosity and elasticity) may modulate the load of Sarcoplasmic Reticulum $\left[\mathrm{Ca}^{2+}\right]$ and induce spontaneous activity of cardiomyocytes leading to cardiac rhythm disturbances. Specifically, any increase in either viscosity or elasticity of intracellular structures such as constitutive proteins (e.g. collagen), microtubules network, and actin-myosin slipping promotes arrhythmia. Several cardiac pathologies including hypoxia, anoxia, and ischemia are characterised by modified viscoelastic properties [76]. I was able to demonstrate cardiomyocytes and myofibroblasts co-cultures stiffening in fibrotic conditions, but whether the increase in cell stiffness occurs in myofibroblasts or in cardiomyocytes or in both is yet to be determined. Michaelson and coworkers have shown that diabetic conditions lead to increased stiffness in cardiomyocytes, but not in fibroblasts [77]. My hypothesis is that during fibrosis both cell types undergo mechanical changes additionally triggered by: $(i)$ a release of chemical factors (TGF- $\beta 1$ ) from cardiomyocytes that has a direct effect on fibroblasts (myofibroblast transformation) and possibly a feedback effect on themselves; (ii) an overproduction of collagen that directly interacts with both cardiomyocytes and fibroblasts, and (iii) the contraction of mechanically coupled adjacent cardiomyocytes and myofibroblasts [78]. These three mechanisms are summarised in figure 4.2.

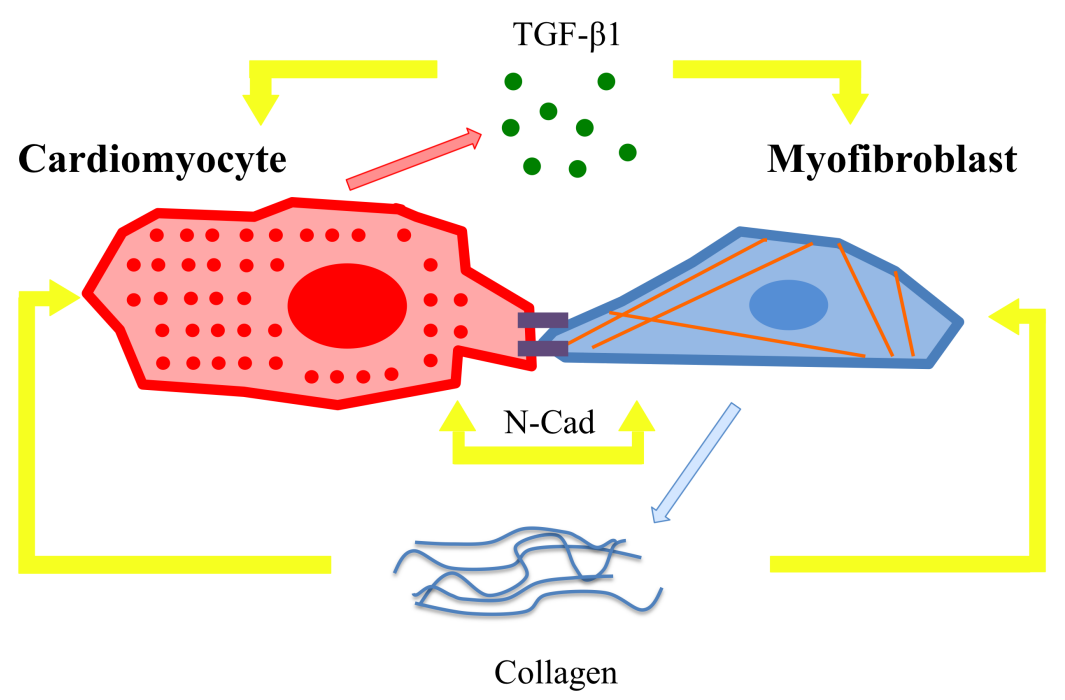

Figure 4.2: Factors influencing mechanical properties during cardiac fibrosis. 


\subsection{Ventricular Remodeling: Effect of High Shear Stress}

The early stage of post-infarction remodeling is characterised by high shear stresses exerted on cardiac laminar sheets during contraction due to the increase of interstitial space in between each cellular layer. This space is filled by a fluid that allows the diffusion of cellular nutrients and waste products and provides a specific physiological mechanical environment to cells. In post-infarction conditions, it might influence several processes at cellular level and play a critical role in fibrogenesis. In this context, with my work I studied the influence of pathological values of shear stress on cardiomyocyte monolayers by applying laminar fluid flow stimulation. The effect of increased shear stress is not completely understood especially during the whole ventricular remodeling process after the infarction

Moreover, it is important to understand the cell response, apart from biophysical and biochemical changes, to the increased shear stresses during the whole ventricular remodeling process after the infarction. I characterised the effect of high shear stresses on cardiomyocytes behaviour at short- and long-time scales. To my knowledge the physiological values of shear stress at which ventricular cardiomyocytes are subjected in vivo were never reported. Hence, I estimated it based on a model which represents cardiac laminae with parallel plates as presented for atrial cardiomyocytes by Boycott and coworkers [9]. For a beating frequency of $2.5 \mathrm{~Hz}$, the physiological ventricular shear stress in neonatal cardiomyocytes in this work turns out to be $0.17 \mathrm{dyn} / \mathrm{cm}^{2}$. Therefore, in order to simulate post-infarction conditions, shear stresses one order of magnitude higher were used to stimulate cardiomyocytes monocultures. Morphological changes of cardiomyocyte under fluid flow were monitored and quantified by using Electric CellSubstrate Impedance Sensing (ECIS) [47]. This non-optical technique allows to measure variations in electrical impedance of a cellular monolayer indicative of cell-shape and cell-cell connection changes continuously. Advantages such as non-invasiveness, good throughput and high reproducibility make this method suitable to investigate the effect of high shear stress on delicate primary neonatal cardiomyocytes.

In this work I observed that exposing cardiomyocytes to high shear stress for $2.5 \mathrm{~h}$ induced a sharp and exponential increase in the electrical impedance of the monolayer (for 30-50 min) followed by a slower linear increase (for $1.5 \mathrm{~h}$ ), while the impedance of non-sheared monolayers remained constant, as shown in figure 3.9. This indicates that pathological values of shear stress affect the morphology of cardiomyocytes, reflected by the variation of electric impedance. The possibility that this variation was partially or completely caused by the movement of ions constituting the cell medium under flow stimulation was excluded by performing a control experiment of sheared samples without cells, where the impedance decreased slightly over time (see figure 3.10). Thus, the increase of electric impedance is a clear result of active changes in the cardiomyo- 
cyte monolayer under flow exposure. By employing an optical technique, Reflection Interference contrast microscopy (RICM), I correlated the sharp exponential raise of the electric impedance with an immediate decrease in the average distance between the ventral part of the cardiomyocytes and the substrate. This result is also supported by a quantitative study of endothelial cell adhesion under flow that showed an average decrease in distance between cell membraneand substrate [79]. When stimulated at a shear rate of $4 \mathrm{dyn} / \mathrm{cm}^{2}$, the cadiomyocyte monolayers approached the surface during the first $15 \mathrm{~min}$, following which they slowly withdrew. The height of the cell-substrate space increased again and reached the same value as in unstimulated conditions within $1.5 \mathrm{~h}$. Interestingly, during this process the cell contact area remained constant, and started to slightly increase only thereafter. It seems that the decrease of cell-substrate distance and the increase of cell-substrate contact area are two consecutive temporal events. The first exponential increase in cardiomyocytes electric impedance can be correlated with the decrease in the cell-substrate distance, while the second linear increase can be explained by a further cell spreading. Similar experiments were performed by DePaola et al. on bovine aortic endothelial monolayers and they observed a peak in electrical impedance when exposed to shear stress of $10 \mathrm{dyn} / \mathrm{cm}^{2}$ at the flow onset. By comparing their observations to the theoretically predicted behaviour according to the ECIS model equation they concluded that the increase in impedance is caused by both, a decrease in the cell-substrate distance and by an increase of the cell radius [80]. My observations in section 3.2.1 partially confirm those results as sheared cardiomyocytes approach immediately the surface, causing an increase in electric impedance, without any increase of cellular dimensions. Moreover, DePaola and coworkers measured a decrease in electrical impedance 15 min after the peak at the flow onset and they related it to an adaptive response of the endothelial cells to the fluid stimulation. On the contrary, I observed a continuous increase in electrical impedance of the cardiomyocytes during the whole shear stimulation which is caused by an increase in cell-substrate contact area $1.5 \mathrm{~h}$ after the flow onset. The diverse behaviour of these two cell types under high levels of shear stress right after they approach the surface at the flow onset is probably due to the different nature of the cells. Excitable cells such as cardiomyocytes may present a different transduction and response to stimuli.

According to my results applying increasingly high shear stresses $\left(1.59 \mathrm{dyn} / \mathrm{cm}^{2}\right.$, $\left.4 \mathrm{dyn} / \mathrm{cm}^{2}, 6 \mathrm{dyn} / \mathrm{cm}^{2}\right)$ to cardiomyocytes induced the same immediate response but with different speed. A shear stress of $4 \mathrm{dyn} / \mathrm{cm}^{2}$ and $6 \mathrm{dyn} / \mathrm{cm}^{2}$ triggered a five times faster exponential increase of the cell impedance compared to $1.59 \mathrm{dyn} / \mathrm{cm}^{2}$. It seems that $4 \mathrm{dyn} / \mathrm{cm}^{2}$ is a threshold value for shear stress beyond which there is no difference in the cellular response to the flow onset. Boycott and coworkers observed no changes in magnitude of adult atrial cardiomyocytes response to shear stresses higher 
than $2.8 \mathrm{dyn} / \mathrm{cm}^{2}$ in terms of increased outward potassium current causing fast repolarization [9]. However, the biological differences (gene expression, structural proteins, and transcription factors [81]) between isolated atrial and ventricular cardiomyocytes might cause differences in reaction to increasingly values of shear stress. This would explain the different threshold value of the shear stress between their experiments and those presented in this work. Regardless which pathological shear stress the cardiomyocytes are exposed to, they approach and withdraw from the surface. However, the speed of this response is faster for higher flow stimulations. This might be related to the reorganisation of cytoskeleton in response to mechanical stimuli.

Cytoskeleton plays an important role in the cellular mechanotransduction and it may transmit shear stress signal to the nucleus via actin filaments or microtubules. Nishimura and coworkers observed high compression of microtubules induced by application of shear stress to adult cardiomyocytes [82]. Based on their results, I speculate that when high shear stress is applied to cardiomyocytes, the compressed microtubules push the cells towards the substrate and this response is faster when the strength of the stimulation increases. Also the degree and the rate of microtubule polimerization/depolimerization might be affected by the high flow stimulation. Indeed, it has been reported that in hypertrophic conditions, typical for post-infarction remodeling, cardiomyocytes are characterised by an elevated level of microtubules polymerization [83].

In my work I observed only cellular morphological changes at the shear stress onset: cardiomyocytes beating frequency, extracted from the periodic oscillation of electric impedance, does not seem to be affected by the sudden exposure of the cells to flow. Cardiomyocytes contractility was not affected by any applied shear stress value $\left(1.59 \mathrm{dyn} / \mathrm{cm}^{2}\right.$, $\left.4 \mathrm{dyn} / \mathrm{cm}^{2}, 6 \mathrm{dyn} / \mathrm{cm}^{2}\right)$. On the contrary, exposing cardiomyocytes monolayers to laminar flow for $108 \mathrm{~h}$ induced significant changes on cardiomyocytes morphology, connectivity and contractility. The electrical impedance of cardiomyocytes continuously increased until the flow stimulation was stopped as shown in figure 3.14. When cells were exposed to $1.59 \mathrm{dyn} / \mathrm{cm}^{2}$ and $4 \mathrm{dyn} / \mathrm{cm}^{2}$ shear stress the final increment in impedance was two and three times higher than the value before the flow onset, respectively. There was no further increment when a shear stress higher than $4 \mathrm{dyn} / \mathrm{cm}^{2}$ was applied to cardiomyocytes, pointing again to the fact that $4 \mathrm{dyn} / \mathrm{cm}^{2}$ is a threshold value for the cell response. I correlated the first $20 \mathrm{~h}$ of impedance increment with an increase of cellsubstrate contact area, that started $1.5 \mathrm{~h}$ after the flow onset. Cardiomyocytes further spread under high flow stimulation, reduce intercellular distances, which results in an increase of the electric impedance. They form larger focal adhesions, however maintaining their average cell-substrate distance. A similar result was observed for 3D fibroblast cultures stimulated with low shear stress. After $48 \mathrm{~h}$ of stimulation fibroblasts spreading increased in the presence of TGF- $\beta 1$ [84]. During post-infartion remodeling it is 
well know that cardiomyocytes start to secrete paracrine mediators like which TGF- $\beta 1$. Probably high shear stress levels might be one of the physical trigger for the secretion of TGF- $\beta 1$ that can mediate the further spreading observed in this work, similar to the work of others [84]. Further analysis concerning TGF- $\beta 1$ effect on cardiomyocytes under shear stress could lead to a confirmation of this hypothesis.

It should be noted that in this work I measured the cardiomyocyte-substrate contact area and not the complete cell size. However, high values of shear stress are typical in cardiac hypertrophic conditions and so the possibility that cardiomyocytes increase their volume should not be excluded. Indeed, Hariharan and coworkers demonstrated that high shear flow induces an increase in ERK phosphorylation in cardiomyocytes, involved in hypertrophic response [12].

The continuous increase in cardiomyocytes electric impedance under pathological values of shear stress was caused also by changes in cell-cell connections and cell membrane composition, unfolding or thickness. By using the ECIS area contact model introduced by Lo et al. [85] it was possible to correlate impedance increase with the variation in the barrier resistance $R_{b}$ describing the resistance to current flow in the intercellular gap and therefore, the density of cell-cell contact, the parameter characterising cell-substrate distance $\alpha$, and the membrane capacitance $C_{m}$. Already after $48 \mathrm{~h}$ of flow stimulation with a shear stress of $1.59 \mathrm{dyn} / \mathrm{cm}^{2}$ I observed an increase in resistance barrier $R_{b}$ describing the tightness of cell-cell contacts. $R_{b}$ increased by a factor of 1.5 compared to the non-sheared cardiomyocytes. Analogously cell-cell contact increased after $108 \mathrm{~h}$ of shear stimulation indicating a direct proportionality between $R_{b}$ and the flow exposure time. The enhanced connectivity in presence of high shear stress is probably due to an increased expression of both gap and adherens junctions (Cx43 and N-Cad) by the cardiomyocytes. Two slightly different studies confirm the high level of these two connection proteins in sheared ventricular cardiomyocytes $[12,86]$. Hariharan et al reported an increased amount of $\mathrm{N}-\mathrm{Cad}$ and $\mathrm{Cx} 43$ in response to $4 \mathrm{~h}$ oscillatory shear stress $\left(6 \mathrm{dyn} / \mathrm{cm}^{2}\right)$ and no significant change in cell-cell adhesion strength [12]. Dvir and coworkers observed activation of ERK1/2 signaling cascade, inducing synthesis of cell-cell contact proteins, upon low pulsatile fluid flow stimulation $\left(0.6 \mathrm{dyn} / \mathrm{cm}^{2}\right)$ for $24 \mathrm{~h}$ and increased $\mathrm{N}-\mathrm{Cad}$ and $\mathrm{Cx} 43$ expression [86]. In both studies they found an increased level of cell connections earlier than $48 \mathrm{~h}$ as I measured. Although, this discussion lacks biochemical analysis, I can not exclude that also in my experiments the signal cascade and the overexpression of the gap and adherens junction proteins started before $48 \mathrm{~h}$. Based on my observations I assume that cardiomyocytes start to further spread within a time window of $20 \mathrm{~h}$ to $40 \mathrm{~h}$, and then increase the amount of cell-cell junctions. Moreover, the different type of flow stimulation that Hariharan, Dvir and coworkers used (oscillatory and pulsatile flow, respectively) compared to the one employed in this 
work (continuous laminar flow) could have influenced the time response of cardiomyocytes to the shear stress, resulting in an early increase of $\mathrm{N}$-Cad and Cx43 expression. Summing up, it is clear that shear stress triggers the overexpression of electrical and adhesion proteins that leads to an enhanced connectivity between cardiomyocytes. The way the cells are stimulated might influence the time scale of their reaction to the flow.

As described above the optical measurements showed that the cell-substrate distance decreased at the flow onset and afterwards it remained almost constant. According to the impedance spectroscopy measuraments, $\alpha$ in the cardiomyocytes exposed to shear stress of $1.59 \mathrm{dyn} / \mathrm{cm}^{2}$ for $48 \mathrm{~h}$ and $108 \mathrm{~h}$ is 3.5 times higher than the non-sheared culture. $\alpha$ is inversely proportional to the square root of the cell-substrate distance $(h)$, directly proportional to the cell width $W$, and the square root of the resistivity of the bulk cell medium $\rho\left(\alpha=(1 / 2) W(\rho / h)^{\frac{1}{2}}\right)$. Assuming that the electrical properties of the cell medium do not change ( $\rho$ is constant), the increase in cardiomyocytes cell-substrate contact area, observed optically in the first 20-40 h of flow stimulation, can be responsible for the rise of $\alpha$. I observed that $\alpha$ further increases up to $48 \mathrm{~h}$ of flow stimulation, which I relate to the increase in the cell-substrate contact area with the cell-substrate distance remaining constant. After this time point, $\alpha$ remained constant, which infers that the cell-substrate distance and cell-substrate contact area do not change until $108 \mathrm{~h}$ of flow stimulation.

One should also take the membrane capacitance $C_{m}$ into account in order to explain the increase in the electrical impedance of the cardiomyocytes exposed to shear stress of $1.59 \mathrm{dyn} / \mathrm{cm}^{2}$. The value of $C_{m}$ decreased by 4 and 2.5 times when the shear stress was applied for $48 \mathrm{~h}$ and $108 \mathrm{~h}$, respectively. Sato et al also reported an increase in mechanical stiffness of endothelial cell membranes exposed to shear stress [87]. This may suggest that under flow stimulation the cell membrane became less rough, decreasing the total membrane area. The change in the mechanical properties, permeability [80] or its membrane composition, for example expression of more or new adhesion molecules or membrane proteins, will have an effect on the value of $C_{m}$. Such rearrangements in the membrane may have occurred in cardiomyocytes during my experiments that led to a decrease of $C_{m}$. Indeed I do optically observe an increment of the size of focal adhesions after $48 \mathrm{~h}$ of flow stimulation. It has also been demonstrated that shear stress alters the flux of ions through the cardiomyocyte membrane: shear of $0.5 \mathrm{dyn} / \mathrm{cm}^{2}$ induces an increase in outward current of atrial cardiomyocytes [9]. This may also occur in ventricular cardiomyocytes under shear stress and induce changes in $C_{m}$.

After $108 \mathrm{~h}$ of flow stimulation, $R_{b}$ and $C_{m}$ were observed to vary as function of the applied shear stress (figure 3.15). Indeed $R_{b}$ increased and $C_{m}$ decreased for increasing values of shear stress $\left(1.59 \mathrm{dyn} / \mathrm{cm}^{2}, 4 \mathrm{dyn} / \mathrm{cm}^{2}, 6 \mathrm{dyn} / \mathrm{cm}^{2}\right)$. This supports the idea that the shear stimulation enhance the cell-cell connectivity and the protein composi- 
tion in membranes. Furthermore, trends show that higher shear stresses amplify this response, for example $R_{b}$ of cells sheared at $6 \mathrm{dyn} / \mathrm{cm}^{2}$ was three times higher than $R_{b}$ of cell sheared to $1.59 \mathrm{dyn} / \mathrm{cm}^{2}(\mathrm{P}<0.001)$. Interestingly, there is no difference in $R_{b}$ or $C_{m}$ increasing the shear stress values exposing cardiomycytes to only $48 \mathrm{~h}$ of stimulation. Probably in cardiomyocyes exposed to flow some mechanisms, sensitive to the increase of shear stresses, are activated only after $48 \mathrm{~h}$. I believe that these mechanisms are connected to the flow-mediated enhance in cell connectivity, supporting the above mentioned hypothesis that cardiomyocytes start to form adherens and gap junctions about $48 \mathrm{~h}$ after the flow onset.

The increase in cardiomyocytes connectivity and changes in cellular membrane properties under high shear stress are not the only factors that cause the rise in electric impedance over time. The force applied by the flow promotes a reorganisation of the cytoskeleton of the cell in such a way that the actin fibers are reoriented in the direction of the flow. I used a custom written program to quantify the net orientation distribution via two parameters: $e$, eccentricity of an ellipse that fits the normalised fiber orientation distribution in an image; and $\theta$, the angle along which the largest number of fibers is oriented. I observed that when a shear stress of $4 \mathrm{dyn} / \mathrm{cm}^{2}$ was applied for $108 \mathrm{~h}$ to cardiomyocytes the average $\langle e\rangle$ of the distribution was equal to 0.97 and $\theta$ equal to $-9.7^{\circ}$. Referring back to section 2.4.1.3, this means that at least $2 / 3$ of the actin fibers were oriented parallel to the direction of the flow $\left(\theta=0^{\circ}\right)$. On the other hand, non-sheared cardiomyocytes were randomly oriented $(<e>=0.62)$. Moreover, analysing the orientation distribution of the actin fibers as function of the duration of shear exposure revealed that the percentage of these fibers oriented along the flow direction increased. It required $108 \mathrm{~h}$ of shear stimulation to reorganize $70 \%$ of the fibers along the flow direction, which represents the response of the cytoskeleton to the shear stress. To my knowledge the reorientation of the actin fibers of cardiomyocytes under fluid flow stimulation in 2D has not been reported until date. However, it has been observed for other cell types by several groups [88-90]. Van der Meer et al reported a reorganisation of actin fibers due to the presence of shear stress in endothelial cells exposed for $10 \mathrm{~h}$ and $24 \mathrm{~h}$ to $15 \mathrm{dyn} / \mathrm{cm}^{2}$ [88]. Another study demonstrated that focal adhesions formation is crucial in regulating endothelial cell response to laminar flow [89]. Archambault and coworkes showed that rabbit tendon fibroblasts tended to align along the direction of the flow at $25 \mathrm{dyn} / \mathrm{cm}^{2}$ stimulation for $3 \mathrm{~h}$ [90]. They observed that the mechanotransduction of the shear stress does not depend on calcium concentration and that other pathways may be involved. Actin fiber reorientation in cardiomyocytes as response to shear stress may be triggered by the activation or modification of integrins. These transmembrane proteins are involved in ECM adhesion and in translation of external mechanical stimuli into biochemical signals. My hypothesis is that the ap- 
plication of shear stress may induce orientation of the fibers in the ECM, on which cardiomyocytes adhere, in the direction of the flow in order to reduce the fluid drag through the ECM grid. This provides contact guidance, mediated via integrins, for the remodeling and alignment of the actin fibers in cardiomyocytes. Indeed, several studies have demonstrated how cardiomyocyes remodel their shape and myofibrils according to geometrical patterns $[91,92]$. Ng Ping and coworkers have shown the important role of $\alpha_{1} \beta_{1}$-integrin in the alignment of the ECM matrix and fibroblasts in a $3 \mathrm{D}$ system under low shear stress [84]. In my experiments, integrins may play an important role in the spatiotemporal organisation of the actin fibers and also in the reorientation of the cardiomyocytes along the direction of the flow.

Overall, I successfully demonstrated that pathological values of shear stress induce a reorientation of actin fibers and cardiomyocytes in the direction of the flow. This anisotropy is time dependent, requires more than $72 \mathrm{~h}$ of stimulation, and is mediated via contact guidance. These cell morphological changes together with the enhanced cellcell connectivity cause the continuous increase in electric impedance of cardiomyocytes under flow stimulation.

A long-term exposure of cardiomyocytes to high shear stress has an effect on the contraction frequency. Compared with the non-sheared co-cultures, it increased with the application of fluid flow and also with the magnitude of the shear stress $\left(1.59 \mathrm{dyn} / \mathrm{cm}^{2}\right.$ $4 \mathrm{dyn} / \mathrm{cm}^{2}$ and $6 \mathrm{dyn} / \mathrm{cm}^{2}$ ). These results were confirmed by Lorenzen-Schmidt and coworkers that observed an increase in beating rate with the shear [11]. In addition, I observed that there is no significant difference between the beating frequency of cardiomyocytes sheared at $4 \mathrm{dyn} / \mathrm{cm}^{2}$ and $6 \mathrm{dyn} / \mathrm{cm}^{2}$. Another proof that $4 \mathrm{dyn} / \mathrm{cm}^{2}$ is a threshold value of shear stress beyond which there is no difference in the cellular response in terms of contractility. Interestingly, at any applied shear stress the increase in beating frequency was not constant over time but reached a maximum after $48 \mathrm{~h}$ of shear stress. Cardiomyocytes sheared with $4 \mathrm{dyn} / \mathrm{cm}^{2}$ and $6 \mathrm{dyn} / \mathrm{cm}^{2}$ for $108 \mathrm{~h}$ were beating even slower than the non-sheared samples. I speculate that myofibroblasts residual from cardiomyocytes isolation may be responsible for the decrease in beating frequency after $48 \mathrm{~h}$ of shear stimulation. It has been reported by $\mathrm{Ng}$ Ping that low levels of interstitial flow induce myofibroblast proliferation. It is also possible that myofibroblasts increase heterocellular and homocellular connections similarly to the flow stimulated cardiomyocytes, as already presented in this work. This induces changes in the electromechanical coupling, as presented for fibrotic co-culture with increasing ratio of myofibroblasts, and leads to a decrease in the beating frequency of the cardiomyocytes. On the other hand, one potential cause of the increase of beating frequency before $48 \mathrm{~h}$ of stimulation could be related to the above mentioned activation of ERK1/2 under shear stress. Dvir and coworkers reported that pulsatile fluid flow activated ERK $1 / 2$ inducing synthesis of 
contractile proteins $\alpha \mathrm{SAn}$ and Tn-T [86]. They measured high levels of these proteins expression after $24 \mathrm{~h}$ of shear stress stimulation. Flow-induced enhancement of contractile protein expression could lead to higher beating frequency. Lorenzen-Schmidt et al also observed an increase in contraction frequency in cardiomyocytes and showed that stretch-activated channels are probably not involved in the process [11]. It is known that the $\beta$-adrenergic signalling pathway has an influence on the beating rate by regulating the influx of $\left[\mathrm{Ca}^{2+}\right]$ through the L-type $\left[\mathrm{Ca}^{2+}\right]$ channel. Lorenzen-Schmidt et al. suggest that shear stress can induce an increase in $\left[\mathrm{Ca}^{2+}\right]$ current influx and activate a signalling pathway similarly to that of the $\beta$-adrenergic one. Integrins may also play an important role in triggering actin fibers and cell orientation along the flow direction as well as in increasing cardiomyocyte contractility. $\beta 1$ integrins are linked to L-type $\mathrm{Ca}^{2+}$ channels [93]. Therefore, it is reasonable to assume that growth factors that bind to $\beta 1$ integrins [11], and flow-induced changes in ECM, as I described above, may increase the beating frequency in cardiomyocytes. The mechanisms that may influence the increase of contractility in cardiomyocytes under shear stress are summarised in the figure 4.3.

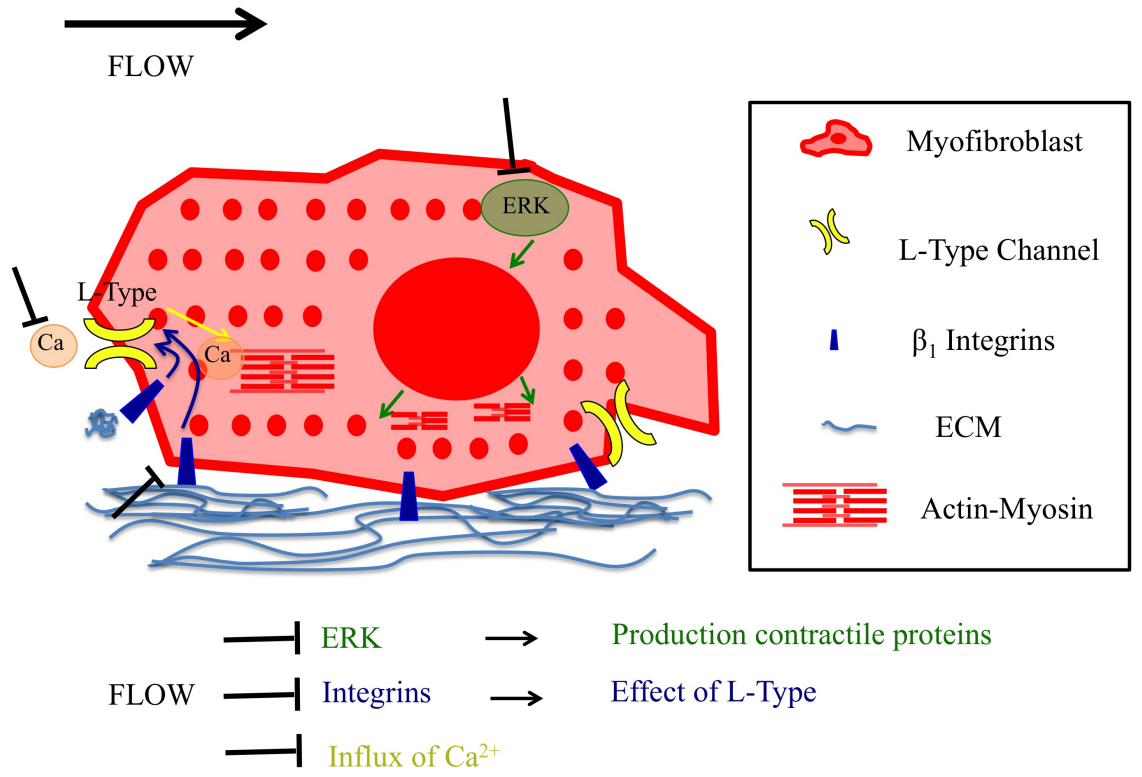

Figure 4.3: Mechanisms that may induce an increase in contractility of cardiomyocytes under shear stress.

Another flow-induced effect of shear stress on cardiomycytes contraction is the synchronisation of the beating frequency. Cells in different part of the sample coordinated their activity when sheared (beating frequency $3 \pm 0.05 \mathrm{~Hz}$ ), while non-stimulated cardiomyocytes were characterised by different beating frequencies $(1.6 \pm 0.53 \mathrm{~Hz})$. Probably the enhanced cell-cell connectivity due to the presence of the flow enables cells to syn- 
chronise the beating. After observing the fact that the cardiomyocytes contractility is sensitive to shear stress, I tested the effect of flow stimulation on beating frequency also in co-cultures of fibroblasts and cardiomyocytes. Co-cultures with $50 \%$ and $25 \%$ fibroblasts under $6 \mathrm{dyn} / \mathrm{cm}^{2}$ shear stress stimulation for $48 \mathrm{~h}$ were beating 1.6 and 2 times faster than the non-sheared cell, respectively. A better understanding of this response can be reached by analysing the effect of shear stress on each cell type individually. As mentioned above, this response in cardiomyocytes may be caused by the $\beta$-adrenergic signalling pathway, an increase in $\left[\mathrm{Ca}^{2+}\right]$ current, integrins activation, and an increase in contraction proteins. This may be the main reason why sheared co-cultures beat faster than non-sheared ones. Shear stress on fibroblasts induces the expression of $\alpha$-SMA and the transformation into myofibroblasts, as reported by $\mathrm{Ng}$ Ping and coworkers [84]. In their study, they observed that after 5 days of flow stimulation over $95 \%$ of the fibroblasts were myofibroblasts. However, the shear stress they applied was one order of magnitude lower than the one I used to stimulate the co-cultures in my experiments. I speculate that higher fluid stress may accelerate the flow-induced transformation of fibroblasts into myofibroblasts, and induce contraction after 2 days. I also believe that shear stress leads to an increase of junctional protein expression in myofibroblasts as it does in cardiomyoctes. The flow-induced increase in beating frequency in cardiomyocytes is damped by the large amount of myofibroblast-cardiomyocyte connections. Nevertheless, the beating frequency in sheared is higher than non-sheared co-cultures, and obviously lower than cardiomyocyte monocultures.

In this work, I observed that co-cultures with $90 \%$ myofibroblasts after $48 \mathrm{~h}$ of shear stimulation were beating, whereas no activity was recorded for non-sheared co-cultures. This is the first time such a flow-induced contractility in a fibrotic co-culture with 9:1 ratio of myofibroblasts and cardiomyocytes was observed. My hypothesis is that the fluid flow-enhanced connectivity in myofibroblasts enables propagation of electrical signals via Cx43 over large distances. The increased expression of gap junctions may overcome the previously known spatial limit of $300 \mu \mathrm{m}$ for impulse propagation between two cardiomyocytes through myofibroblasts [66]. This mechanism is probably used in vivo during fibrosis to propagate the electric signal through the scar. This suggests that the increase of shear stress during post-infarction is involved in the myofibroblast transformation and may facilitate the electrical conduction in the infarcted area, playing an important role in fibrogenesis.

To summarise, I have shown that high values of shear stress, which mimics the early stage left ventricular remodeling, induce morphological and functional changes in cardiomyocytes at different time scales. The main results of the presented discussion can be noted as follows:

1. An immediate decrease of the cell-substrate distance occurs at the flow onset. 
This response, probably mediated by microtubules, varies with the magnitude of the shear stress.

2. After two hours cell-substrate contact area starts to increase and probably the activation of signaling pathways induces synthesis of high levels of contractile and cell-cell contact proteins.

3. At $48 \mathrm{~h}$ of flow stimulation, the cell beating frequency reaches a maximum.

4. Between 48 and $72 \mathrm{~h}$ of shear stress application, cardiomyocyte actin fibers are reorganised probably via integrin-mediated contact guidance.

5. After $108 \mathrm{~h}$ of shear stress exposure, actin fibers and most likely also cardiomyocytes are oriented along the flow axes. Additionally, the anisotropic monolayer is characterised by an elevated cell-cell connection that induces a decrease in beating frequency and possibly constitute an arrhythmogenic mechanism, often observed left ventricular remodeling post-infarction.

A summarising scheme of these processes is show in figure 4.4 .

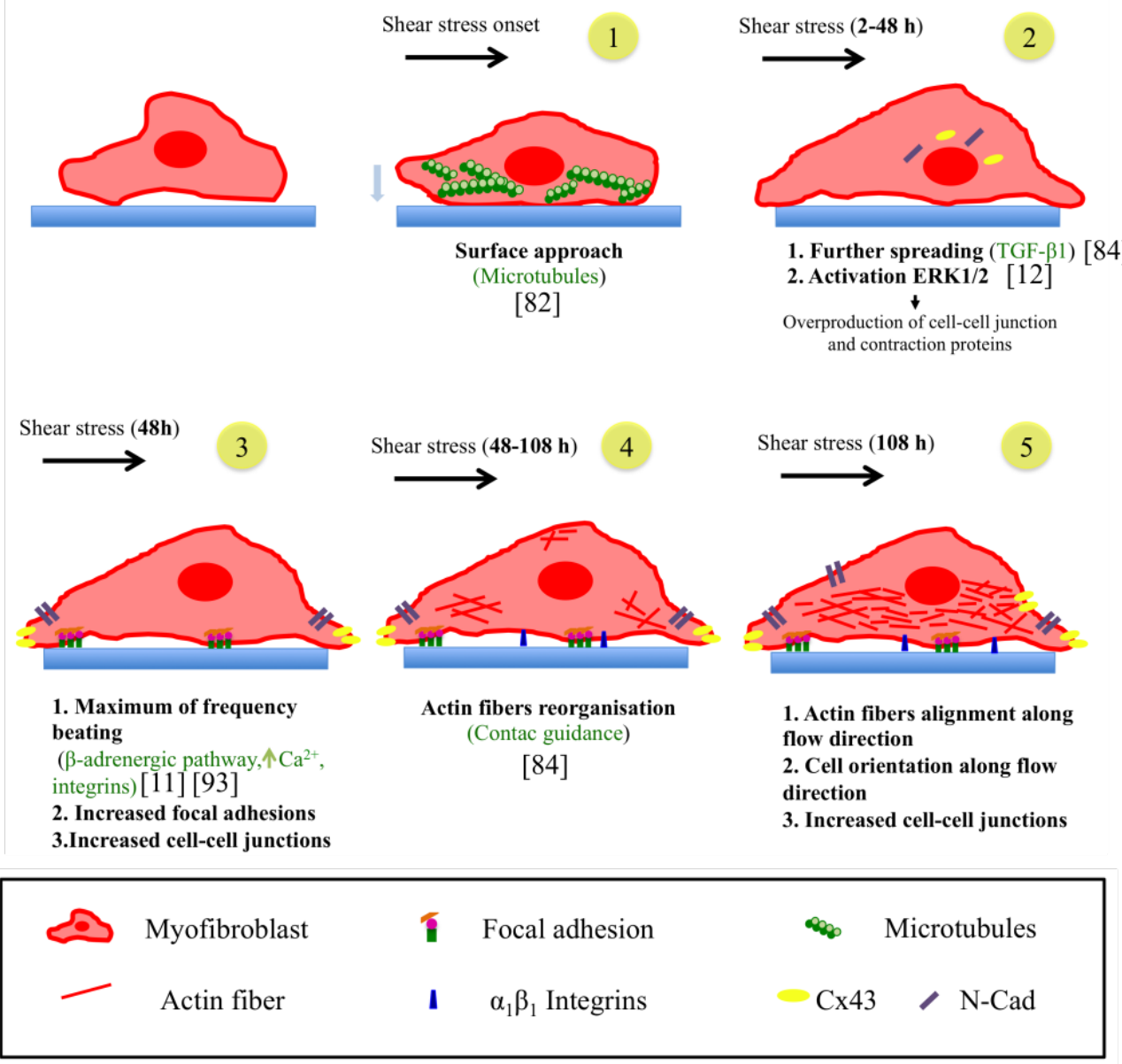

Figure 4.4: Time sequence of the effects of high shear stress on cardiomyocytes. 


\section{Conclusions and Outlook}

In this thesis, I presented a study on the influence of biophysical changes on cellular mechanical properties, morphology, and connectivity in left ventricular remodeling after myocardial infarction (MI). In particular, I focused on the effect high levels of shear stress and fibrosis, which occur in the early and late phase of ventricular remodeling, respectively.

\section{Fibrosis: connectivity and mechanics}

I was interested in how the connectivity between cells in fibrotic conditions could influence cardiac functionality. By using co-culture of cardiomyocytes and myofibroblasts as an in vitro fibrotic model, I demonstrated that their communication occurs via both electrical and mechanical junctions. Increasingly severe fibrosis (co-cultures with decreasing density of cardiomyocytes) is characterised by a linear overexpression of electrical junction protein $(\mathrm{Cx} 43)$ in myofibroblasts. An enriched Cx43 expression leads to higher possibility of cardiomyocytes-myofibroblast coupling. This induces alterations in cardiomyocytes electrophysiology: decrease in beating frequency and outbreaking of spiral waves. Several studies confirmed these findings and also reported i)changes in duration of cardiomyoctyes action potential, ii) slow conduction velocity, and iii) arrhythmogenesis due to cardiomyocytes-myofibroblasts electrical coupling [63-65]. Additionally, I observed high expression of mechanical junctions protein (N-Cad) between myofibroblasts in co-culture with low cardiomyocytes ratios. Accordingly, I proposed that myofibroblasts may influence the function of cardiomyocytes by applying contractile forces via mechanical junctions. Moreover, N-Cad might be responsible for the transmission of the contractile forces between myofibroblasts through the fibrotic scar in order to overcome the spatial limit $(300 \mu \mathrm{m}$ [66]) of action potential propagation via Cx43. These results reveal that the cardiac scar is not a passive tissue wherein the cardiomyocytes and myofibroblasts couple electromechanically. Emerging therapeutic techniques try to use this coupling to develop a "better scar" that reduces the disturbance in conduction of action potential and reduces the outbreak in arrhythmias. Since an increase in electrical junction in fibrotic model leads to alterations in electrophysiology of cardi- 
omyocytes, inhibiting Cx43 expression may represent a promising strategy. Peptides that alter $\mathrm{Cx} 43$ functions have been observed to decrease the size of the infarcted area and arrhythmogenesis [62]. With my work I also point out to the possibility of developing a new therapeutic approach by targeting the expression of N-Cad in myofibroblast as it may be beneficial in the prevention of fibrosis and all its pathological consequences.

To complete the characterisation of fibrosis, I compared the viscoelastical properties of cardiomyocytes, fibroblasts monocultures with fibrotic co-culture by employing AFMbased microrheology. Cardiomyocytes were observed to be quite stiff and exhibiting a solid-like behaviour, attributable to the high amount of contraction proteins, peculiar for this cell type. The frequency dependent viscoelastic properties were described with a slightly high power law coefficient, which indicates the agitation in their cytoskeletal network due to the copious active processes involved in cardiomyocytes beating. Fibroblasts were observed to be soft, less viscous, and their mechanical properties obey a weak power-law reflecting an increase in their cytoskeletal tension. I correlate these properties with the ability of fibroblasts to highly deform and pass through tissues, migrating towards the infarct area. Fibrotic co-culture, obtained by mixing cardiomyocytes and myofibroblasts, were stiffer than both cell types and show a solid-like behaviour at almost all frequencies. This is congruent with the typical increase in tissue stiffness that characterises fibrosis and is due to the overproduction of collagen necessary to sustain the increase in ventricular wall stresses. Interestingly, the co-culture exhibited properties of both cardiomyocytes and fibroblasts: viscosity and power law coefficient, respectively. I proposed three factors that may be responsible for changes in mechanical properties of the fibrotic co-culture: (i) TGF- $\beta$, which once is secreted by cardiomyocytes can have an effect on both cell types; (ii) overproduced collagen, that increases the stiffness of the extracellular environment, inducing stiffening in the cells; (iii) mechanical junctions, by which traction forces are propagated from cell to cell triggering viscoelastic response. In this work I measured the overall mechanical properties of the fibrotic co-culture. Whether the increase of cell stiffness occurs in fibroblasts or in cardiomyocytes or in both is yet to be determinated. Combining AFM-based microrheology with fluorescence microscopy is possible to visualise one of the two cell types and distinguish between cardiomyocytes or myofibroblasts properties in fibrotic conditions. To my knowledge this is the first time that viscoelastic properties of fibrosis condition are presented. In the recent years, most studies on mechanical properties of fibrosis were focused on the assessment of the elastic modulus of the tissue, completely neglecting the viscous properties that characterise cells. Simulations on changes in viscoelastic properties show that the infarcted tissue contribute to cardiac rhythm disturbance [76]. Therefore, a better understanding of the mechanical properties of fibrotic tissue and the mechanisms underlying their changes may lead to the identification of more efficient 
and new therapies.

\section{High shear stress on cardiac laminar sheets: a pathological model}

In this work I presented a detailed description of the effects of high shear stress applied via fluid flow to cardiac laminar sheets at different time scales. The values of applied shear stress were chosen one order of magnitude higher than a physiological shear stress estimated with Couette flow model to simulate pathological condition (high shear stress) that characterises early stage of left ventricular remodeling. By combining impedance spectroscopy and optical microscopy I could provide an overview of morphological and contractile changes of cardiomyocytes under the effect of shear stress for 4.5 days. At the shear onset cardiomyocytes approach the surface with a dynamics that accelerates with higher applied shear stress. An increase of cell-substrate contact area, cell-cell connectivity, and beating frequency occurs between 2 hours and 48 hours of flow exposure, followed by a gradual reorientation of actin fibers along the direction of the flow stimulation during the next 24 hours. Finally, after 108 hours of high shear stress stimulation I observed an increased cellular connectivity, changes in cellular membrane properties, and oriented actin fibers along the direction of flow. These cellular characteristics are the typical symptom of post-infarct or cardiopathology leading to fibrosis. Therefore, I proved the cause-effect relationship between the increased shear stress that precede the ventricular remodeling and the clinical characteristics found after MI. This indicates that stimulating cardiomyocytes with high shear stress is a reliable in vitro pathological model that can be used to test new drugs to inhibit or reverse MI effects. It is clear that elevated shear stress affects cardiomyocytes morphology and contractility. Therefore, it is necessary that the numerical simulation focusing on the prediction of the consequences of MI, cardiac pathology, and fibrogenesis take into account also the presence of high shear stress. Shear stress is often neglected in both experimental and computational simulations of post MI conditions, leading perhaps to incorrect assumptions or conclusions. Another proof of the necessity to include shear stress in in silico and in vitro models comes from one surprising result of this work: co-cultures with only $10 \%$ of cardiomyocytes are able to contract under flow stimulation, unlike in static conditions. A similar result was observed in vivo, where action potential propagated through fibrotic tissue in which cardiomyocytes were chemically removed [94]. My hypothesis is that fluid flow enhanced connectivity in cells enables electrical activity propagation over large distances, overcoming the spatial limit of $300 \mu \mathrm{m}$ for impulse propagation in vitro. Applying high shear stress in vitro I was able to recreate certain conditions that are only observed in vivo, confirming the importance of this type of stimulation. 
A detailed biochemical confirmation can be carried out in the future to confirm the proper electro-mechanical coupling of cardiomyocites under shear stress. Optical mapping of action potential waveforms within the sheared monolayers might show whether the electrical conduction is altered by the flow.

In order to optically verify the enhanced expression of mechanical and electrical junctions in cardiomyocyes exposed to shear stress, immunostaining of connection proteins such as Cx43 and N-Cad should be performed. By employing 3D confocal microscopy one can verify whether in an in vitro system sheared cardiomyocytes increase their volume as it happens in an in vivo hypertrophic tissue. Other interesting experiments can be performed to study of the effect of different shear stress values on the dynamics of actin fibers reorientation, microtubules polymerisation and the analysis of the response of cardiomyocytes to an oscillating flow stimulation that simulates more realistic conditions (in vivo shear stresses are applied at each contraction). Collagen in sheared samples with and without cells could be optically investigated by fluorescence microscopy in order to confirm the involvement of shear stress in the reorientation of ECM fibers. Also, the analysis of possible changes in viscoelastic properties of cardiomyocytes exposed to shear stress may be carried out by performing microrheological experiments. Finally, the increase in the amount of focal adhesions should be correlated with the presence of fluorescently stained integrins, and study their dependence on the concentration of TGF- $\beta 1$ in the medium. To confirm that ECM fibers reorient due to the shear stress, collagen in sheared samples with and without cells should be optically visualised with fluorescence microscopy. It would be worth to analyse possible changes in viscoelastic properties of cardiomyocytes exposed to shear stress performing microrheological experiments.

To summarise, in this work I presented how changes in cellular mechanical properties and cellular connectivity can explain the disturbed electro-mechanical coupling in cardiac fibrosis. These findings might be extremely relevant to develop new therapies, especially with the idea of engineering a "better scar". I presented also how a biophysical change in the microenvironment of post MI (high shear stress) might explain the appearance of morphological and functional properties characteristic for left ventricular remodeling. 


\section{List of Figures}

1.1 The laminar sheet architecture of ventricular myocardium . . . . . . . 5

1.2 Cardiac muscle morphology . . . . . . . . . . . . . . . 7

1.3 Sarcomere structure . . . . . . . . . . . . . . . . . . . . . . 9

1.4 Cardiomyocyte Excitation-Contraction Coupling . . . . . . . . . . . . . . 12

1.5 Schematic view of electromechanical integration . . . . . . . . . . . . 13

1.6 Schematic representation of pathophysiology of ventricular remodeling . . 14

2.1 Substrates and the respective seeded cardiomyocytes/fibroblasts ratio . . 20

2.2 Scheme of myocardial sheets for shear stress calculation . . . . . . . . . . 23

2.3 Perfusion set up . . . . . . . . . . . . . . . . . . . . . . . . 24

2.4 Working principles of ECIS . . . . . . . . . . . . . . 27

2.5 Algorithm for cell connectivity quantification . . . . . . . . . . . . . 33

2.6 Fiber orientation image analysis . . . . . . . . . . . . . . . . 35

2.7 Butterworth band-pass filter . . . . . . . . . . . . . . . 36

$2.8 \theta_{\text {err }}$ as function of eccentricity . . . . . . . . . . . . . . . . . 37

2.9 Schematic drawing of AFM principles . . . . . . . . . . . . . . 38

2.10 Schematic drawing of the ATM-based microrheology . . . . . . . . . . . . 39

2.11 Schematic drawing of the hydrodynamic drag correction . . . . . . . . . 41

3.1 Electrical junctions . . . . . . . . . . . . . . . . . . . . 47

3.2 Mechanical junctions . . . . . . . . . . . . . . . . . . . . . . . . . . . 49

3.3 ECIS quantification of fibrotic connectivity . . . . . . . . . . . . 50

3.4 Beating coupling quantification via ECIS . . . . . . . . . . . . . 51

3.5 Myofibroblasts transformation . . . . . . . . . . . . . . . . . 52

3.6 Functional coupling between cardiomyocytes and fibroblasts via phase contrast imaging . . . . . . . . . . . . . . . . . 52

3.7 Mechanics of cardiomyocytes and fibroblasts monocultures and co-cultures 54

3.8 Frequency dependency of loss tangent . . . . . . . . . . . . . . 55

3.9 Impedance of sheared and non-sheared cardiomyocytes at the flow onset . 56

3.10 Impedance of uncoated and coated samples under shear stress . . . . . . 57 
3.11 RICM intensity at the flow onset . . . . . . . . . . . 58

3.12 Cell-substrate contact area under short- term shear stress exposure . . . 59

3.13 Cardiomyocytes beating frequency at the flow onset . . . . . . . . 60

3.14 Impedance of cardiomyocytes under long-term exposure to different shear stresses . . . . . . . . . . . . . . . . . . . . 6 61

3.15 ECIS model parameters for cardiomyocytes non-exposed and exposed to different shear stresses . . . . . . . . . . . . . . . 62

3.16 RICM intensity in the first $19 \mathrm{~h}$ of flow stimulation . . . . . . . . 63

3.17 Effects of $19 \mathrm{~h}$ for shear stress on cardiomyocytes impedance and cellsubstrate contact area . . . . . . . . . . . . . . . 64

3.18 Orientation distribution of fibers with and without flow . . . . . . 65

3.19 Fibers orientation over time under shear stress . . . . . . . . . . . . 67

3.20 Time course of the beating frequency of cardiomyocytes exposed to different shear stresses . . . . . . . . . . . . . . . . . 68

3.21 Cardiomyocytes beating frequency after $48 \mathrm{~h}$ of stimulation at different shear stresses . . . . . . . . . . . . . . . . . . . 69 69

3.22 Beating frequency of co-cultures under shear stress . . . . . . . . . 70

4.1 Mechanoelectrical crosstalk in in cardiac fibrosis . . . . . . . . . . . . 76

4.2 Factors influencing mechanical properties during cardiac fibrosis . . . . . 79

4.3 Mechanisms that may induce contractility increase . . . . . . . . . . 87

4.4 Time sequence of the effects of high shear stress . . . . . . . . . . . 89 


\section{Bibliography}

[1] A. Galli and F. Lombardi. "Postinfarct Left Ventricular Remodelling: A Prevailing Cause of Heart Failure". Cardiology research and practice 2016 (2016).

[2] E. L. Ongstad and R. G. Gourdie. "Can heart function lost to disease be regenerated by therapeutic targeting of cardiac scar tissue?" In: Seminars in cell \& $\mathcal{S}$ developmental biology. Vol. 58. Elsevier. 2016, pp. 41-54.

[3] S. Paul. "Ventricular remodeling". Critical care nursing clinics of North America 15.4 (2003), pp. $407-411$.

[4] J. Zhuang, K. A. Yamada, J. E. Saffitz and A. G. Kléber. "Pulsatile stretch remodels cell-to-cell communication in cultured myocytes". Circulation research 87.4 (2000), pp. 316-322.

[5] Y. Zhang, R. B. Sekar, A. D. McCulloch and L. Tung. "Cell cultures as models of cardiac mechanoelectric feedback". Progress in biophysics and molecular biology 97.2 (2008), pp. 367382.

[6] K.-G. Shyu, C.-C. Chen, B.-W. Wang and P. Kuan. "Angiotensin II receptor antagonist blocks the expression of connexin 43 induced by cyclical mechanical stretch in cultured neonatal rat cardiac myocytes". Journal of molecular and cellular cardiology 33.4 (2001), pp. 691-698.

[7] K. Yamada, K. G. Green, A. M. Samarel and J. E. Saffitz. "Distinct pathways regulate expression of cardiac electrical and mechanical junction proteins in response to stretch". Circulation research 97.4 (2005), pp. 346-353.

[8] H. Yang, L. P. Schmidt, Z. Wang, X. Yang, Y. Shao, T. K. Borg, R. Markwald, R. Runyan and B. Z. Gao. "Dynamic Myofibrillar Remodeling in Live Cardiomyocytes under Static Stretch". Scientific reports 6 (2016).

[9] H. E. Boycott, C. S. Barbier, C. A. Eichel, K. D. Costa, R. P. Martins, F. Louault, G. Dilanian, A. Coulombe, S. N. Hatem and E. Balse. "Shear stress triggers insertion of voltage-gated potassium channels from intracellular compartments in atrial myocytes". Proceedings of the National Academy of Sciences 110.41 (2013), E3955-E3964.

[10] M. Morad, A. Javaheri, T. Risius and S. Belmonte. "Multimodality of Ca2+ signaling in rat atrial myocytes". Annals of the New York Academy of Sciences 1047.1 (2005), pp. 112-121.

[11] I. Lorenzen-Schmidt, G. W. Schmid-Schönbein, W. R. Giles, A. D. McCulloch, S. Chien and J. H. Omens. "Chronotropic response of cultured neonatal rat ventricular myocytes to short-term fluid shear". Cell biochemistry and biophysics 46.2 (2006), pp. 113-122.

[12] V. Hariharan, A. Asimaki, J. E. Michaelson, E. Plovie, C. A. MacRae, J. E. Saffitz and H. Huang. "Arrhythmogenic right ventricular cardiomyopathy mutations alter shear response without changes in cell-cell adhesion". Cardiovascular research 104.2 (2014), pp. 280-289. 
[13] V. Y. Wang, M. P. Nash, I. LeGrice, A. A. Young, B. H. Smaill and P. J. Hunter. "Mathematical models of cardiac structure and function: mechanistic insights from models of heart failure". Cardiac Mechano-Electric Coupling and Arrhythmias (2011), pp. 241-250.

[14] A. Cheng, T. C. Nguyen, M. Malinowski, G. T. Daughters, D. C. Miller and N. B. Ingels. "Heterogeneity of left ventricular wall thickening mechanisms". Circulation 118.7 (2008), pp. 713721.

[15] A. J. Pope, G. B. Sands, B. H. Smaill and I. J. LeGrice. "Three-dimensional transmural organization of perimysial collagen in the heart". American Journal of Physiology-Heart and Circulatory Physiology 295.3 (2008), H1243-H1252.

[16] S. Rohr. "Arrhythmogenic implications of fibroblast-myocyte interactions". Circulation: Arrhythmia and Electrophysiology 5.2 (2012), pp. 442-452.

[17] I. Sarantitis, P. Papanastasopoulos, M. Manousi, N. G. Baikoussis and E. Apostolakis. "The cytoskeleton of the cardiac muscle cell". Hellenic J Cardiol 53.5 (2012), pp. 367-379.

[18] J. M. Rutkowski and M. A. Swartz. "A driving force for change: interstitial flow as a morphoregulator". Trends in cell biology 17.1 (2007), pp. 44-50.

[19] S. Göktepe, O. J. Abilez, K. K. Parker and E. Kuhl. "A multiscale model for eccentric and concentric cardiac growth through sarcomerogenesis". Journal of theoretical biology 265.3 (2010), pp. $433-442$.

[20] Ahmed Fawzy, James L.Pool Kernel Description. http://www.medscape.org/viewarticle/440787.

[21] C. A. Walker and F. G. Spinale. "The structure and function of the cardiac myocyte: a review of fundamental concepts". The Journal of thoracic and cardiovascular surgery 118.2 (1999), pp. 375382 .

[22] The Cardio Research Web Project Kernel Description. http: //www. cardio-research.com/ cardiomyocytes. Accessed: 2017-05-11.

[23] K. K. Parker. "Functional implications of myocyte architecture". Cardiac Mechano-Electric Coupling and Arrhythmias (2011), p. 87.

[24] C. Dos Remedios, D Chhabra, M Kekic, I. Dedova, M Tsubakihara, D. Berry and N. Nosworthy. "Actin binding proteins: regulation of cytoskeletal microfilaments". Physiological reviews 83.2 (2003), pp. 433-473.

[25] R. J. Solaro. "Remote control of A-band cardiac thin filaments by the IZI protein network of cardiac sarcomeres". Trends in cardiovascular medicine 15.4 (2005), pp. 148-152.

[26] C. M. Niessen. "Tight junctions/adherens junctions: basic structure and function". Journal of Investigative Dermatology 127.11 (2007), pp. 2525-2532.

[27] M. L. McCain and K. K. Parker. "Mechanotransduction: the role of mechanical stress, myocyte shape, and cytoskeletal architecture on cardiac function". Pflügers Archiv-European Journal of Physiology 462.1 (2011), p. 89.

[28] J.-C. Wu, H.-C. Sung, T.-H. Chung and R. M. DePhilip. "Role of N-cadherin-and integrin-based costameres in the development of rat cardiomyocytes". Journal of cellular biochemistry 84.4 (2002), pp. 717-724.

[29] E. Kardami and B. W. Doble. "Cardiomyocyte gap junctions: A target of growth-promoting signaling". Trends in cardiovascular medicine 8.4 (1998), pp. 180-187. 
[30] T. Desplantez. "Cardiac Cx43, Cx40 and Cx45 co-assembling: involvement of connexins epitopes in formation of hemichannels and Gap junction channels". BMC cell biology 18.1 (2017), p. 3.

[31] V. Valiunas, F. F. Bukauskas and R. Weingart. "Conductances and selective permeability of connexin43 gap junction channels examined in neonatal rat heart cells". Circulation Research 80.5 (1997), pp. 708-719.

[32] J. E. Saffitz, R. H. Hoyt, R. A. Luke, H. L. Kanter and E. C. Beyer. "Cardiac myocyte interconnections at gap junctions: role in normal and abnormal electrical conduction". Trends in cardiovascular medicine 2.2 (1992), pp. 56-60.

[33] J. D. Humphries, A. Byron and M. J. Humphries. "Integrin ligands at a glance". Journal of cell science 119.19 (2006), pp. 3901-3903.

[34] E. R. Pfeiffer, J. R. Tangney, J. H. Omens and A. D. McCulloch. "Biomechanics of cardiac electromechanical coupling and mechanoelectric feedback". Journal of biomechanical engineering 136.2 (2014), p. 021007.

[35] T. A. Quinn, R. A. Bayliss and P. Kohl. "Mechano-electric feedback in the heart: effects on heart rate and rhythm". In: Heart rate and rhythm. Springer, 2011, pp. 133-151.

[36] P. Kohl, P. Hunter and D. Noble. "Stretch-induced changes in heart rate and rhythm: clinical observations, experiments and mathematical models". Progress in biophysics and molecular biology 71.1 (1999), pp. 91-138.

[37] A. Salameh, A. Wustmann, S. Karl, K. Blanke, D. Apel, D. Rojas-Gomez, H. Franke, F. W. Mohr, J. Janousek and S. Dhein. "Cyclic mechanical stretch induces cardiomyocyte orientation and polarization of the gap junction protein connexin43". Circulation research 106.10 (2010), pp. $1592-1602$.

[38] P Kohl. "Cardiac stretch-activated channels and mechano-electric transduction". Cardiac electrophysiology: From cell to bedside (2009), pp. 115-126.

[39] M. G.S. J. Sutton and N. Sharpe. "Left ventricular remodeling after myocardial infarction". Circulation 101.25 (2000), pp. 2981-2988.

[40] J. P. Cleutjens, J. C. Kandala, E. Guarda, R. V. Guntaka and K. T. Weber. "Regulation of collagen degradation in the rat myocardium after infarction". Journal of molecular and cellular cardiology 27.6 (1995), pp. 1281-1292.

[41] G. S. Francis and K. M. McDonald. "Left ventricular hypertrophy: an initial response to myocardial injury". The American journal of cardiology 69.18 (1992), pp. 3-9.

[42] C. A. Souders, S. L. Bowers and T. A. Baudino. "Cardiac fibroblast". Circulation research 105.12 (2009), pp. 1164-1176.

[43] J Rother, C. Richter, L. Turco, F. Knoch, I Mey, S. Luther, A Janshoff, E. Bodenschatz and M. Tarantola. "Crosstalk of cardiomyocytes and fibroblasts in co-cultures". Open biology 5.6 (2015), p. 150038.

[44] B. Cooke and J Stuart. "Automated measurement of plasma viscosity by capillary viscometer." Journal of clinical pathology 41.11 (1988), pp. 1213-1216.

[45] H. M. Spotnitz, W. D. Spotnitz, T. S. Cottrell, D. Spiro and E. H. Sonnenblick. "Cellular basis for volume related wall thickness changes in the rat left ventricle". Journal of molecular and cellular cardiology 6.4 (1974), pp. 317-331. 
[46] I. Giaever and C. R. Keese. "Monitoring fibroblast behavior in tissue culture with an applied electric field". Proceedings of the National Academy of Sciences 81.12 (1984), pp. 3761-3764.

[47] I. Giaever and C. R. Keese. "Micromotion of mammalian cells measured electrically". Proceedings of the National Academy of Sciences 88.17 (1991), pp. 7896-7900.

[48] C.-M. Lo and J. Ferrier. "Impedance analysis of fibroblastic cell layers measured by electric cell-substrate impedance sensing". Physical Review E 57.6 (1998), p. 6982.

[49] S. Chaudhuri, H. Nguyen, R. M. Rangayyan, S. Walsh and C. B. Frank. "A Fourier domain directional filterng method for analysis of collagen alignment in ligaments". IEEE transactions on biomedical engineering 7 (1987), pp. 509-518.

[50] F. J. Harris. "On the use of windows for harmonic analysis with the discrete Fourier transform". Proceedings of the IEEE 66.1 (1978), pp. 51-83.

[51] S. G. Shroff, D. R. Saner and R. Lal. "Dynamic micromechanical properties of cultured rat atrial myocytes measured by atomic force microscopy". American Journal of Physiology-Cell Physiology 269.1 (1995), pp. C286-C292.

[52] J. Alcaraz, L. Buscemi, M. Grabulosa, X. Trepat, B. Fabry, R. Farré and D. Navajas. "Microrheology of human lung epithelial cells measured by atomic force microscopy". Biophysical journal 84.3 (2003), pp. 2071-2079.

[53] G. Bilodeau. "Regular pyramid punch problem". J. Appl. Mech 59.3 (1992), pp. 519-523.

[54] J Alcaraz, L Buscemi, M Puig-de Morales, J Colchero, A Baro and D Navajas. "Correction of microrheological measurements of soft samples with atomic force microscopy for the hydrodynamic drag on the cantilever". Langmuir 18.3 (2002), pp. 716-721.

[55] J. Rother, H. Nöding, I. Mey and A. Janshoff. "Atomic force microscopy-based microrheology reveals significant differences in the viscoelastic response between malign and benign cell lines". Open biology 4.5 (2014), p. 140046.

[56] L. Limozin and K. Sengupta. "Quantitative reflection interference contrast microscopy (RICM) in soft matter and cell adhesion". ChemPhysChem 10.16 (2009), pp. 2752-2768.

[57] J. Baum and H. S. Duffy. "Fibroblasts and myofibroblasts: what are we talking about?" Journal of cardiovascular pharmacology 57.4 (2011), p. 376.

[58] F. Klingberg, B. Hinz and E. S. White. "The myofibroblast matrix: implications for tissue repair and fibrosis". The Journal of pathology 229.2 (2013), pp. 298-309.

[59] Y. Zhang, E. M. Kanter and K. A. Yamada. "Remodeling of cardiac fibroblasts following myocardial infarction results in increased gap junction intercellular communication". Cardiovascular Pathology 19.6 (2010), e233-e240.

[60] D. M. Pedrotty, R. Y. Klinger, N. Badie, S. Hinds, A. Kardashian and N. Bursac. "Structural coupling of cardiomyocytes and noncardiomyocytes: quantitative comparisons using a novel micropatterned cell pair assay". American Journal of Physiology-Heart and Circulatory Physiology 295.1 (2008), H390-H400.

[61] Y. Asazuma-Nakamura, P. Dai, Y. Harada, Y. Jiang, K. Hamaoka and T. Takamatsu. "Cx43 contributes to TGF- $\beta$ signaling to regulate differentiation of cardiac fibroblasts into myofibroblasts". Experimental cell research 315.7 (2009), pp. 1190-1199. 
[62] E. Ongstad and P. Kohl. "Fibroblast-myocyte coupling in the heart: potential relevance for therapeutic interventions". Journal of molecular and cellular cardiology 91 (2016), pp. 238-246.

[63] S. A. Thompson, C. R. Copeland, D. H. Reich and L. Tung. "Mechanical Coupling Between Myofibroblasts and Cardiomyocytes Slows Electric Conduction in Fibrotic Cell MonolayersClinical Perspective". Circulation 123.19 (2011), pp. 2083-2093.

[64] T. P. Nguyen, Y. Xie, A. Garfinkel, Z. Qu and J. N. Weiss. "Arrhythmogenic consequences of myofibroblast-myocyte coupling". Cardiovascular research 93.2 (2012), pp. 242-251.

[65] C. Vasquez, P. Mohandas, K. L. Louie, N. Benamer, A. C. Bapat and G. E. Morley. "Enhanced Fibroblast-Myocyte Interactions in Response to Cardiac InjuryNovelty and Significance". Circulation research 107.8 (2010), pp. 1011-1020.

[66] G. Gaudesius, M. Miragoli, S. P. Thomas and S. Rohr. "Coupling of cardiac electrical activity over extended distances by fibroblasts of cardiac origin". Circulation research 93.5 (2003), pp. 421428.

[67] H.-H. Gerdes and R. N. Carvalho. "Intercellular transfer mediated by tunneling nanotubes". Current opinion in cell biology 20.4 (2008), pp. 470-475.

[68] S. A. Thompson, A. Blazeski, C. R. Copeland, D. M. Cohen, C. S. Chen, D. M. Reich and L. Tung. "Acute slowing of cardiac conduction in response to myofibroblast coupling to cardiomyocytes through N-cadherin". Journal of molecular and cellular cardiology 68 (2014), pp. 29-37.

[69] C. Rosker, N. Salvarani, S. Schmutz, T. Grand and S. Rohr. "Abolishing myofibroblast arrhythmogeneicity by pharmacological ablation of $\alpha$-smooth muscle actin containing stress fibers". Circulation research (2011), CIRCRESAHA-111.

[70] J. Pellman, J. Zhang and F. Sheikh. "Myocyte-fibroblast communication in cardiac fibrosis and arrhythmias: mechanisms and model systems". Journal of molecular and cellular cardiology 94 (2016), pp. 22-31.

[71] B. Fabry, G. N. Maksym, J. P. Butler, M. Glogauer, D. Navajas and J. J. Fredberg. "Scaling the microrheology of living cells". Physical review letters 87.14 (2001), p. 148102.

[72] M. M. Zegers and P. Friedl. "Translating Membrane Tension into Cytoskeletal Action by FBP17". Developmental cell 33.6 (2015), pp. 628-630.

[73] P. DiMilla, K. Barbee and D. Lauffenburger. "Mathematical model for the effects of adhesion and mechanics on cell migration speed". Biophysical journal 60.1 (1991), pp. 15-37.

[74] A. Gefen. Cellular and biomolecular mechanics and mechanobiology. Springer, 2011.

[75] D. Schneider, T. Baronsky, A. Pietuch, J. Rother, M. Oelkers, D. Fichtner, D. Wedlich and A. Janshoff. "Tension monitoring during epithelial-to-mesenchymal transition links the switch of phenotype to expression of moesin and cadherins in NMuMG cells". PLoS One 8.12 (2013), e80068.

[76] L. B. Katsnelson, T. Sulman, O. Solovyova and V. S. Markhasin. "Role of myocardial viscoelasticity in disturbances of electrical and mechanical activity in calcium overloaded cardiomyocytes: mathematical modeling". Journal of theoretical biology 272.1 (2011), pp. 83-95.

[77] J. Michaelson, V. Hariharan and H. Huang. "Hyperglycemic and hyperlipidemic conditions alter cardiac cell biomechanical properties". Biophysical journal 106.11 (2014), pp. 2322-2329. 
[78] G. K. Ragsdale, J. Phelps and K. Luby-Phelps. "Viscoelastic response of fibroblasts to tension transmitted through adherens junctions". Biophysical journal 73.5 (1997), pp. 2798-2808.

[79] P. F. Davies, A. Robotewskyj and M. L. Griem. "Quantitative studies of endothelial cell adhesion. Directional remodeling of focal adhesion sites in response to flow forces." Journal of Clinical Investigation 93.5 (1994), p. 2031.

[80] N. DePaola, J. E. Phelps, L. Florez, C. R. Keese, F. L. Minnear, I. Giaever and P. Vincent. "Electrical impedance of cultured endothelium under fluid flow". Annals of biomedical engineering 29.8 (2001), pp. 648-656.

[81] S. Y. Ng, C. K. Wong and S. Y. Tsang. "Differential gene expressions in atrial and ventricular myocytes: insights into the road of applying embryonic stem cell-derived cardiomyocytes for future therapies". American Journal of Physiology-Cell Physiology 299.6 (2010), pp. C1234-C1249.

[82] S. Nishimura, S. Nagai, M. Katoh, H. Yamashita, Y. Saeki, J.-i. Okada, T. Hisada, R. Nagai and S. Sugiura. "Microtubules modulate the stiffness of cardiomyocytes against shear stress". Circulation Research 98.1 (2006), pp. 81-87.

[83] S. Hein, S. Kostin, A. Heling, Y. Maeno and J. Schaper. "The role of the cytoskeleton in heart failure". Cardiovascular research 45.2 (2000), pp. 273-278.

[84] C. P. Ng, B. Hinz and M. A. Swartz. "Interstitial fluid flow induces myofibroblast differentiation and collagen alignment in vitro". Journal of cell science 118.20 (2005), pp. 4731-4739.

[85] C.-M. Lo, C. R. Keese and I. Giaever. "Impedance analysis of MDCK cells measured by electric cell-substrate impedance sensing". Biophysical journal 69.6 (1995), pp. 2800-2807.

[86] T. Dvir, O. Levy, M. Shachar, Y. Granot and S. Cohen. "Activation of the ERK1/2 cascade via pulsatile interstitial fluid flow promotes cardiac tissue assembly". Tissue engineering 13.9 (2007), pp. 2185-2193.

[87] M. Sato, M. J. Levesque and R. M. Nerem. "Micropipette aspiration of cultured bovine aortic endothelial cells exposed to shear stress." Arteriosclerosis, Thrombosis, and Vascular Biology 7.3 (1987), pp. 276-286.

[88] A. Van der Meer, A. Poot, J Feijen and I Vermes. "Analyzing shear stress-induced alignment of actin filaments in endothelial cells with a microfluidic assay". Biomicrofluidics 4.1 (2010), p. 011103.

[89] S. Li, P. Butler, Y. Wang, Y. Hu, D. C. Han, S. Usami, J.-L. Guan and S. Chien. "The role of the dynamics of focal adhesion kinase in the mechanotaxis of endothelial cells". Proceedings of the National Academy of Sciences 99.6 (2002), pp. 3546-3551.

[90] J. M. Archambault, M. K. Elfervig-Wall, M. Tsuzaki, W. Herzog and A. J. Banes. "Rabbit tendon cells produce MMP-3 in response to fluid flow without significant calcium transients". Journal of biomechanics 35.3 (2002), pp. 303-309.

[91] K. K. Parker, J. Tan, C. S. Chen and L. Tung. "Myofibrillar architecture in engineered cardiac myocytes". Circulation research 103.4 (2008), pp. 340-342.

[92] N. A. Geisse, S. P. Sheehy and K. K. Parker. "Control of myocyte remodeling in vitro with engineered substrates". In Vitro Cellular $\&$ Developmental Biology-Animal 45.7 (2009), pp. 343350 . 
[93] Y. G. Wang, A. M. Samarel and S. L. Lipsius. "Laminin acts via $\beta 1$ integrin signalling to alter cholinergic regulation of L-type Ca2+ current in cat atrial myocytes". The Journal of physiology 526.1 (2000), pp. 57-68.

[94] T. A. Quinn, P. Camelliti, U. Siedlecka, T. Poggioli, L. M. Loew, T. Knöpfel and P. Kohl. "Cell-specific expression of voltage-sensitive protein confirms cardiac myocyte to non-myocyte electrotonic coupling in healed murine infarct border tissue". Circulation 130.Suppl 2 (2014), A11749-A11749. 



\section{Acknowledgments}

This thesis would have not been possible without the tremendous scientific and personal support and motivation of Dr. Isabella Guido, who has taught me, both consciously and un-consciously, how a good scientist should be, and Dr. Narain Karedla. They have been essential for their invaluable advices and feedback on my research. They were always ready to solve experimental and personal problems through the years. I also thank them for their patience and time going through this thesis line by line.

I would like to thank Dr. Marco Tarantola for supervising my thesis and providing the starting idea of this project.

I thank the members of my thesis committee, Prof. Stephan Luther and Prof. Claudia Steinem for the fruitful suggestions whenever I required, and taking their precious time out to regularly monitor and discuss the progress of my work.

I am grateful to Prof. Dr. Bodenschatz for the fruitful discussions concerning my work and the support of my scientific activity in his department.

My sincere thanks goes to Azam, Claudia, Filippo and Albert for their technical support and suggestions related to my work, and also for reading parts of my thesis.

I am grateful to all the technical staff from the workshop and the lab, especially Marion, Tina and Alex who isolated cells for my experiments.

I acknowledge the financial support from the Niedersächsisches Ministerium für Wissenschaft und Kultur for providing the IMPRS Excellence Stipend.

Many thanks to the complete GGNB team for their tireless guidance through all the formalities.

Very special thanks go to my adopted family including Marcin, Marco, Oliver, Julien, Arnaud, Hakam, Isabella, Narain and Cristina for the good time we had together and for being there to listen whenever I needed an ear.

I thanks my office mates, my colleagues and friends especially Nadine, Kaumudi and Smrithika for all the stories, jokes, dinners over these years.

Lastly, I would like to thanks my family for all their encouragement and support. 



\section{Glossary}

MI Myocardial Infarction

NRVMS Neonatal Rat Ventricular Myocytes

Cx43 Connexin 43

N-Cad Neural-Cadherin

ECM Extra Cellular Matrix

ATP Adenosine TriPhosphate

SR Sarcoplasmic Reticulum

SERCA Sarco Endoplasmic Reticulum $\mathrm{Ca}^{2+}$-ATPase

MEC Mechano-Electric Coupling

TGF- $\beta 1$ Tumor Growth Factor- $\beta 1$

$\alpha$-SMA $\quad \alpha$-Smooth Muscle Actin

CM Cardiomyocytes

$\mathrm{Fb} \quad$ Fibroblasts

PBS Phosphate Buffered Saline

EDTA EthyleneDiamineTetraetic Acid)

DMSO DyMethylSulfOxide

FCS Fetal Calf Serum

DMEM Dulbeccos's Modified Eagle's Medium

ECIS Electric Cell-substrate Impedance Spectroscopy

AFM Atomic Force Microscopy

RICM Reflection Interference Constrast Microscopy

PFA ParaFormAldehyle

BSA Bovine Serum Albumin

DNA DeoxyriboNucleic Acids

DAPI 4,6 DiAmidino-2-PhenyIndole

CPE Constant Phase Element

RTC Rapid Time Collect

SFT Single Frequency Time 
Glossary

MFT Multi Frequency Time

FFT Fast Fourier Transformation

PSD Power Spectral Density

CLSM Confocal Laser Scanning Microscopy

FT Fourier Transform

QD Quadrant Photodiode

PIPC Propagation Induced Phase Constrast

ERK Extracellular signal Regulated Kinases 


\section{Curriculum Vitae}

Turco Laura

Graduate in Biomedical Engineering (M. Sc. Tissue Engineering) born, October $4^{\text {rd }} 1988$ in Vizzolo P. (MI), Italy

\section{Academical Background}

\begin{tabular}{l|l}
$10.2013-$ present & $\begin{array}{l}\text { Doctoral Dissertation } \\
\text { Max Planck Institute for Dynamics and Self-Organization } \\
\text { Götingen, Germany } \\
\text { Dr. Marco Tarantola }\end{array}$ \\
$09.2010-04.2013$ & $\begin{array}{l}\text { Master of Science } \\
\text { Biomedical Engineering } \\
\text { Politecnico di Milano, Italy }\end{array}$ \\
$03.2012-04.2013$ & $\begin{array}{l}\text { Master Thesis } \\
\text { Development of a micro-bioreactor for uniaxial mechanical stimulation } \\
\text { of monolayer cell culture } \\
\text { Laboratory of Micro- and Bio-Fluid Dynamics } \\
\text { Politecnico di Milano, Italy } \\
\text { Prof. Dr. Gianfranco Beniamino Fiore }\end{array}$ \\
$01.2011-06.2011$ & $\begin{array}{l}\text { Erasmus Research Program } \\
\text { Uppsala Universitet, Sweden }\end{array}$ \\
Research Internship \\
Mechanical evaluation of an injectable poly (trimethylenecarbonate)
\end{tabular} \mid




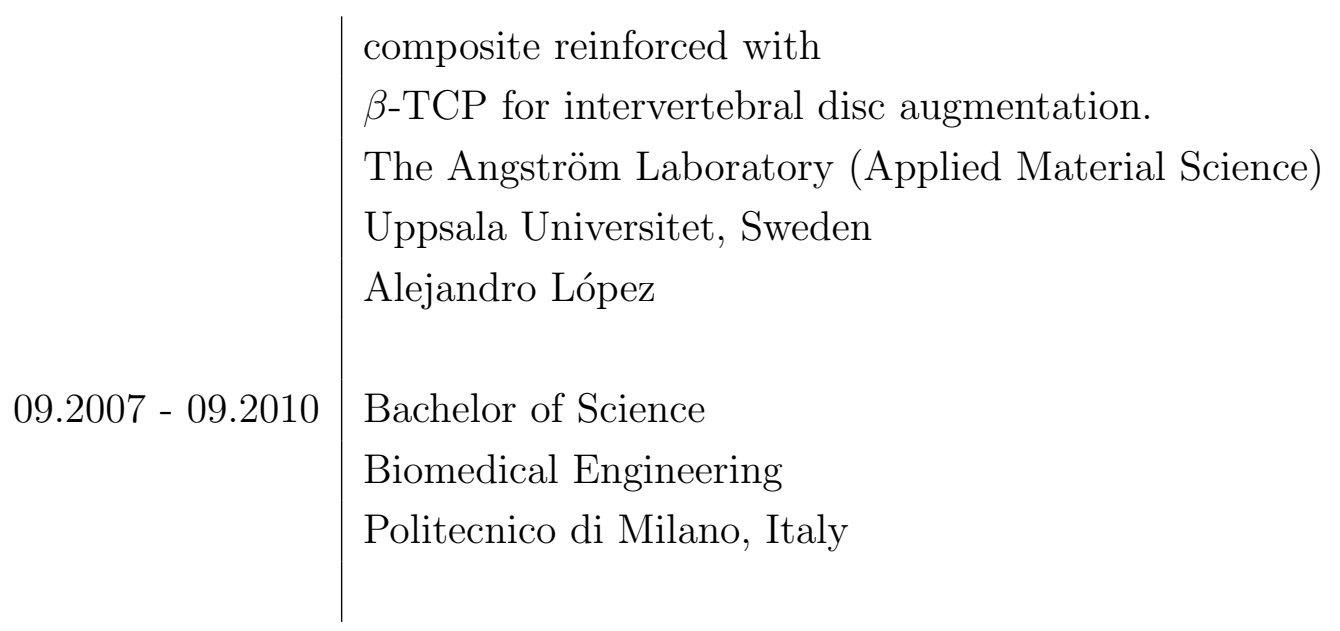

Talks \& Posters

03.2017

09.2016

02.1016

American Physical Society March Meeting 2017

New Orleans, LA, USA

"Dynamics of Cardiac Laminar Sheets under Shear Stress"

7th International Workshop

Cardiac Mechano-Electric Coupling and Arrhythmias, Freiburg, Germany

"Dynamics and mechanics of cardiac fibrosis"

Kyoto Winter School 2016

From Materials to Life: Multidisciplinary Challenges

Kyoto, Japan

"Dynamics and mechanics of cardiac fibrosis"

03.2015

Keystone Symposia Conference

Copper Mountain, Colorado, USA

"Electromechanical coupling of cardiomyocytes under fibrotic conditions

Fellowships, Awards and Achievements

04.2015 - 04.2017 Ministerium für Wissenschaft und Kultur (MWK) excellence stipend Lower Saxony, Germany 


\section{Other Professional Activities}

2016 - date

2016 - date

$02.2015-02.2016$

and Self-Organization

2016

2016
MPI-DS and IMPRS career seminars organizer

$3^{\text {rd }}$ Third Infinity conference organizer

Student representative of Max Planck for Dynamics

$2^{\text {nd }}$ Third Infinity conference organizer

$2^{\text {nd }}$ Graduate school retreat organizer

\section{Publications}

Turco, L.; Karedla N.; Tarantola, M.

"Effect of shear stress in cardiac laminar sheets"

in preparation

Kleisch, T. T., Deitz, J.; Turco, L.; Halder, P.; Polo, E.; Tarantola, M.; Jahn, R.;

Janshoff, A. "Membrane tension increases fusion efficiency of model membranes $n$ the presence of SNAREs" Scientific Reports under revision

Rother, J.; Richter, C.; Turco, L.; Knoch, F.; Mey, I.; Luther, S.; Janshoff, A.;

Bodenschatz, E.; Tarantola, M. "Crosstalk of cardiomycytes and

fibroblasts in co-cultures." Open Biology 5150038 (2015) 